\title{
Métodos de Diagnóstico em Modelos Autoregressivos Simétricos
}

\author{
Márcio José de Medeiros \\ DisSERTAÇÃO APRESENTADA AO \\ Instituto de Matemática e Estatística \\ da Universidade de São Paulo Como \\ REQUiSito PARA OBTENÇÃo DO TítULO DE \\ Mestre em Estatística \\ Área de Concentração: Estatística \\ Orientador: Prof. Dr. Gilberto Alvarenga Paula
}

São Paulo, novembro de 2006 


\title{
Validação de Modelos Autoregressivos Simétricos
}

\author{
Este exemplar corresponde à redação \\ final da dissertação devidamente corrigida e \\ defendida por Márcio José de Medeiros \\ e aprovada pela comissão julgadora.
}

São Paulo, 17 de novembro de 2006.

Comissão julgadora:

- Prof. Dr. Gilberto Alvarenga Paula - IME/USP

- Prof. Dra. Viviana Giampaoli - IME/USP

- Prof. Dr. Filidor Edifonso Vilca Labra - IMECC/UNICAMP 
"Num relógio

É quatro e vinte

No outro é quatro e meia

É que de um relógio pra outro As horas vareia" 
"És um poema na terra Uma estrela no céu, um tesouro no mar És tanta felicidade Que nem consigo exaltar" 


\section{Agradecimentos}

Em primeiro lugar, ao professor Gilberto Paula, pela orientação, pelas diversas oportunidades que me ofereceu e, principalmente, pelo estímulo sempre demonstrado. Sem sua atenção, este trabalho não teria sido concluído.

Aos professores do DEMA/UFC - Departamento de Estatística e Matemática Aplicada da Universidade Federal do Ceará - por todo o incentivo dispensado durante a minha graduação, pela motivação para eu continuar a estudar, pela amizade e convívio. Em especial aos professores: Ana Araújo, João Alexandre, João Mota, Manoel Campêlo, Rosa Mota e Silvia Freitas.

À Claudia Mont' Alverne, da Pró-Reitoria de Assistência Estudantil da Universidade Federal do Ceará, pelo apoio e disponibilidade.

Aos meus amigos, pelo apoio constante, por fazerem parte da minha vida. Especialmente a: Cleudimar Dantas, Elizete Américo, Eugênia Alencar, Jony Arrais, José dos Santos e Patrícia Viana.

Ao amigo Marcelo Pinheiro, em quem me espelho por suas qualidades humanas e éticas.

Aos colegas do IME/USP com os quais tive o privilégio de conviver e aprender. Em especial à Michelli Silva, por oferecer críticas 
e sugestões extremamente valiosas a este trabalho, por seu exemplo de determinação e busca pela perfeição.

Agradecimento especial ao amigo Carlos Fagundes, com quem tive o privilégio de aprender e trocar idéias, desde que nos conhecemos no Mestrado Profissionalizante em Modelagem Matemática em Finanças.

À toda minha família, guia da minha jornada e alicerce do que sou, exprimo o meu profundo agradecimento. 


\section{Resumo}

Os modelos autoregressivos simétricos são modelos de regressão em que os erros são correlacionados e pertencem à classe de distribuições simétricas. Normalmente, dados observados ao longo do tempo (em seqüência) apresentam alguma correlação. Exemplos desse tipo de dados são encontrados freqüentemente em economia. Em muitas dessas aplicações, espera-se que a autocorrelação decresça fortemente com a "distância" entre as observações e, por isso, adota-se a estrutura AR(1) para os erros. Não é usual nesse tipo de modelo deletar dados para avaliar o impacto das observações nas estimativas dos parâmetros, característica usual dos métodos de diagnóstico para observações independentes. O objetivo deste trabalho é discutir métodos de diagnóstico de influência para esses modelos. Para ilustrar a metodologia, são apresentados exemplos do modelo de precificação de ativos (CAPM). 


\section{Abstract}

The symmetric autoregressive models are regression models in which the errors are correlated and belong to the class of symmetrical distributions. Usually, data observed along the time (in sequence) present some correlation. Examples of this kind of data are frequently found in economy. In many of these applications, the autocorrelation is expected to decrease strongly with the "distance" between observations and, for this reason, the structure $\operatorname{AR}(1)$ is adopted for the errors. It is notorial in this kind of model to delete data for evaluating the impact of the observations on the parameter estimator, a characteristic of the diagnotic methods for independent observations. The aim of this work is to discuss influence diagnostic methods for those models. To illustrate the methodology, examples of Capital Asset Pricing Models (CAPM) are presented. 


\section{Sumário}

1 Introdução 1

1.1 Apresentação do problema e definição dos objetivos . . 1

1.2 Apresentação dos capítulos . . . . . . . . . . . . . . 4

1.3 Distribuições elípticas . . . . . . . . . . . . . . . . . . . 4

1.3 .1 Propriedades . . . . . . . . . . . . . . 6

1.3.2 Alguns exemplos de distribuições elípticas . . . 8

2 Modelos simétricos de regressão linear 14

2.1 Definição do modelo . . . . . . . . . . . . . . . 15

2.2 Função escore e informação de Fisher . . . . . . . . . 16

2.2 .1 Função escore . . . . . . . . . . . . . . . . . . . . 16

2.2 .2 Informação de Fisher . . . . . . . . . . . . . . 18 
2.3 Estimação . . . . . . . . . . . . . . . . . . . . . . . . 20

2.4 Exemplo . . . . . . . . . . . . . . . . . . . . . 21

2.4.1 Modelo CAPM das ações da Telemar . . . . . . 21

3 Modelos simétricos de regressão linear com erros AR(1) 29

3.1 Definição do modelo . . . . . . . . . . . . . . . . . . . 31

3.2 Função escore e informação de Fisher . . . . . . . . . . 33

3.2 .1 Função escore . . . . . . . . . . . . . . . . . . . . . 33

3.2 .2 Informação de Fisher . . . . . . . . . . . . . . 36

3.3 Estimação . . . . . . . . . . . . . . . . . . . . . . . . . . . . 39

3.4 Exemplo . . . . . . . . . . . . . . . . . . . . . . 40

3.4 .1 Modelo CAPM . . . . . . . . . . . . . . . . . 40

4 Métodos de diagnóstico em modelos simétricos de regressão linear com erros AR(1)

4.1 Resíduos . . . . . . . . . . . . . . . . . . . . . . . 47

4.2 Pontos de alavanca . . . . . . . . . . . . . . . . . . . . . 49

4.3 Influência local . . . . . . . . . . . . . . . . . . . 51

4.3.1 Perturbação aditiva na resposta dos modelos simétricos de regressão com erros $\mathrm{AR}(1) \ldots \ldots$ 
4.3.2 Perturbação aditiva na $i$-ésima variável explicativa dos modelos simétricos de regressão com er-

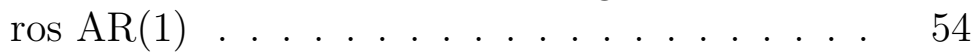

4.4 Exemplos . . . . . . . . . . . . . . . 55

4.4.1 Continuação do Exemplo 2 . . . . . . . . . . 55

5 Conclusões

$\begin{array}{ll}\text { A Vetor gradiente } & 67\end{array}$

$\begin{array}{ll}\text { B Matriz hessiana } & 70\end{array}$

$\begin{array}{ll}\text { C Pontos de alavanca } & 76\end{array}$

$\begin{array}{lr}\text { D Influência local } & 80\end{array}$ 


\section{Lista de Figuras}

1.1 Gráficos das funções de densidade marginal e bivariada da distribuição normal padrão. . . . . . . . . . . . . . 9

1.2 Gráficos de funções de densidade marginal e bivariada da distribuição t-Student. . . . . . . . . . . . . 10

1.3 Gráficos das funções de densidade marginal e bivariada da distribuição de Laplace. . . . . . . . . . . . . . . . . 11

1.4 Gráficos de funções de densidade marginal e bivariada da distribuição Pearson VII. . . . . . . . . . . . . . . . 12

1.5 Gráficos das distribuições normal padrão, $t$-Student com $\nu=3$, Laplace e Pearson tipo VII com $N=1$ e $m=10$.

1.6 Curvas de nível das distribuições normal padrão, $t$-Student com $\nu=3$, Laplace e Pearson tipo VII com $N=1 \mathrm{e}$ $m=10 \ldots \ldots \ldots \ldots \ldots$

2.1 Retornos mensais da Telemar e do Ibovespa. . . . . . 
2.2 Reta ajustada, supondo distribuição normal para os erros, e gráfico de dispersão entre os excessos de retorno do Ibovespa e Telemar. . . . . . . . . . . . . . . . . .

2.3 Reta ajustada, supondo distribuição t-Student com 3 g.l. para os erros, e gráfico de dispersão entre os excessos de retorno do Ibovespa e Telemar. . . . . . . . . . . .

2.4 Retas ajustadas de acordo com as suposições para os erros e gráfico de dispersão entre os excessos de retorno do Ibovespa e Telemar. . . . . . . . . . . . . . . . .

3.1 Retornos mensais do Itaú e do Ibovespa. . . . . . . . .

3.2 Reta ajustada pelos modelos de regressão autoregressivo e normal linear e gráfico de dispersão entre os excessos de retorno do Ibovespa e Itaú. . . . . . . . . . . . . . .

4.1 Reta ajustada e diagrama de dispersão entre os excessos de retorno do Ibovespa e do Itaú. . . . . . . . . . . . 56

4.2 (a) Resíduos, (b) função de autocorrelação e (c) função de autocorrelação parcial dos resíduos para o ajuste apresentado pela Tabela 4.1. . . . . . . . . . . . . .

4.3 (a) Resíduos, (b) função de autocorrelação e (c) função de autocorrelação parcial dos resíduos definidos por (4.5) para o ajuste apresentado pela Tabela 4.1. . . . . . 58

4.4 (a) Percentis da distribuição normal versus percentis dos resíduos padronizados e (b) histograma dos resíduos padronizados. . . . . . . . . . . . . . 59

4.5 Medida de alavanca - Distribuição normal. . . . . . . . 60 
4.6 Medidas de influência (distribuição normal): (a) influência da variável resposta (Excesso Itaú) nas estimativas de $\beta$, (b) dos parâmetros $\phi$ e $\rho$,(c) influência da variável explicativa (Excesso Ibovespa) nas estimativas de $\beta$, (d)

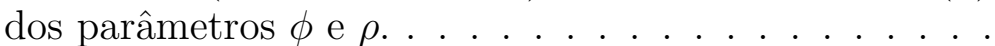

4.7 Reta ajustada e diagrama de dispersão entre os excessos de retorno do Ibovespa e do Itaú. . . . . . . . . . . . .

4.8 Medidas de influência (distribuição t-Student $\operatorname{com} \nu=$ 5): (a) influência da variável resposta (Excesso Itaú) nas estimativas de $\beta$, (b) dos parâmetros $\phi$ e $\rho$,(c) influência da variável explicativa (Excesso Ibovespa) nas estimativas de $\beta,(\mathrm{d})$ dos parâmetros $\phi$ e $\rho$. . . . . . . . . 


\section{Lista de Tabelas}

2.1 Expressões para $W_{g}(u)$ e $W_{g}^{\prime}(u)$ para algumas distribuições simétricas. . . . . . . . . . . . . 17

2.2 Valores de $d_{g}, f_{g}$ e $\xi$ para algumas distribuições simétricas. 19

2.3 Estimativas dos parâmetros do modelo CAPM supondo distribuição normal para os erros. . . . . . . . . . . . . . 24

2.4 Estimativas dos parâmetros do modelo CAPM supondo distribuição $t$-Student com 3 graus de liberdade para os erros. . . . . . . . . . . . . . . . 27

3.1 Expressões para $W_{g}(u)$ e $W_{g}^{\prime}(u)$ para algumas distribuições elípticas. . . . . . . . . . . . . . 35

3.2 Valores de $d_{g}$ e $f_{g}$ para algumas distribuições elípticas. $\quad 38$

3.3 Estimativas dos parâmetros do modelo (3.36) - (3.37) para o Banco Itaú, supondo distribuição normal para os choques aleatórios $\delta$ 's. . . . . . . . . . . . . . 
3.4 Estimativas dos parâmetros do modelo (3.36) - (3.37) para o Banco Itaú, supondo distribuição normal para os choques aleatórios $\delta$ 's. ..............

3.5 Estimativas dos parâmetros do modelo CAPM para o Banco Itaú, supondo distribuição normal com erros independentes. . . . . . . . . . . . . . . .

4.1 Estimativas dos parâmetros do modelo (3.36) - (3.37) para o Banco Itaú, supondo distribuição normal para os choques aleatórios $\delta$ 's. . . . . . . . . . . . .

4.2 Estimativas dos parâmetros do modelo (3.36) - (3.37) para o Banco Itaú, supondo distribuição $t$-Student para os choques aleatórios $\delta$ 's. . . . . . . . . . . . 
Capítulo 1

\section{Introdução}

\subsection{Apresentação do problema e definição dos objetivos}

Análise de regressão é uma metodologia estatística para explorar o relacionamento entre duas ou mais variáveis. Em particular, regressão linear explora os relacionamentos que são descritos por retas, ou sua generalização para qualquer dimensão. Um número muito grande de problemas em praticamente todas as áreas do conhecimento pode ser analisado usando técnicas de regressão linear. Usualmente, supõese que os erros têm distribuição normal e são não-correlacionados, obtendo-se simplificações e propriedades convenientes.

Durante muitos anos os modelos normais lineares foram utilizados para descrever a maioria dos fenômenos aleatórios. Mesmo quando os fenômenos em estudo não apresentavam uma resposta para a qual fosse razoável a suposição de normalidade, tentava-se algum tipo de transformação no sentido de se alcançar a normalidade procurada (vide, por exemplo, Paula, 2004). No entanto, de modo geral, trans- 
formações apresentam desvantagens, como por exemplo, os parâmetros podem não ser facilmente interpretáveis. Além disso, as estimativas de mínimos quadrados obtidas para os coeficientes do modelo de regressão normal linear são sensíveis a observações extremas. Nas últimas décadas essas técnicas foram estendidas através do desenvolvimento de métodos robustos e generalizações do modelo de regressão normal linear (vide, por exemplo, Lange, Little e Taylor, 1989). Um exemplo são os modelos lineares generalizados (vide McCullagh e Nelder, 1989), que evitam transformações na resposta.

Além da sensibilidade às observações extremas, constatou-se que em muitas aplicações a suposição de erros não correlacionados pode não ser satisfeita. Relevantemente, esse problema pode ocorrer em dados de séries temporais. Como as observações são dispostas em ordem cronológica, é provável que existam correlações entre as mesmas. Quando os erros têm autocorrelação positiva os seguintes problemas podem ocorrer para as estimativas obtidas pelo método de mínimos quadrados (vide Montgomery et al., 2001):

- Os coeficientes estimados são não viesados, mas não têm variância mínima e, portanto, podem ser ineficientes;

- A variância pode ser subestimada e como conseqüência o erro padrão das estimativas dos coeficientes da regressão é subestimado. Assim, os intervalos de confiança serão menores do que realmente são, e os testes de hipóteses para os coeficientes individuais da regressão podem indicar que uma ou mais variáveis regressoras contribuam significativamente para o modelo quando elas realmente não contribuem. Genericamente, subestimar $\sigma^{2}$ dá ao analista a falsa impressão de acurácia;

- Os intervalos de confiança e testes de hipóteses baseados nas distribuições $t$ e $F$ não são apropriados.

Ao se considerar um modelo de regressão, muitas vezes não há certeza de que o mesmo é apropriado; os dados podem não satisfazer a uma ou mais das suposições assumidas, como por exemplo: a linearidade da função de regressão ou a independência dos erros. Portanto, para se fazer inferências é imprescindível avaliar se o modelo é 
adequado aos dados. Esta avaliação pode ser feita através de técnicas simples, como gráficos baseados nos resíduos para detectar a presença de outliers, ou técnicas (medidas) mais sofisticadas, como a Distância de Cook (Cook, 1977).

Uma técnica proposta por Cook (1986), que pode ser usada para o caso de séries temporais, é a influência local, que avalia pequenas perturbações no modelo. Tais perturbações devem ser definidas de modo conveniente, para permitir uma interpretação dos resultados obtidos. Uma das vantagens da influência local é que não se faz necessário excluir observações no processo de ajuste do modelo, permitindo avaliar o quanto uma observação influencia neste processo de modo conjunto. Esta é a principal diferença entre influência local e as demais medidas, que são baseadas em deleção. No caso correlacionado, excluir uma observação pode descaracterizar a estrutura de correlação, portanto, não faz sentido excluir uma observação para avaliar o impacto dela no modelo, tornando a técnica de influência local mais apropriada.

O problema das observações extremas pode ser contornado assumindo-se para os erros distribuições com caudas mais pesadas do que a normal, reduzindo-se a influência destes pontos. As distribuições elípticas podem ser usadas neste caso (vide, por exemplo, Cysneiros, 2004). Quanto ao problema dos erros correlacionados, pode-se supor que os erros se comportem de acordo com um modelo ARMA. Em particular, o modelo $\mathrm{AR}(1)$ tem sido usado em muitas aplicações com resultados satisfatórios.

O objetivo deste trabalho é propor um modelos de regressão com erros pertencentes à classe elíptica e com estrutura autoregressiva de ordem um - AR(1) - e discutir técnicas de diagnóstico para esse modelo. Contornando, portanto, problemas relacionados à presença de observações extremas e adotando-se uma metodologia adequada quando a suposição de independência não é satisfeita. As técnicas empregadas não estão disponíveis na literatura para esse caso particular. 


\subsection{Apresentação dos capítulos}

O Capítulo 2 trata os modelos simétricos de regressão linear e como aplicação das técnicas desenvolvidas estima-se o risco sistemático de um ativo através de um modelo da classe CAPM (Capital Asset Pricing Models), regressão usual para administradores financeiros, que trata de retornos de portfólios. Ajusta-se o CAPM supondo que os erros têm distribuição normal, suposição usual, e que têm distribuição $t$-Student, comparando-se os resultados.

O Capítulo 3 propõe os modelos simétricos de regressão com erros AR(1). Esse capítulo apresenta a modelagem estatística para essa estrutura de erro. A metodologia apresentada é usada para estimar os parâmetros do modelo CAPM, que tem como motivação o fato do modelo relacionar linearmente séries temporais, ou seja, é razoável admitir que se possa encontrar autocorrelação nos erros.

No Capítulo 4 são apresentadas técnicas de diagnóstico apropriadas aos modelos simétricos de regressão com erros AR(1). Em especial, duas perturbações são definidas. Essas perturbações podem ser estendidas a qualquer modelo autocorrelacionado. Além das técnicas, são apresentados estudos de diagnóstico para os exemplos dos Capítulos 2 e 3.

O Capítulo 5 traz resumidamente as conclusões deste trabalho.

\section{$1.3 \quad$ Distribuições elípticas}

Esta seção introduz a classe de distribuições elípticas, bem como algumas de suas propriedades.

A classe elíptica preserva a estrutura simétrica (simetria elíptica) das distribuições normais (vide, por exemplo, Arellano-Valle, 1994) e, portanto, pode ser vista como uma extensão natural das distribuições normais (vide Fang, Kotz e Ng; 1990). As distribuições elípticas têm 
se tornado popular em ciências atuariais, seguros e finanças por permitirem grande flexibilidade na modelagem das caudas ou extremos, problemas freqüentes nessas áreas. Como exemplo de aplicação, pode-se citar a análise de média-variância de retornos com distribuição elíptica discutida por Engels (2004).

Os resultados que se seguem fornecem as bases para a maioria das aplicações das distribuições elípticas. Para maiores detalhes destes resultados sugere-se consultar Fang, Kotz e Ng (1990) e Cambanis, Huang e Simons (1981).

Definição 1.1. O vetor aleatório $\mathbf{y}=\left(y_{1}, y_{2}, \ldots, y_{n}\right)^{\top}$ tem distribuição pertencente à classe elíptica se sua função densidade é dada por

$$
f(\mathbf{y})=|\boldsymbol{\Sigma}|^{-\frac{1}{2}} g\left\{(\mathbf{y}-\boldsymbol{\mu})^{\top} \boldsymbol{\Sigma}^{-1}(\mathbf{y}-\boldsymbol{\mu})\right\},
$$

em que $\boldsymbol{\mu} \in \mathbb{R}^{n}, \boldsymbol{\Sigma}$ é uma matriz positiva definida, $|\cdot|$ denota o determinante e $g(\cdot)$ uma função real não negativa que satisfaz a condição

$$
\frac{\pi^{n / 2}}{\Gamma\left(\frac{n}{2}\right)} \int_{0}^{\infty} u^{\frac{n}{2}-1} g(u) d u=1 .
$$

A função $g(\cdot)$ é denominada função geradora de densidades. A condição (1.2) garante que $f(\mathbf{y})$ seja, de fato, uma função densidade (vide Fang, Kotz e $\mathrm{Ng}$; 1990).

O vetor aleatório y com distribuição elíptica tem função característica dada por

$$
\begin{aligned}
\phi_{\mathbf{y}}(\mathbf{t}) & =\mathrm{E}\left[e^{i \mathbf{t}^{\top} \mathbf{y}}\right] \\
& =e^{i \mathbf{t}^{\top} \boldsymbol{\mu}} \psi\left(\mathbf{t}^{\top} \boldsymbol{\Sigma} \mathbf{t}\right),
\end{aligned}
$$

em que $\boldsymbol{\mu} \in \mathbb{R}^{n}, \mathbf{t} \in \mathbb{R}^{n}, \boldsymbol{\Sigma}$ é uma matriz positiva definida e alguma função $\psi, \psi(\cdot) \in \mathbb{R}$. As condições para a função $\psi$ são dadas pelo Teorema 2.2 em Fang, et al. (1990).

Para definir as distribuições elípticas pode-se usar tanto a função característica $\psi(\cdot)$ quanto a função geradora de densidades $g(\cdot)$. Por essa razão, as denotações $\mathbf{y} \sim E C_{n}(\boldsymbol{\mu}, \boldsymbol{\Sigma}, \psi)$ e $\mathbf{y} \sim E C_{n}(\boldsymbol{\mu}, \boldsymbol{\Sigma}, g)$ 
são usadas indiferentemente para indicar que y tem distribuição elíptica. Além disso, diz-se que o vetor aleatório y tem distribuição simétrica multivariada, e denota-se por $\mathbf{y} \sim S_{n}(\phi)$, quando $\mathbf{y} \sim E C_{n}\left(\mathbf{0}, \phi \mathbf{I}_{\mathbf{n}}, g\right)$.

Exemplos clássicos das distribuições elípticas são a distribuição normal multivariada e a distribuição $t$-Student multivariada. Além dessas distribuições, Cysneiros (2004) explorou as distribuições logística I, logística II, logística generalizada, $t$-Student generalizada e exponencial potência. Outros exemplos são as distribuições de Cauchy, potência estendida, Kotz, Kotz generalizada e normal contaminada.

\subsubsection{Propriedades}

A condição (1.2) não garante a existência de momentos para o vetor $\mathbf{y} \sim E C_{n}(\boldsymbol{\mu}, \boldsymbol{\Sigma}, g)$. No entanto, quando existem, a média e a matriz de variância-covariância do vetor y são dadas, respectivamente, por

$$
\mathrm{E}[\mathbf{y}]=\boldsymbol{\mu}
$$

$\mathrm{e}$

$$
\operatorname{Cov}[\mathbf{y}]=\xi \Sigma
$$

em que $\xi=-2 \psi^{\prime}(0)$.

Segue-se que $\boldsymbol{\Sigma}$ coincide com a matriz de variância-covariância apenas quando $\psi^{\prime}(0)=-1 / 2$, a distribuição normal é um exemplo desse fato. Genericamente, $\boldsymbol{\Sigma}$ é proporcional a matriz de variânciacovariância. Porém, a matriz de correlação $\Gamma$ não depende da função característica. As correlações $\rho_{i j}$ são dadas por

$$
\begin{aligned}
\rho_{i j} & =\frac{\operatorname{Cov}\left[y_{i}, y_{j}\right]}{\left\{\operatorname{Var}\left[y_{i}\right] \operatorname{Var}\left[y_{j}\right]\right\}^{\frac{1}{2}}} \\
& =\frac{\xi \sigma_{i j}}{\left\{\xi \sigma_{i i} \xi \sigma_{j j}\right\}^{\frac{1}{2}}} \\
& =\frac{\sigma_{i j}}{\left\{\sigma_{i i} \sigma_{j j}\right\}^{\frac{1}{2}}}
\end{aligned}
$$


em que $\sigma_{i j}$ é um elemento da matriz $\Sigma$. Assim, para cada vetor $\boldsymbol{\mu} \in \mathbb{R}^{n}$ e $\boldsymbol{\Sigma}$ positiva definida fixados, todas as distribuições na classe $\left\{E C_{n}(\boldsymbol{\mu}, \boldsymbol{\Sigma} ; g)\right\}$, com segundo momento finito, além de terem a mesma média também têm a mesma matriz de correlação (vide, por exemplo, Arellano-Valle, 1994). Além disso, de acordo com (1.6), $\Sigma$ especifica as correlações.

Considerando que o segundo momento existe, o coeficiente de correlação é a medida de dependência natural das distribuições elípticas (vide, por exemplo, Mendes, 2004). No entanto, o coeficiente de correlação pode não ser uma boa medida de dependência para distribuições não elípticas, os coeficientes $\tau$ de Kendall e $\rho$ de Spearman são uma alternativa.

Uma boa medida para avaliar as caudas de distribuições simétricas (elípticas) é o coeficiente de curtose. Por exemplo, a distribuição $t$-Student univariada com $\nu$ graus de liberdade tem coeficiente de curtose dado por $\gamma_{2}=3+\frac{6}{\nu-4}$, para $\nu>4$, indicando caudas mais pesadas do que a distribuição normal. O coeficiente de curtose multivariado é definido por

$$
\gamma_{2}=\mathrm{E}\left[\left(\left(\mathbf{y}-\boldsymbol{\mu}_{\mathbf{y}}\right)^{\top} \boldsymbol{\Sigma}_{\mathbf{y}}^{-1}(\mathbf{y}-\boldsymbol{\mu})\right)^{2}\right]
$$

em que $\boldsymbol{\mu}_{\mathbf{y}}=\mathrm{E}[\mathbf{y}]$ e $\boldsymbol{\Sigma}_{\mathbf{y}}=\operatorname{Cov}[\mathbf{y}]$.

Muitas das propriedades da distribuição normal multivariada podem ser estendidas às distribuições elípticas. Uma propriedade muito útil é a seguinte: se $\mathbf{y} \sim E C_{n}(\boldsymbol{\mu}, \boldsymbol{\Sigma}, g)$, então, para uma matriz $(m \times n)$ $\mathbf{A}$ e um vetor $\mathbf{b} \in R^{m}$ tem-se que

$$
\mathbf{x}=\mathbf{A y}+\mathbf{b} \sim E C_{m}\left(\mathbf{A} \boldsymbol{\mu}+\mathbf{b}, \mathbf{A}^{\top} \mathbf{\Sigma} \mathbf{A}, g\right),
$$

ou seja, combinações lineares de distribuições elípticas também pertencem à classe elíptica de distribuições, com a mesma função geradora de densidades. A demonstração desse resultado é imediata, usando-se a função característica de y. Conclui-se ainda, a partir da propriedade (1.8), que a distribuição marginal de qualquer componente de $\mathbf{y}$ também é elíptica. Além disso, todas as distribuições condicionais de $\mathbf{y}$ pertencem à classe elíptica. 
Berkanee e Bentler (1986), Muirhead (1980 e 1982), Rao (1990), Cambanis, Huang e Simons (1981) e Anderson e Fang (1987) discutem essas distribuições.

\subsubsection{Alguns exemplos de distribuições elípticas}

Alguns exemplos de distribuições elípticas são discutidos nesta seção. Os exemplos são apresentados de modo conciso, as diferenças destas distribuições são destacadas graficamente. Uma abordagem mais detalhada pode ser vista em Landsman e Valdez (2002). As distribuições abordadas foram a normal, t-Student, Laplace e Pearson tipo VII. A seção é finalizada comparando-se os resultados obtidos.

\section{Normal}

A distribuição normal é o exemplo mais comum de distribuição pertencente à classe elíptica. O uso freqüente desta distribuição deve-se a todo desenvolvimento teórico e aplicado alcançado.

A função geradora de densidades do vetor aleatório y com distribuição normal é

$$
g(u)=(2 \pi)^{-\frac{n}{2}} e^{-\frac{1}{2} u} .
$$

Portanto, a partir da fórmula (1.1) a densidade multivariada da distribuição normal é dada por

$$
f(\mathbf{y})=\frac{1}{(2 \pi)^{n / 2}}|\boldsymbol{\Sigma}|^{-1 / 2} \exp \left[-\frac{1}{2}(\mathbf{y}-\boldsymbol{\mu})^{\top} \boldsymbol{\Sigma}^{-1}(\mathbf{y}-\boldsymbol{\mu})\right] .
$$

Como é bem conhecido, $\mathrm{E}[\mathbf{y}]=\boldsymbol{\mu}$ e $\operatorname{Cov}[\mathbf{y}]=\boldsymbol{\Sigma}$. Vale ressaltar que $\xi=1$ e, devido a isso, $\boldsymbol{\Sigma}$ coincide com a matriz de variância-covariância.

A Figura 1.1 mostra os gráficos das funções densidade marginal e bivariada da distribuição normal padrão. 

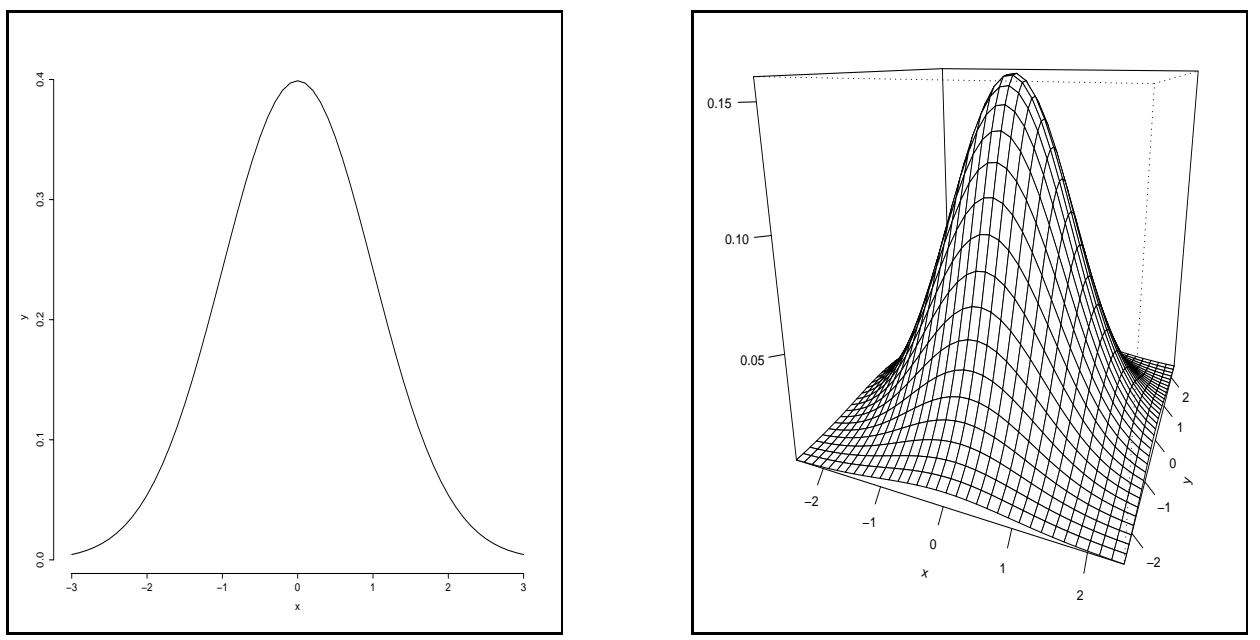

Figura 1.1: Gráficos das funções de densidade marginal e bivariada da distribuição normal padrão.

\section{$t$-Student}

O vetor aleatório y tem distribuição $t$-Student com $\nu$ graus de liberdade, se sua função geradora de densidades é dada por

$$
g(u)=\frac{\Gamma((n+\nu) / 2)}{\Gamma(\nu / 2)(\nu \pi)^{\frac{n}{2}}}\left(1+\frac{u}{\nu}\right)^{-\frac{n+\nu}{2}},
$$

em que $\Gamma(\cdot)$ denota a função gama. Conclui-se, pela fórmula (1.1), que a densidade multivariada da distribuição $t$-Student com $\nu$ graus de liberdades é dada por

$$
f(\mathbf{y})=k|\boldsymbol{\Sigma}|^{-1 / 2}\left[1+\frac{1}{\nu}(\mathbf{y}-\boldsymbol{\mu})^{\top} \boldsymbol{\Sigma}^{-1}(\mathbf{y}-\boldsymbol{\mu})\right]^{-(n+\nu) / 2},
$$

em que $k=\frac{\Gamma((n+\nu) / 2)}{\Gamma(\nu / 2)(\nu \pi)^{n / 2}}$. Tem-se que $\xi=\nu /(\nu-2)$ e, conseqüentemente, $\operatorname{Cov}[\mathbf{y}]=\frac{\nu}{\nu-2} \Sigma, \nu>0$. Vale ressaltar que a distribuição $t$-Student tende à distribuição normal padrão quando $\nu \rightarrow \infty$ e quando $\nu=1$ tem-se a distribuição de Cauchy.

A Figura 1.2 mostra gráficos de densidades univariadas da distribuição $t$ com $\nu=1, \nu=3, \nu=6$ e $\nu=30$ e a densidade bivariada da distribuição $t$-Student com os parâmetros, $\nu=3, \boldsymbol{\mu}=\mathbf{0}$ e $\boldsymbol{\Sigma}=\mathbf{I}$. 

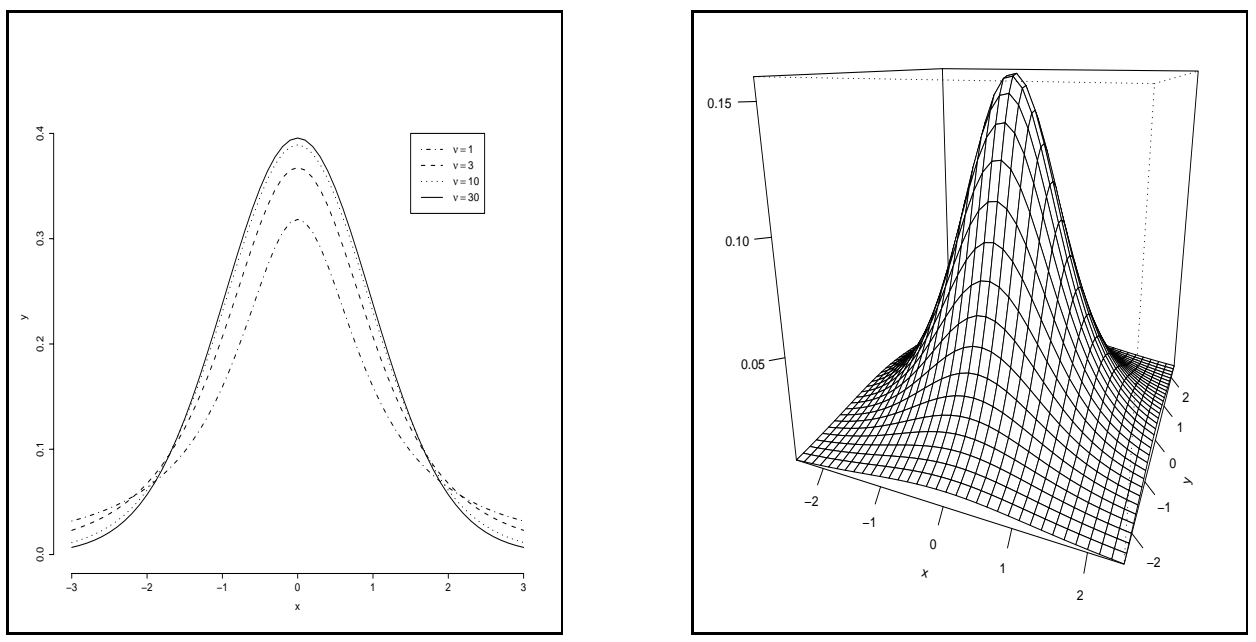

Figura 1.2: Gráficos de funções de densidade marginal e bivariada da distribuição t-Student.

\section{Laplace}

A função geradora de densidades $g(\cdot)$ da distribuição de Laplace ou exponencial dupla é

$$
g_{n}(u)=\frac{\Gamma(n / 2)}{2 \Gamma(n) \pi^{n / 2}} e^{-\sqrt{u}} .
$$

Portanto, a partir de (1.1), o vetor aleatório y com distribuição de Laplace tem distribuição multivariada dada por

$$
f(\mathbf{y})=k|\boldsymbol{\Sigma}|^{-1 / 2} \exp \left[-\left((\mathbf{y}-\boldsymbol{\mu})^{\top} \boldsymbol{\Sigma}^{-1}(\mathbf{y}-\boldsymbol{\mu})\right)^{1 / 2}\right],
$$

em que $k=\frac{\Gamma(n / 2)}{2 \Gamma(n) \pi^{n / 2}}$. Nesse caso, $\xi=2$ e, portanto, $\operatorname{Cov}[\mathbf{y}]=2 \boldsymbol{\Sigma}$.

A Figura 1.3 mostra a função de densidade marginal e bivariada da distribuição Laplace, $\operatorname{com} \boldsymbol{\mu}=\mathbf{0}$ e $\boldsymbol{\Sigma}=\mathbf{I}$.

\section{Pearson tipo VII}

O último exemplo de membros da família elíptica de distribuições é a distribuição Pearson tipo VII, cuja função geradora de 

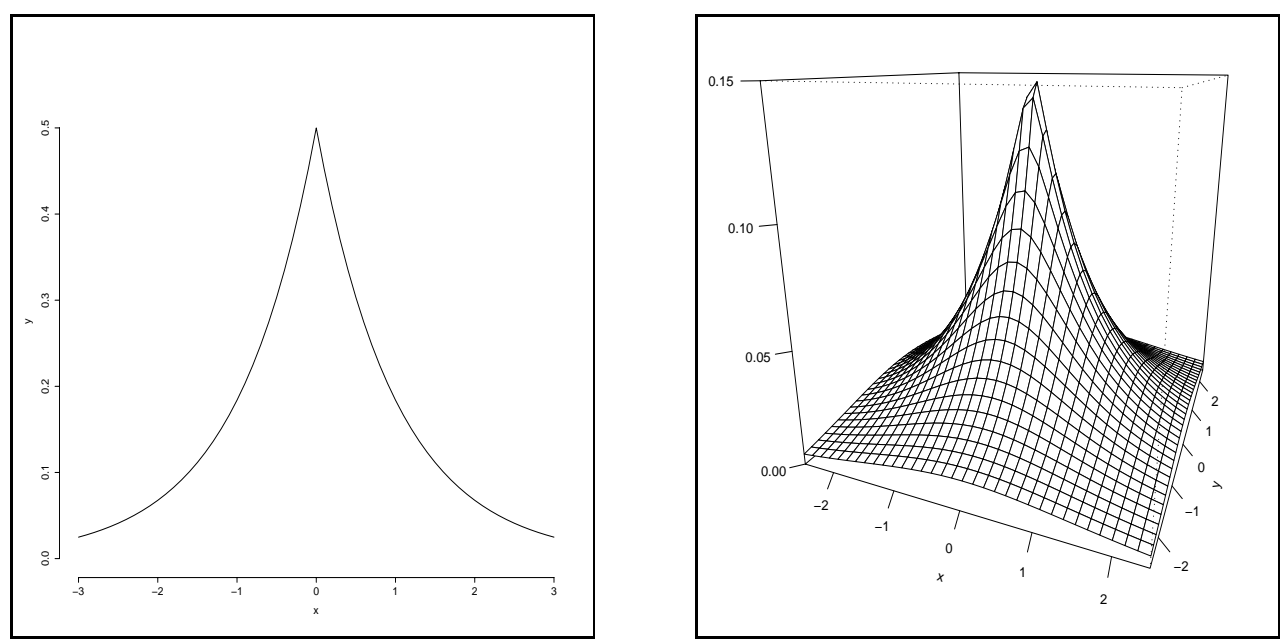

Figura 1.3: Gráficos das funções de densidade marginal e bivariada da distribuição de Laplace.

densidades é dada por

$$
g(u)=\frac{\Gamma(N)}{\Gamma(N-n / 2)(m \pi)^{n / 2}}\left(1+\frac{u}{m}\right)^{-N} .
$$

De modo análogo aos exemplos anteriores, conclui-se que a densidade multivariada dessa distribuição é

$$
f(\mathbf{y})=k|\boldsymbol{\Sigma}|^{-1 / 2}\left(1+\frac{(\mathbf{y}-\boldsymbol{\mu})^{\top} \boldsymbol{\Sigma}^{-1}(\mathbf{y}-\boldsymbol{\mu})}{m}\right)^{-N}
$$

em que $k=\frac{\Gamma(N)}{\Gamma(N-n / 2)(m \pi)^{n / 2}}$. O vetor aleatório $\mathbf{y}$ com distribuição Pearson tipo VII tem $\operatorname{Cov}[\mathbf{y}]=\frac{m}{2 N-n-2} \boldsymbol{\Sigma}$. Ressalta-se que a variância desta distribuição é extremamente flexível, dependendo da parametrização pode-se obter uma variância maior ou menor do que a variância da distribuição $t$-Student, por exemplo. Essa flexibilidade pode ser observada pela Figura 1.4. Tem-se ainda que quando tem se $m$ inteiro e $N=2^{-1}(n+m)$ a distribuição Pearson VII coincide com a distribuição $t$-Student. 

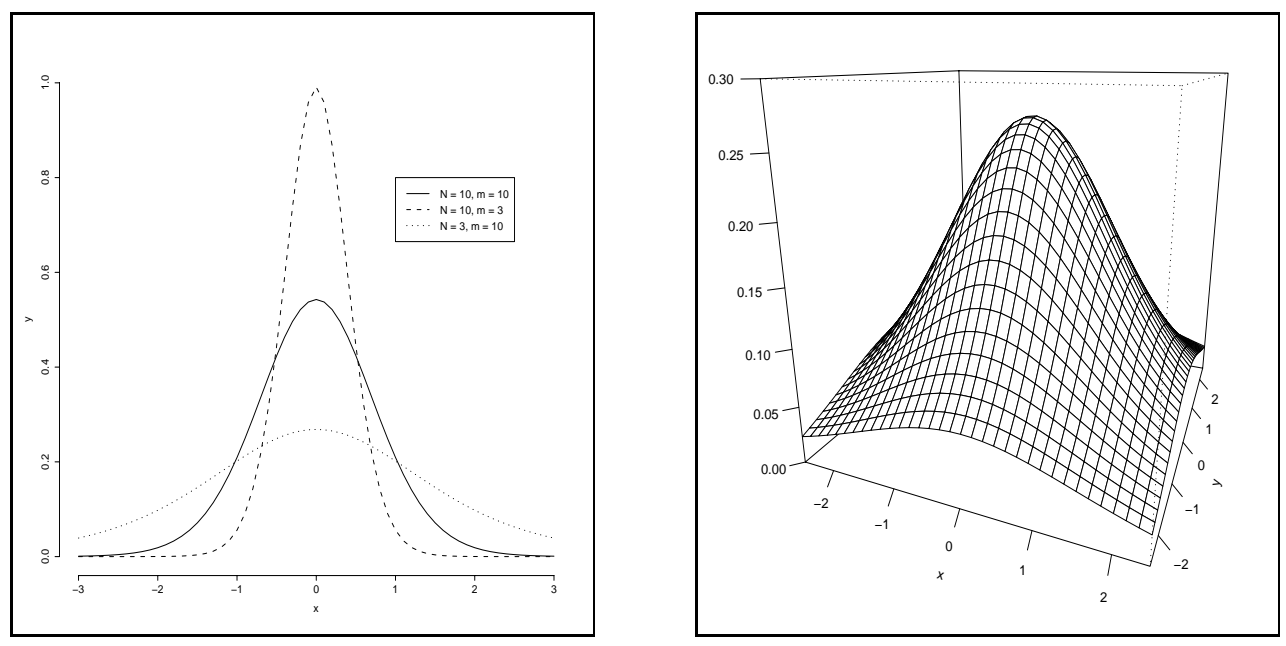

Figura 1.4: Gráficos de funções de densidade marginal e bivariada da distribuição Pearson VII.

\section{Diferenças e similaridades}

Foram apresentados quatro exemplos de distribuições elípticas, a despeito de existirem muitas outras.

Comparando-se as quatro distribuições apresentadas, as diferenças entre elas são facilmente identificadas. Em especial, verifica-se o quanto as caudas são diferentes, que motiva o estudo dessas distribuições ao invés de se preocupar apenas com a distribuição normal.

As Figuras 1.5 e 1.6 mostram, respectivamente, os gráficos e as curvas de nível das distribuições Normal, $t$-Student com $\nu=3$, Laplace e Pearson tipo VII com $N=1$ e $m=10$ padronizadas, isto é, com médias iguais a zero e variâncias iguais a um. As quantidades $\nu, N$ e $m$ foram escolhidas de modo arbitrário. Fica evidente as diferenças entre essas distribuições. Além de mostrar o quanto as distribuições elípticas são flexíveis na modelagem das caudas. 


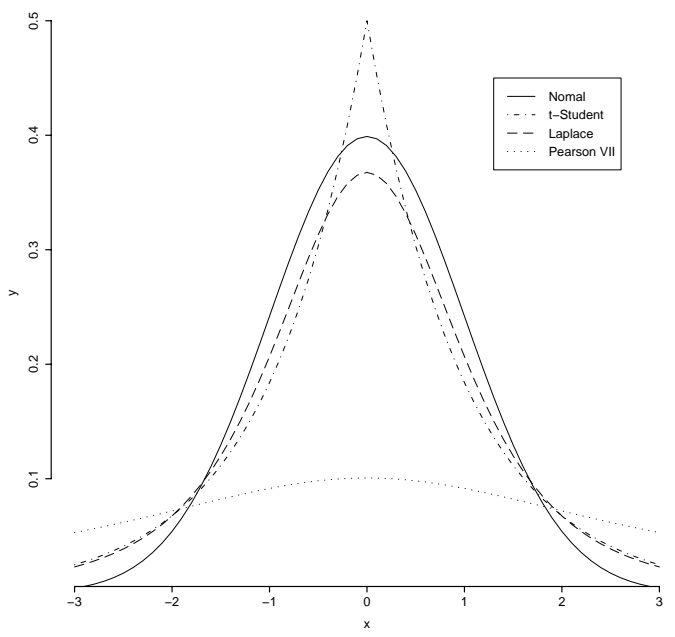

Figura 1.5: Gráficos das distribuições normal padrão, $t$-Student com $\nu=3$, Laplace e Pearson tipo VII com $N=1$ e $m=10$.
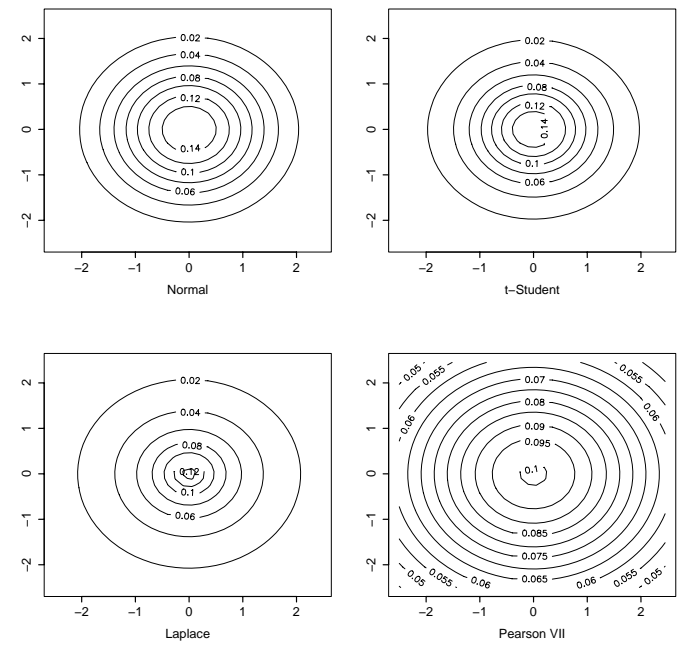

Figura 1.6: Curvas de nível das distribuições normal padrão, $t$-Student com $\nu=3$, Laplace e Pearson tipo VII com $N=1$ e $m=10$. 
Capítulo 2

\section{Modelos simétricos de regressão linear}

Introdução

Análise de regressão é uma metodologia estatística que estuda o relacionamento entre duas ou mais variáveis de modo que uma variável possa ser predita a partir da outra, ou das outras. Um número surpreendentemente grande de problemas pode ser estudado usando análise de regressão normal linear. Em geral, assume-se normalidade para os erros e a técnica de mínimos quadrados para a estimação dos parâmetros. Esta técnica, embora simples, é muito sensível a observações extremas ou aberrantes (outliers), as quais podem causar variações desproporcionais nas estimativas dos parâmetros, na dispersão ou mesmo nas previsões. A suposição de erros com caudas mais pesadas do que a normal conjuntamente com a técnica de máxima verossimilhança pode reduzir a influência de tais observações detectadas sob erros normais, embora possam continuar influenciando, provavel- 
mente com menor intensidade. Sob esses modelos, pode-se dizer que as estimativas de máxima verossimilhança são mais robustas contra observações extremas do que sob erros normais.

Este capítulo descreve brevemente os modelos simétricos de regressão linear. Esses modelos são definidos na Seção 2.1. A Seção 2.2 exibe a função escore e a matriz de informação de Fisher. A estimação dos parâmetros é discutida na Seção 2.3 e o modelo CAPM é usado como exemplo na última seção. O conteúdo do presente capítulo segue, de perto, o Capítulo 2 de Cysneiros (2004).

\section{$2.1 \quad$ Definição do modelo}

Considere as variáveis aleatórias $\epsilon_{1}, \epsilon_{2}, \ldots, \epsilon_{n}$ i.i.d. com densidade pertencente à classe simétrica de distribuições, ou seja, as variáveis têm densidade definida por

$$
f(\epsilon)=\frac{1}{\sqrt{\phi}} g\left(\epsilon^{2} / \phi\right),
$$

em que $\epsilon \in \mathbb{R}$ e $g(\cdot)$ está definida na Seção (1.3). Para a variável resposta $y_{i}$, suponha a relação funcional

$$
y_{i}=\mathbf{x}_{i}^{\top} \boldsymbol{\beta}+\epsilon_{i},
$$

em que $\mathbf{x}_{i}=\left(x_{i 1}, x_{i 2}, \ldots, x_{i p}\right)^{\top}$ é um vetor com os valores de $p$ variáveis explicativas, $p<n, \boldsymbol{\beta}=\left(\beta_{1}, \beta_{2}, \ldots, \beta_{p}\right)^{\top}$ é um vetor $(p \times 1)$ de parâmetros desconhecidos e $\epsilon_{i}$ representa o erro aleatório. Deste modo, a densidade de $y_{i}$ é dada por

$$
f\left(y_{i}\right)=\frac{1}{\sqrt{\phi}} g\left(u_{i}\right)
$$

em que

$$
u_{i}=\frac{\left(y_{i}-\mathbf{x}_{i}^{\top} \boldsymbol{\beta}\right)^{2}}{\phi}
$$


O modelo de regressão definido por (2.2) e (2.3) é chamado modelo simétrico de regressão linear.

Segue-se de $(2.3)$ que $y_{i} \sim S\left(\mathbf{x}_{i}^{\top} \boldsymbol{\beta}, \phi\right)$. Portanto, quando existem, a média e a variância de $y_{i}$ são dadas por

$$
\mathrm{E}\left[y_{i}\right]=\mathbf{x}_{i}^{\top} \boldsymbol{\beta}
$$

e

$$
\operatorname{Var}\left[y_{i}\right]=\xi \phi,
$$

em que $\xi$ é dada na Seção (1.3.1).

2.2 Função escore e informação de Fisher

\subsubsection{Função escore}

Segue-se de (2.3) que o logaritmo da função de verossimilhança de $\boldsymbol{\theta}=\left(\boldsymbol{\beta}^{\top}, \phi\right)^{\top}$ é dado por

$$
\mathbf{L}(\boldsymbol{\theta})=-\frac{n}{2} \log (\phi)+\sum_{i=1}^{n} \log \left\{g\left(u_{i}\right)\right\},
$$

em que $u_{i}=\left(y_{i}-\mathbf{x}_{i}^{\top} \boldsymbol{\beta}\right)^{2} / \phi$. Supõe-se que a função $\mathrm{L}(\boldsymbol{\theta})$ é regular. Algumas distribuições pertencentes à classe elíptica não satisfazem essas condições de regularidade, por exemplo, a exponencial dupla.

Para se obter a função escore e a matriz de informação de Fisher é necessário derivar $\mathrm{L}(\boldsymbol{\theta})$ em relação aos parâmetros desconhecidos e calcular alguns momentos dessas derivadas. Assim, nem sempre é possível calcular essas matrizes. Casos que não satisfazem às condições de regularidades e não têm derivadas ou momentos finitos não foram considerados.

Inicialmente, as quantidades a seguir são definidas supondo $g(\cdot)$ contínua e diferenciável

$$
W_{g}\left(u_{i}\right)=\frac{\partial}{\partial u_{i}} \log \left\{g\left(u_{i}\right)\right\}
$$


e

$$
W_{g}^{\prime}\left(u_{i}\right)=\frac{\partial}{\partial u_{i}} W_{g}\left(u_{i}\right)
$$

A Tabela 2.1 reproduzida de Cysneiros (2004), mostra expressões de $W_{g}\left(u_{i}\right)$ e $W_{g}^{\prime}\left(u_{i}\right)$ para algumas distribuições simétricas.

Após algumas manipulações algébricas, mostra-se que as funções escore para $\boldsymbol{\beta}$ e $\phi$ são dadas, respectivamente, por

$$
\begin{aligned}
\mathbf{U}_{\beta}(\boldsymbol{\theta}) & =\frac{1}{\phi} \mathbf{X}^{\top} \mathbf{D}(\mathbf{v})(\mathbf{y}-\mathbf{X} \boldsymbol{\beta}) \\
\mathrm{e} & \\
\mathrm{U}_{\phi}(\boldsymbol{\theta}) & =\frac{1}{2 \phi}\left(\frac{Q_{v}(\boldsymbol{\beta})}{\phi}-n\right),
\end{aligned}
$$

em que $Q_{v}(\boldsymbol{\beta})=(\mathbf{y}-\mathbf{X} \boldsymbol{\beta})^{\top} \mathbf{D}(\mathbf{v})(\mathbf{y}-\mathbf{X} \boldsymbol{\beta}), \mathbf{D}(\mathbf{v})=\operatorname{diag}\left\{v_{1}, v_{2}, \ldots, v_{n}\right\}$ e $v_{i}=-2 W_{g}\left(u_{i}\right)$.

Tabela 2.1: Expressões para $W_{g}(u)$ e $W_{g}^{\prime}(u)$ para algumas distribuições simétricas.

\begin{tabular}{rcc}
\hline Distribuição & $W_{g}(u)$ & $W_{g}^{\prime}(u)$ \\
\hline Normal & $-\frac{1}{2}$ & 0 \\
t-Student & $-\frac{\nu+1}{2(\nu+u)}$ & $\frac{\nu+1}{2(\nu+u)^{2}}$ \\
t-Student generalizada & $-\frac{r+1}{2(s+u)}$ & $\frac{r+1}{2(s+u)^{2}}$ \\
Logística-I & $-\tanh \left(\frac{u}{2}\right)$ & $-\operatorname{sech}\left(\frac{u}{2}\right) / 2$ \\
Logística-II & $\frac{\exp (-\sqrt{u})-1}{(-2 \sqrt{u}[1+\exp (-\sqrt{u})]}$ & $\frac{2 \exp (-\sqrt{u}) \sqrt{u}+\exp (-2 \sqrt{u})-1}{-4 u^{\frac{3}{2}}[1+\exp (-\sqrt{u})]^{2}}$ \\
Logística generalizada & $\frac{-\alpha m[\exp (-\alpha \sqrt{u})-1]}{(-2 \sqrt{u})[1+\exp (-\alpha \sqrt{u})]}$ & $-\frac{\alpha m}{4} \frac{2 \alpha \exp (-\alpha \sqrt{u}) \sqrt{u}+\exp (-2 \alpha \sqrt{u})-1}{u^{\frac{3}{2}}[1+\exp (-\alpha \sqrt{u})]^{2}}$ \\
Exponencial potência & $-\frac{1}{2(1+k) u^{k / k+1}}$ & $\frac{k}{(1+k)^{2} 2 u^{(2 k+1) /(k+1)}}$ \\
\hline
\end{tabular}




\subsubsection{Informação de Fisher}

Seja $-\left.\ddot{\mathbf{L}}_{\theta \theta}\right|_{\hat{\theta}}$ a matriz de informação de Fisher observada para o vetor de parâmetros $\boldsymbol{\theta}$. Os resultados a seguir são obtidas após de manipulações algébricas.

$$
\ddot{\mathbf{L}}_{\theta \theta}=\left[\begin{array}{cc}
\ddot{\mathbf{L}}_{\beta \beta} & \ddot{\mathbf{L}}_{\beta \phi} \\
\ddot{\mathbf{L}}_{\phi \beta} & \ddot{\mathbf{L}}_{\phi \phi}
\end{array}\right],
$$

em que

$$
\begin{aligned}
\ddot{\mathbf{L}}_{\beta \beta} & =-\frac{1}{\phi} \mathbf{X}^{\top} \mathbf{D}(\mathbf{a}) \mathbf{X}, \\
\ddot{\mathbf{L}}_{\beta \phi} & =\frac{2}{\phi^{2}} \mathbf{X}^{\top} \mathbf{b} \quad \mathrm{e} \\
\ddot{\mathbf{L}}_{\phi \phi} & =\frac{1}{\phi^{2}}\left\{\frac{n}{2}+\mathbf{u}^{\top} \mathbf{D}(\mathbf{c}) \mathbf{u}-\frac{1}{\phi} \mathbf{e}^{\top} \mathbf{D}(\mathbf{v}) \mathbf{e}\right\},
\end{aligned}
$$

sendo $\mathbf{D}(\mathbf{a})=\operatorname{diag}\left\{a_{1}, a_{2}, \ldots, a_{n}\right\}, \mathbf{D}(\mathbf{c})=\operatorname{diag}\left\{c_{1}, c_{2}, \ldots, c_{n}\right\}$, $\mathbf{b}^{\top}=\left(b_{1}, b_{2}, \ldots, b_{n}\right), \mathbf{u}=\left(u_{1}, u_{2}, \ldots, u_{n}\right)^{\top}, a_{i}=\left\{v_{i}-4 W_{g}^{\prime}\left(u_{i}\right) u_{i}\right\}$, $c_{i}=W_{g}^{\prime}\left(u_{i}\right), b_{i}=\left\{W_{g}\left(u_{i}\right)+u_{i} W_{g}^{\prime}\left(u_{i}\right)\right\} e_{i}$ e $e_{i}=\left(y_{i}-x_{i}^{\top} \beta\right), i=1,2, \ldots n$.

A inversa da matriz $\ddot{\mathbf{L}}_{\theta \theta}$ pode ser expressa por

$$
\ddot{\mathbf{L}}_{\theta \theta}^{-1}=\left[\begin{array}{cc}
-\phi \mathbf{M}^{-1}+\frac{\mathbf{A} \mathbf{A}^{\top}}{\mathrm{E}} & \frac{\mathbf{A}}{\mathrm{E}} \\
\frac{\mathbf{A}^{\top}}{\mathrm{E}} & \frac{1}{\mathrm{E}}
\end{array}\right],
$$

em que $\mathbf{M}=\mathbf{X}^{\top} \mathbf{D}(\mathbf{a}) \mathbf{X}, \mathbf{A}=\frac{2}{\phi} \mathbf{M}^{-1} \mathbf{X}^{\top} \mathbf{b}$ e $\mathrm{E}=\ddot{\mathrm{L}}_{\phi \phi}+\frac{2}{\phi^{2}} \mathbf{b}^{\top} \mathbf{X} \mathbf{A}$.

A matriz de informação esperada de Fisher para $\boldsymbol{\theta}$ pode ser expressa na forma

$$
\mathbf{K}_{\theta \theta}=\left[\begin{array}{cc}
\mathbf{K}_{\beta \beta} & 0 \\
0 & \mathbf{K}_{\phi \phi}
\end{array}\right],
$$

em que $\mathbf{K}_{\beta \beta}=\frac{4 d_{g}}{\phi} \mathbf{X}^{\top} \mathbf{X}$ e $\mathrm{K}_{\phi \phi}=\frac{n}{4 \phi^{2}}\left(4 f_{g}-1\right), d_{g}=\mathrm{E}\left[W_{g}^{2}\left(U^{2}\right) U^{2}\right]$, $f_{g}=\mathrm{E}\left[W_{g}^{2}\left(U^{2}\right) U^{4}\right]$ e $U \sim S(1)$. Portanto, $\boldsymbol{\beta}$ e $\phi$ são ortogonais. A 
Tabela 2.2 reproduzida de Cysneiros (2004), mostra valores de $d_{g}$ e $f_{g}$ para algumas distribuições simétricas.

Para $\boldsymbol{\beta} \in \Omega_{\beta} \subset \mathbb{R}^{p}$, em que $\Omega_{\beta}$ é um conjunto aberto com pontos interiores, o estimador de máxima verossimilhança de $\boldsymbol{\beta}$ é, sob certas condições de regularidade, consistente e

$$
\sqrt{n}(\widehat{\boldsymbol{\beta}}-\boldsymbol{\beta}) \stackrel{d}{\rightarrow} N_{p}\left(\mathbf{0}, \mathbf{J}_{\beta \beta}^{-1}\right)
$$

em que $\mathbf{J}_{\beta \beta}=\lim _{n \rightarrow \infty} \frac{\mathbf{K}_{\beta \beta}}{n}$. Então, $\widehat{\mathbf{K}}_{\beta \beta}^{-1}=\frac{\hat{\phi}}{4 d g}\left(\mathbf{X}^{\top} \mathbf{X}\right)^{-1}$ é um estimador consistente da matriz de variância-covariância assintótica de $\widehat{\boldsymbol{\beta}}$. De modo análogo, o estimador de máxima verossimilhança de $\phi$ é, sob certas condições de regularidade, consistente e

$$
\sqrt{n}(\hat{\phi}-\phi) \stackrel{d}{\rightarrow} N\left(0, \mathrm{~J}_{\phi \phi}^{-1}\right)
$$

em que $\mathrm{J}_{\phi \phi}=\lim _{n \rightarrow \infty} \frac{\mathrm{K}_{\phi \phi}}{n}$. Então, $\widehat{\mathrm{K}}_{\phi \phi}^{-1}=\frac{4 \hat{\phi}^{2}}{n\left(4 f_{g}-1\right)}$ é um estimador consistente da variância assintótica de $\hat{\phi}$.

Tabela 2.2: Valores de $d_{g}, f_{g}$ e $\xi$ para algumas distribuições simétricas.

\begin{tabular}{rccc}
\hline Distribuição & $d_{g}$ & $f_{g}$ & $\xi$ \\
\hline Normal & $1 / 4$ & $3 / 4$ & 1 \\
$t$-Student & $\frac{\nu+1}{4(\nu+3)}$ & $\frac{3(\nu+1)}{4(\nu+3)}$ & $\frac{\nu}{\nu-2}, \nu>2$ \\
$t$-Student generalizada & $\frac{r(r+1)}{4 s(r+3)}$ & $\frac{3(r+1)}{4(r+3)}$ & $\frac{s}{r-2}, s>0, r>2$ \\
Logística-I & 0,369310044 & 1,003445984 & 0,79569 \\
Logística-II & $1 / 12$ & 0,60749 & $\pi^{2} / 3$ \\
Exponencial potência & $\frac{\Gamma\{(3-k) / 2\}}{4\left(2^{k-1}\right)(1+k)^{2} \Gamma\{(k+1) / 2\}}$ & $\frac{k+3}{4(k+1)}$ & $\frac{\Gamma\{3(k+1) / 2\}}{\Gamma\{(k+1) / 2\}}$ \\
\hline
\end{tabular}




\section{Estimação}

Um processo iterativo para obter as estimativas de máxima verossimilhança de $\boldsymbol{\beta}$ e $\phi$ pode ser desenvolvido a partir das equações $\mathbf{U}_{\beta}(\boldsymbol{\theta})=\mathbf{0}$ e $\mathrm{U}_{\phi}(\boldsymbol{\theta})=0$. O processo conjunto é dado por

$$
\begin{gathered}
\boldsymbol{\beta}^{(m+1)}=\left\{\mathbf{X}^{\top} \mathbf{D}\left(\mathbf{v}^{(m)}\right) \mathbf{X}\right\}^{-1} \mathbf{X}^{\top} \mathbf{D}\left(\mathbf{v}^{(m)}\right) \mathbf{y} \\
\mathrm{e} \\
\phi^{(m+1)}=\frac{Q_{v}\left(\boldsymbol{\beta}^{m+1}\right)}{n}, m=0,1, \ldots,
\end{gathered}
$$

em que $Q_{v}(\boldsymbol{\beta})=(\mathbf{y}-\mathbf{X} \boldsymbol{\beta})^{\top} \mathbf{D}(\mathbf{v})(\mathbf{y}-\mathbf{X} \boldsymbol{\beta}), \mathbf{D}(\mathbf{v})=\operatorname{diag}\left\{v_{1}, v_{2}, \ldots, v_{n}\right\}$ e $v_{i}=-2 W_{g}\left(u_{i}\right)$.

O processo iterativo (2.14) pode ser interpretado como um procedimento de mínimos quadrados reponderado com pesos $v_{1}, v_{2}, \ldots, v_{n}$. Esses pesos (vide Tabela 2.1) são em geral inversamente proporcionais a $u_{i}=\left(y_{i}-\mathbf{x}_{i}^{\top} \boldsymbol{\beta}\right)^{2} / \phi$ de modo que pontos aberrantes (segundo $u_{i}$ ) tenham um peso menor no processo de estimação. O mesmo ocorre na estimação de $\phi$ que é ponderada pelos pesos $v_{1}, v_{2}, \ldots, v_{n}$, segundo o processo iterativo (2.15). Assim, pode-se dizer que o método de máxima verossimilhança produz estimativas robustas (no sentido de $u_{i}$ ) na classe de modelos simétricos lineares com erros com caudas mais pesadas do que a normal. 
$2.4 \quad$ Exemplo

2.4.1 Modelo CAPM das ações da Telemar

\section{Motivação}

Investimentos sem risco caracterizam-se por oferecerem um retorno conhecido e teoricamente não há chance desse retorno não se concretizar. Os títulos do governo são os melhores investimentos sem risco, pois espera-se que o governo possa honrar sua promessa, embora exista o risco de inflação. A taxa de retorno desses investimentos é conhecida como taxa de juros sem riscos (risk-free rate). No entanto, investimentos de capital geralmente oferecem risco e esse risco precisa ser avaliado e previsto adequadamente (vide, por exemplo, Elton e Gruber; 1995).

Segue-se que um projeto de capital sem risco deve render uma taxa de retorno que seja pelo menos igual à taxa de juros sem riscos. Os projetos de investimentos de risco são avaliados a partir de uma taxa de retorno requerida por referência a um investimento semelhante no mercado de ações. Para tanto, é necessário examinar o que o risco significa nesse mercado.

Investidores não gostam de se expor a riscos a menos que eles possam esperar o recebimento de uma compensação pela sua exposição. Uma comparação interessante, então, seria feita entre o retorno do risco sobre a ação e a taxa de juros sem risco. A diferença entre essas duas taxas de retorno se chama retorno excedente ou prêmio do risco de ação. Ele mede o retorno adicional recebido do investimento em participações, em vez de notas do tesouro. Se o retorno excedente for em média, positivo, então os investidores estão recebendo um prêmio pela exposição ao risco no mercado de ações.

Projetos menos arriscados, portanto, devem ter seus fluxos de caixa descontados à taxa de juros sem risco. Se o prêmio de risco no futuro for semelhante ao seu valor médio no passado, então os projetos 
cujo risco é igual a investir no mercado de ações devem ter seus fluxos de caixa descontados à taxa sem risco mais uma taxa estabelecida, por exemplo: oito por cento. Um projeto com risco intermediário merece uma taxa de desconto intermediária.

\section{Modelo CAPM}

Para implementar a avaliação de um projeto de capital baseada na comparação entre os retornos esperados do projeto e os retornos assegurados pelos títulos do governo é necessário estimar a qualidade de risco de um investimento. Até os anos 60 isso teria sido difícil. Mas na primeira parte daquela década houve um avanço importante na teoria das finanças. Os modelos de precificação de ativos (CAPM) desenvolvidos por Sharpe (1964), Lintner (1965) e Mossin (1966) permitiram relacionar o retorno esperado sobre um ativo financeiro ao seu risco, dando uma definição precisa para o significado de risco.

Particularmente, pode-se definir um dos modelos CAPM da seguinte maneira:

$$
R_{t}-r_{f t}=\alpha+\beta\left(R_{M t}-r_{f t}\right)+\epsilon_{t}
$$

em que $R_{t}$ é o retorno de um ativo entre os períodos de tempo $t \mathrm{e}$ $(t-1), R_{M t}$ é o retorno de um índice de mercado entre os períodos $t$ e $(t-1), r_{f t}$ denota a taxa de retorno entre os períodos $t$ e $(t-1)$ de um ativo livre de risco e $\epsilon_{t}$ representa o erro aleatório. Usualmente, assume-se que este erro é um ruído branco com distribuição normal.

A regressão (2.16) pode ser redefinida como

$$
y_{t}=\alpha+\beta x_{t}+\epsilon_{t}
$$

em que $y_{t}=R_{t}-r_{f t}$ e $x_{t}=R_{M t}-r_{f t}$. As quantidades $y_{t}$ e $x_{t}$ são denominadas excessos de retornos.

A principal idéia do CAPM é que os investidores podem esperar uma recompensa pela contribuição de um investimento para o risco de um portfólio. Não pode haver nenhuma recompensa esperada 
para a exposição aos riscos que são diversificadas facilmente. A taxa de retorno requerida deve ser maior para investimentos com elementos maior de risco não diversificável.

\section{Risco - Interpretação de $\beta$}

Um portfólio investido em apenas uma participação é geralmente muito mais volátil do que um portfólio diversificado. Mantendo um número grande de títulos mobiliários, os investimentos podem eliminar o risco específico para a companhia. Entretanto, existem limites para o poder da diversificação, mesmo que o investidor tenha controle sobre cada ação do mercado. Embora a diversificação possa eliminar o risco específico para a companhia, ela não pode eliminar o risco geral do mercado de ações.

Toda ação, portanto, flutua em valor por causa dos dois elementos de risco. O primeiro, é o risco de mercado - a tendência da ação mudar junto com a movimentação geral do mercado de ações. O segundo é o risco específico, o qual encapsula todos os eventos que são específicos das companhias individuais, embora nada tenha a ver com os fatores gerais no nível do mercado. Os investidores não gostam de risco e precisam da perspectiva de retornos altos antes de assumirem o risco. Como o risco de mercado não pode ser evitado por meio da diversificação, os investidores exigem um retorno mais alto para a exposição ao mercado de risco. No CAPM, o risco de mercado ou risco sistemático é medido por $\beta$. Uma ação para qual $\beta$ não é significativamente diferente de 1,0 deve se mover em concordância com o mercado. Quando $\beta$ é significativamente maior do que 1, 0 esta ação é mais agressiva do que o mercado e quando $\beta$ é significativamente menor do que 1, 0 esta ação é conservadora em relação ao mercado.

\section{Aplicação}

Para estimar os parâmetros da regressão (2.17) o método de mínimos quadrados é geralmente usado. Mais ainda, para se fazer 
inferências, admite-se que os excessos de retornos têm distribuição normal. No entanto, como se sabe essa suposição é sensível a retornos atípicos, que acontecem com freqüência no mundo real, especialmente na América Latina (vide, por exemplo, Cademartori, Romo, Campos e Galea; 2003 e Cysneiros, Paula e Galea; 2005). Em outras palavras, o método de mínimos quadrados - ou equivalentemente o método de máxima verossimilhança sob a suposição de normalidade - não é o procedimento recomendado na presença de outliers. A abordagem adotada para contornar esse problema é identificar uma distribuição simétrica com caudas mais pesadas do que a distribuição normal, a fim de reduzir a influência dos pontos aberrantes nas estimativas de máxima verossimilhança. Ou seja, assume-se que o erro $\epsilon_{t}$ da regressão (2.17) tem distribuição $S(0, \phi)$.

Duas estimativas da regressão (2.17) para a Telemar são apresentadas a seguir. Inicialmente, são apresentadas as estimativas baseadas na suposição de normalidade e em seguida mostram-se as estimativas supondo distribuição $t$-Student com 3 graus de liberdade para os erros. Os dados usados são de outubro de 1998, quando a Telemar começou a negociar, a agosto de 2004. O índice usado como proxy do mercado foi o Ibovespa, e o CDI 30 nominal foi usado como taxa livre de risco.

A Figura 2.1 mostra os excessos de retornos mensais da Telemar e do Ibovespa. Os excessos de retornos parecem estacionários e ergóticos, bem como, tendem a se mover na mesma direção ao longo do tempo.

Os parâmetros estimados para o modelo CAPM da Telemar usando a suposição de normalidade são dados pela Tabela 2.3.

Tabela 2.3: Estimativas dos parâmetros do modelo CAPM supondo distribuição normal para os erros.

\begin{tabular}{crrrr}
\hline Coeficiente & Valor & Erro padrão & Z-valor & P-valor \\
\hline$\alpha$ & $-0,0025$ & 0,0559 & $-0,0450$ & 0,9641 \\
$\beta$ & 0,9959 & 0,0488 & 20,4256 & $<0,0001$ \\
$\phi$ & 0,0041 & 0,0007 & & \\
\hline
\end{tabular}



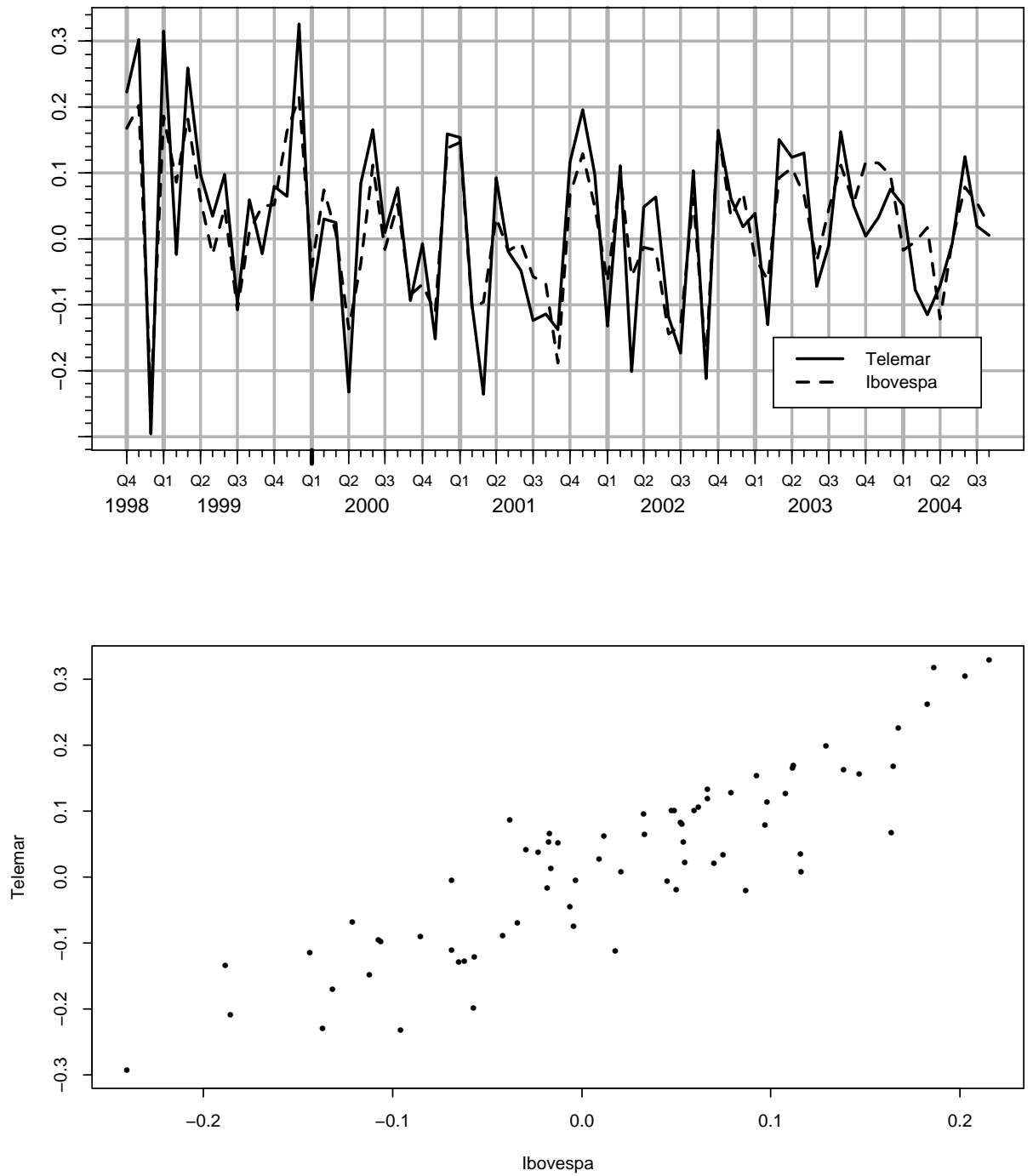

Figura 2.1: Retornos mensais da Telemar e do Ibovespa. 
A estimativa para $\beta$ da Telemar é 0, 9956 com um erro padrão estimado de 0,0488 . Um intervalo de confiança com $95 \%$ de confiança é dado por $(0,9003 ; 1,0914)$. Portanto, a Telemar pode ser julgada como tendo o mesmo comportamento que o Ibovespa. A estimativa para $\alpha$ é -0,0025, com p-valor igual a 0,9641, assim pode-se considerar que o CAPM da Telemar tem $\alpha$ igual a zero. Isto significa que os preços das ações, da Telemar se comportam conforme o mercado. O $\phi$ estimado é 0,0041 , com erro padrão igual a 0,0007. O $\phi$ é interpretado como sendo a magnitude de risco diversificável, nesse caso, portanto, 4,1\%.

A Figura 2.2 mostra o diagrama de dispersão e a reta ajustada supondo que os erros têm distribuição normal.

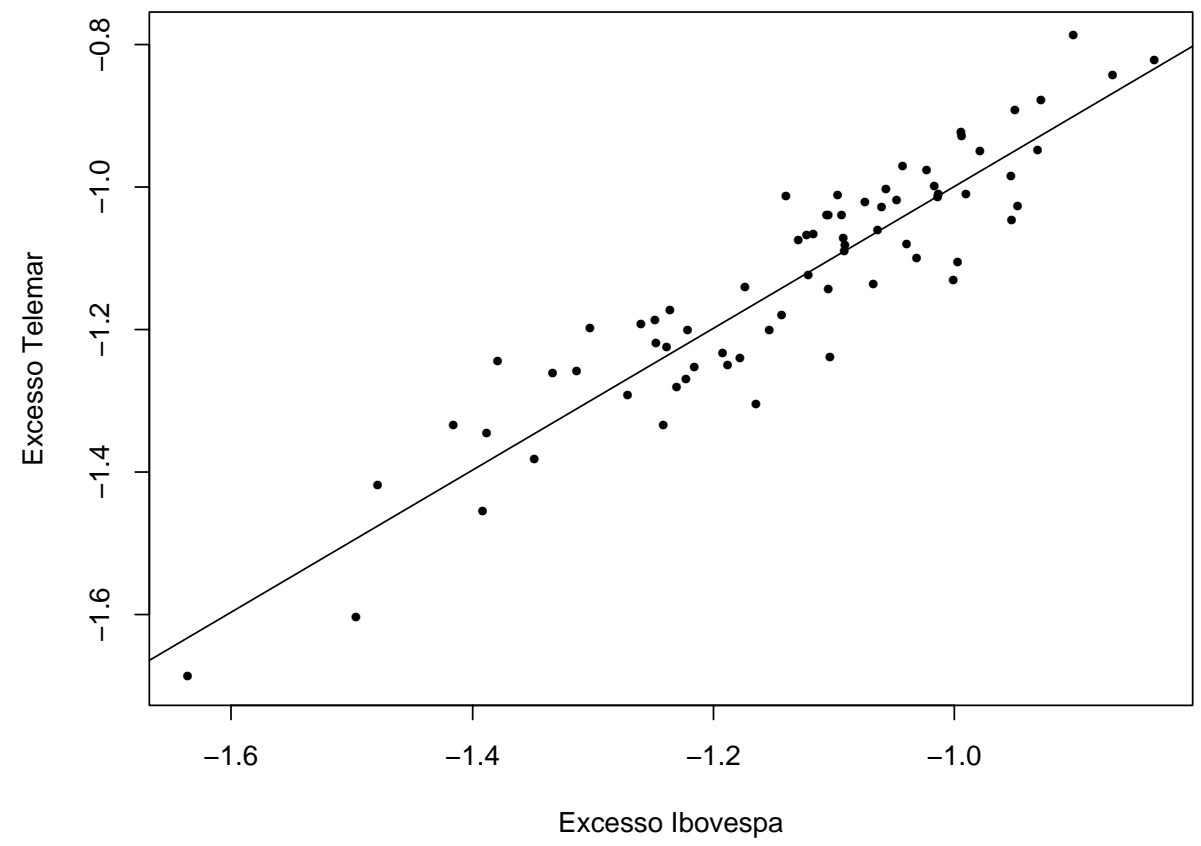

Figura 2.2: Reta ajustada, supondo distribuição normal para os erros, e gráfico de dispersão entre os excessos de retorno do Ibovespa e Telemar. 
Os parâmetros estimados para o modelo CAPM da Telemar usando a distribuição $t$-Student com 3 graus de liberdade são dados pela Tabela 2.4. A Figura 2.3 mostra a reta ajustada para esta suposição.

Tabela 2.4: Estimativas dos parâmetros do modelo CAPM supondo distribuição $t$-Student com 3 graus de liberdade para os erros.

\begin{tabular}{crrrr}
\hline Coeficiente & Valor & Erro padrão & Z-valor & P-valor \\
\hline$\alpha$ & $-0,0160$ & 0,0569 & $-0,2819$ & 0,7780 \\
$\beta$ & 1,0070 & 0,0497 & 20,2649 & $<0,0001$ \\
$\phi$ & 0,0029 & 0,0007 & & \\
\hline
\end{tabular}

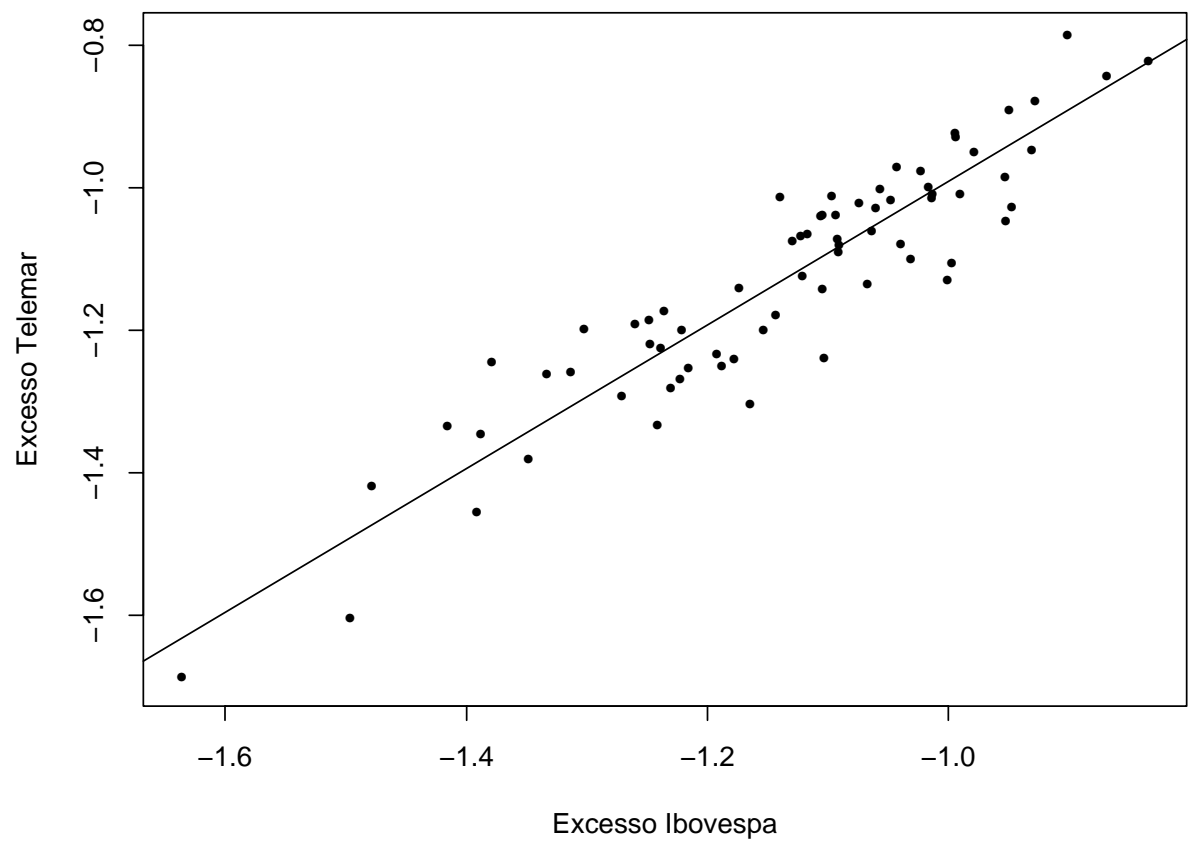

Figura 2.3: Reta ajustada, supondo distribuição $t$-Student com 3 g.l. para os erros, e gráfico de dispersão entre os excessos de retorno do Ibovespa e Telemar. 
Os modelos são comparados através da Figura 2.4. Preliminarmente, os dois modelos parecem adequados, não há indícios de problemas com o ajuste. No entanto, é necessário uma análise de diagnóstico antes de optar por um dos modelos.

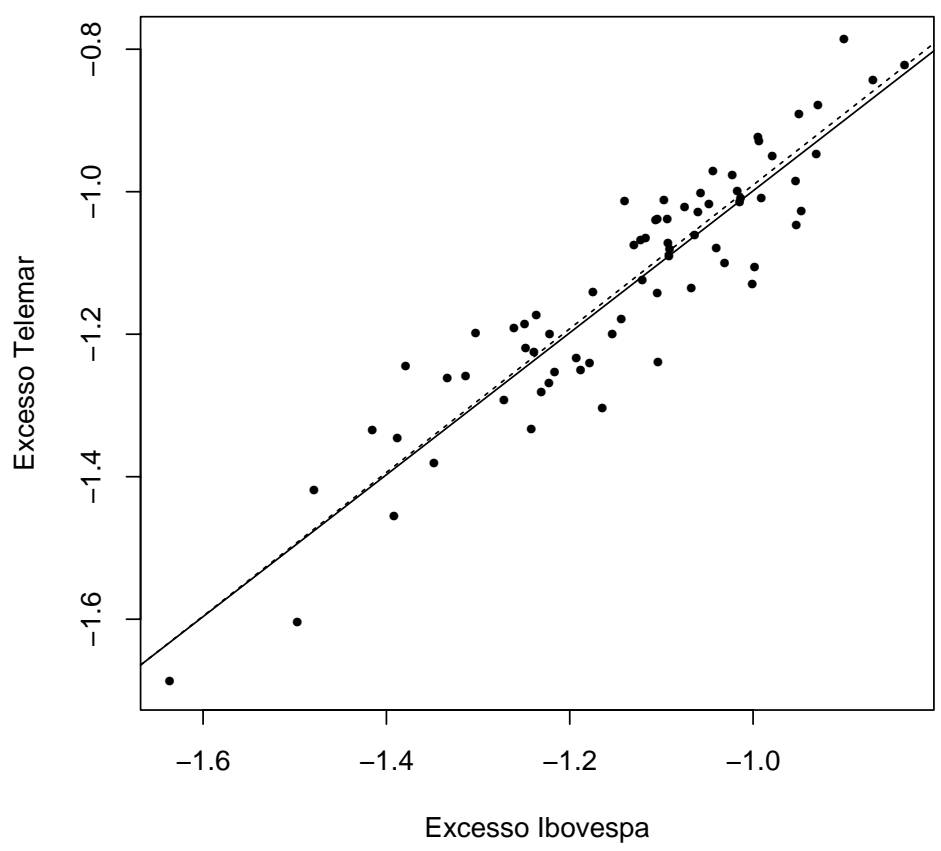

Figura 2.4: Retas ajustadas de acordo com as suposições para os erros e gráfico de dispersão entre os excessos de retorno do Ibovespa e Telemar. 
Capítulo 3

\section{Modelos simétricos de regressão linear com erros $\mathrm{AR}(1)$}

Introdução

Os modelos de regressão geralmente assumem que os erros $\epsilon_{t}$, $t=1,2, \ldots, T$, são variáveis aleatórias não correlacionadas normais independentes . No entanto, pode haver uma substancial correlação nos erros para dados coletados seqüencialmente ao longo do tempo. Isso geralmente acontece em dados econômicos em que a resposta $\mathbf{y}$ e as variáveis explicativas $\mathbf{x}_{t}=\left(x_{t 1}, x_{t 2}, \ldots, x_{t p}\right)^{\top}$ medem o estado de um mercado em um determinado tempo. Nessa situação, os erros são freqüentemente correlacionados positivamente ao longo do tempo e portanto, tanto y quanto $\mathbf{x}$ são séries temporais. Além disso, em finanças, por exemplo, freqüentemente observa-se dados com a presença de observações extremas e, como se sabe, as estimativas dos parâmetros da regressão mostram-se sensíveis a tais observações. Desse modo, além de incorporar, nos modelos de regressão, uma estrutura de correlação 
é necessário supor uma distribuição para os erros com caudas mais pesadas do que a distribuição normal. Erros correlacionados ao longo do tempo são ditos autocorrelacionados ou serialmente correlacionados. Outro exemplo bem conhecido na literatura de modelos com erros autocorrelacionados são os modelos de curvas de crescimento.

Existem muitas fontes de autocorrelação. No entanto, possivelmente a maior causa de autocorrelação no erro envolvendo dados em séries temporais é devido à omissão de uma ou mais variáveis no modelo (Montgomery et al., 2001). Dados de economia, quando uma variável explicativa é omitida, normalmente apresentam correlação positiva nos erros (Neter et al., 1996).

Quando o erro no modelo de regressão tem autocorrelação positiva, o uso de procedimentos de mínimos quadrados tem conseqüências importantes (vide, por exemplo, Thejll e Schmith, 2005):

- Os coeficientes estimados da regressão são ainda não viesados, mas não têm variância mínima;

- A variância pode ser subestimada, conseqüentemente os erros padrão das estimativas dos coeficientes da regressão serão pequenos;

- Intervalos de confiança e testes de hipóteses usando as estatísticas $t$ e $F$ não são aplicáveis.

Como observado anteriormente, uma grande causa para os erros serem autocorrelacionados é a omissão de uma ou mais variáveis explicativas que estejam ordenadas no tempo. Quando o erro autocorrelacionado estiver presente, a primeira medida é procurar variáveis ausentes no modelo. Caso persista a autocorrelação na variável resposta uma componente de tendência deve ser adicionada ao modelo de regressão, como uma tendência linear ou exponencial. O uso de variáveis indicadoras para efeito sazonal pode ser útil para eliminar ou reduzir a autocorrelação no erro quando a variável resposta tem efeito sazonal. Somente quando o uso adicional de variáveis não é útil para 
contornar esse problema deve-se estimar o parâmetro de autocorrelação $\rho$.

Neste trabalho supõem-se que as variáveis do modelo estejam bem especificadas. Ou seja, o problema da autocorrelação é devido ao fenômeno estudado. Portanto, para tratar o problema é necessário estimar $\rho$.

Este capítulo descreve os modelos simétricos de regressão linear com erros AR(1). Esse modelo é definido na Seção 3.1. A Seção 3.2 exibe a função escore e a matriz de informação de Fisher. A Seção 3.3 trata da estimação do modelo e como exemplo, novamente, o modelo CAPM é discutido na última seção.

\subsection{Definição do modelo}

Considere as variáveis aleatórias $\delta_{1}, \delta_{2}, \ldots, \delta_{T}$ i.i.d. com densidade pertencente à classe simétrica de distribuições, ou seja, densidade definida por

$$
f(\delta)=\frac{1}{\sqrt{\phi}} g\left(\delta^{2} / \phi\right),
$$

em que $\delta \in \mathbb{R}$ e $g(\cdot)$ definida na Seção 1.3. Para a variável resposta $y_{i}$, suponha a relação funcional

$$
\begin{aligned}
y_{t} & =\mathbf{x}_{t}^{\top} \boldsymbol{\beta}+\epsilon_{t} \quad \mathrm{e} \\
\epsilon_{t} & =\rho \epsilon_{t-1}+\delta_{t},
\end{aligned}
$$

em que $\mathbf{x}_{t}=\left(x_{t 1}, x_{t 2}, \ldots, x_{t p}\right)^{\top}$ é um vetor com valores de $p$ variáveis explicativas, $p<T, \boldsymbol{\beta}=\left(\beta_{1}, \beta_{2}, \ldots, \beta_{p}\right)^{\top}$ um vetor de parâmetros desconhecidos e $\epsilon_{t}$ o erro aleatório.

O erro $\epsilon_{t}$ consiste em uma fração do erro anterior (quando $\rho>0)$ mais um novo choque $\delta_{t}$. Assim, $\epsilon_{t}$ pode ser escrito como:

$$
\epsilon_{t}=\sum_{s=0}^{\infty} \rho^{s} \delta_{t-s}
$$


permitindo outra interpretação, o erro $\epsilon_{t}$ no período $t$ é uma combinação linear do choque atual precedida dos choques anteriores. Como $|\rho|<1$, quanto maior o período $\tau$ menor é o peso da perturbação $\varepsilon_{t-\tau}$ para determinar $\epsilon_{t}$.

Assumindo-se que a matriz de variância-covariância de y seja dada por

$$
\operatorname{Cov}[\mathbf{y}, \mathbf{y}]_{T \times T}=\frac{\xi \phi}{1-\rho^{2}}\left[\begin{array}{cccccc}
1 & \rho & \rho^{2} & \cdots & \rho^{T-2} & \rho^{T-1} \\
\rho & & \rho & \cdots & \rho^{T-3} & \rho^{T-2} \\
\rho^{2} & \rho & 1 & \cdots & \rho^{T-4} & \rho^{T-3} \\
\vdots & \vdots & \vdots & \ddots & \vdots & \vdots \\
\rho^{T-1} & \rho^{T-2} & \rho^{T-3} & \cdots & \rho & 1
\end{array}\right]
$$

Tem-se que a densidade do vetor de respostas observadas, y, é dada por

$$
f(\mathbf{y})=|\Sigma|^{-1 / 2} g(u),
$$

em que

$$
u=(\mathbf{y}-\mathbf{X} \boldsymbol{\beta})^{\top} \boldsymbol{\Sigma}^{-1}(\mathbf{y}-\mathbf{X} \boldsymbol{\beta}),
$$

sendo $\mathbf{y}$ o vetor resposta, $\mathbf{X}$ uma matriz $(T \times p)$ com os valores das $p$ variáveis explicativas, $p<T$, e $\boldsymbol{\Sigma}$ é proporcional a matriz escala positiva definida definida por (3.4).

O modelo definido por (3.2) - (3.5) é dito simétrico de regressão linear com erros AR(1). Esse modelo de regressão é igual ao modelo definido por (2.2) e (2.3), exceto para a estrutura do erro. Tem-se que esse modelo é uma regressão mais geral, pois os erros são correlacionados. No entanto, quando $\rho=0$ o vetor de respostas $\mathbf{y}$ tem variáveis não autocorrelacionadas e não necessariamente independentes, ou seja, não basta fazer $\rho=0$ para se obter o modelo definido por (2.2) e (2.3).

Em muitas aplicações, espera-se que a autocorrelação decresça firmemente quando a "distância" $\tau$ entre as respostas $y_{t}$ e $y_{t-\tau}$ cresce, de modo que todas as respostas são correlacionadas, mas quanto maior a distância entre elas menos correlacionadas elas são. O modelo de regressão (3.2) - (3.5) reflete essa característica. 
3.2 Função escore e informação de Fisher

\subsubsection{Função escore}

Segue-se da função de densidade (3.5) que o logaritmo da função de verossimilhança de $\boldsymbol{\theta}=\left(\boldsymbol{\beta}^{\top}, \phi, \rho\right)^{\top}$ é dado por

$$
\mathbf{L}(\boldsymbol{\theta})=-\frac{1}{2} \log (|\boldsymbol{\Sigma}|)+\log \{g(u)\}
$$

De modo análogo à Seção 2.2, a função $\mathrm{L}(\boldsymbol{\theta})$ é assumida ser regular e não foram consideras as distribuições elípticas que não têm derivadas em relação aos parâmetros desconhecidos da função $L(\boldsymbol{\theta})$ ou alguns momentos dessas derivadas.

Inicialmente, as quantidades a seguir são definidas supondo $g$ contínua e diferenciável

$$
\begin{aligned}
W_{g}(u) & =\frac{\partial}{\partial u} \log \{g(u)\} \\
\mathrm{e} & \\
W_{g}^{\prime}(u) & =\frac{\partial}{\partial u} W_{g}(u) .
\end{aligned}
$$

A Tabela 3.1 mostra as expressões de $W_{g}(u)$ e $W_{g}^{\prime}(u)$ para algumas distribuições.

A partir das propriedades da Seção 1.3 .1 e da expressão (3.4) a matriz de escala $\Sigma$ para o caso $\mathrm{AR}(1)$ pode ser expressa por

$$
\Sigma=\phi \mathbf{V}
$$


em que

$$
\mathbf{V}=\frac{1}{1-\rho^{2}}\left[\begin{array}{cccccc}
1 & \rho & \rho^{2} & \cdots & \rho^{T-2} & \rho^{T-1} \\
\rho & 1 & \rho & \cdots & \rho^{T-3} & \rho^{T-2} \\
\rho^{2} & \rho & 1 & \cdots & \rho^{T-4} & \rho^{T-3} \\
\vdots & \vdots & \vdots & \ddots & \vdots & \vdots \\
\rho^{T-1} & \rho^{T-2} & \rho^{T-3} & \cdots & \rho & 1
\end{array}\right] .
$$

Mostra-se que

$$
\mathbf{V}^{-1}=\mathbf{L}^{\top} \mathbf{L}
$$

em que

$$
\mathbf{L}=\left[\begin{array}{cccccc}
\sqrt{1-\rho^{2}} & 0 & 0 & \cdots & 0 & 0 \\
-\rho & 1 & 0 & \cdots & 0 & 0 \\
0 & -\rho & 1 & \cdots & 0 & 0 \\
\vdots & \vdots & \vdots & \ddots & \vdots & \vdots \\
0 & 0 & 0 & \cdots & -\rho & 1
\end{array}\right]
$$

Conseqüentemente, de (3.8)

$$
\boldsymbol{\Sigma}^{-1}=\frac{1}{\phi} \mathbf{L}^{\top} \mathbf{L}
$$

Sabendo-se que $\left|\mathbf{A}^{-1}\right|=|\mathbf{A}|^{-1}$ (vide, por exemplo, Harville; 1997, Magnus e Neudecker; 1997) e substituindo (3.11) em (3.7), mostrase que o logaritmo da função de verossimilhança fica dado por

$$
\mathbf{L}(\boldsymbol{\theta})=-\frac{T}{2} \log (\phi)+\frac{1}{2} \log \left(1-\rho^{2}\right)+\log \{g(u)\}
$$

e (3.6) pode ser reescrito como

$$
\begin{aligned}
u & =(\mathbf{y}-\mathbf{X} \boldsymbol{\beta})^{\top} \frac{1}{\phi} \mathbf{L}^{\top} \mathbf{L}(\mathbf{y}-\mathbf{X} \boldsymbol{\beta}) \\
& =\frac{1}{\phi}[\mathbf{L}(\mathbf{y}-\mathbf{X} \boldsymbol{\beta})]^{\top}[\mathbf{L}(\mathbf{y}-\mathbf{X} \boldsymbol{\beta})]
\end{aligned}
$$


De acordo com os resultados do Apêndice A, as funções escore para $\boldsymbol{\beta}, \phi$ e $\rho$ são dadas, respectivamente, por

$$
\begin{aligned}
\mathbf{U}_{\beta}(\boldsymbol{\theta}) & =\frac{v}{\phi} \mathbf{X}^{\top} \mathbf{L}^{\top} \mathbf{L}(\mathbf{y}-\mathbf{X} \boldsymbol{\beta}) \\
\mathbf{U}_{\phi}(\boldsymbol{\theta}) & =\frac{1}{2 \phi}\left\{\phi^{-1} Q_{v}(\boldsymbol{\beta})-T\right\} \\
& \mathrm{e} \\
\mathbf{U}_{\rho}(\boldsymbol{\theta}) & =-\frac{\rho}{1-\rho^{2}}+\frac{W_{g}(u)}{\phi} \mathbf{e}^{\top} \frac{\partial \mathbf{L}^{\top} \mathbf{L}}{\partial \rho} \mathbf{e}
\end{aligned}
$$

em que $v=-2 W_{g}(u), Q_{v}(\boldsymbol{\beta})=v(\mathbf{y}-\mathbf{X} \boldsymbol{\beta})^{\top} \mathbf{L}^{\top} \mathbf{L}(\mathbf{y}-\mathbf{X} \boldsymbol{\beta})$, $u$ é dado em (3.13), $\mathbf{e}=(\mathbf{y}-\mathbf{X} \boldsymbol{\beta})$ e

$$
\frac{\partial \mathbf{L}^{\top} \mathbf{L}}{\partial \rho}=\left[\begin{array}{ccccccc}
0 & -1 & 0 & 0 & \cdots & 0 & 0 \\
-1 & 2 \rho & -1 & 0 & \cdots & 0 & 0 \\
0 & -1 & 2 \rho & -1 & \cdots & 0 & 0 \\
\vdots & \vdots & \vdots & \vdots & \ddots & \vdots & \vdots \\
0 & 0 & 0 & 0 & \cdots & 2 \rho & -1 \\
0 & 0 & 0 & 0 & \cdots & -1 & 0
\end{array}\right]
$$

Tabela 3.1: Expressões para $W_{g}(u)$ e $W_{g}^{\prime}(u)$ para algumas distribuições elípticas.

\begin{tabular}{rcc}
\hline Distribuição & $W_{g}(u)$ & $W_{g}^{\prime}(u)$ \\
\hline Normal & $-\frac{1}{2}$ & 0 \\
t-Student & $-\frac{\nu+n}{2(\nu+u)}$ & $\frac{\nu+n}{2(\nu+u)^{2}}$ \\
Exponencial potência & $-\frac{1}{2}(\lambda) u^{\lambda-1}$ & $-\frac{1}{2} \lambda(\lambda-1) u^{(\lambda-2)}$ \\
\hline
\end{tabular}




\subsubsection{Informação de Fisher}

Seja $-\left.\ddot{\mathbf{L}}_{\theta \theta}\right|_{\hat{\theta}}$ a matriz de informação de Fisher observada para $\boldsymbol{\theta}$. Para calcular $-\left.\ddot{\mathbf{L}}_{\theta \theta}\right|_{\hat{\theta}}$ é necessário expressar as quantidades a seguir, que estão calculadas no Apêndice B.

$$
\begin{aligned}
& \ddot{\mathbf{L}}_{\beta \beta}=\frac{2}{\phi} \mathbf{X}^{\top} \mathbf{L}^{\top} \mathbf{L}\left(W_{g}(u)+\frac{2 W_{g}^{\prime}(u)}{\phi} \mathbf{e} \mathbf{e}^{\top} \mathbf{L}^{\top} \mathbf{L}\right) \mathbf{X}, \\
& \ddot{\mathbf{L}}_{\beta \phi}=\frac{2}{\phi^{2}} \mathbf{X}^{\top} \mathbf{L}^{\top} \mathbf{L}(\mathbf{y}-\mathbf{X} \boldsymbol{\beta})\left\{W_{g}(u)+W_{g}^{\prime}(u) u\right\}, \\
& \ddot{\mathbf{L}}_{\beta \rho}=-\frac{2}{\phi} \mathbf{X}^{\top}\left\{W_{g}(u)+\frac{W_{g}^{\prime}(u)}{\phi} \mathbf{L}^{\top} \mathbf{L} \mathbf{e} \mathbf{e}^{\top}\right\} \frac{\partial \mathbf{L}^{\top} \mathbf{L}}{\partial \rho} \mathbf{e}, \\
& \ddot{\mathbf{L}}_{\phi \phi}=\frac{1}{\phi^{2}}\left\{u\left(2 W_{g}(u)+u W_{g}^{\prime}(u)\right)+\frac{T}{2}\right\}, \\
& \ddot{\mathbf{L}}_{\phi \rho}=-\frac{1}{\phi^{2}}\left(W_{g}(u)+u W_{g}^{\prime}(u)\right) \mathbf{e}^{\top} \frac{\partial \mathbf{L}^{\top} \mathbf{L}}{\partial \rho} \mathbf{e} \quad \mathrm{e} \\
& \ddot{\mathbf{L}}_{\rho \rho}=-\frac{1+\rho^{2}}{\left(1-\rho^{2}\right)^{2}}+\frac{W_{g}(u)}{\phi} \mathbf{e}^{\top} \frac{\partial^{2} \mathbf{L}^{\top} \mathbf{L}}{\partial \rho \partial \rho} \mathbf{e}+\frac{W_{g}^{\prime}(u)}{\phi^{2}}\left\{\mathbf{e}^{\top} \frac{\partial \mathbf{L}^{\top} \mathbf{L}}{\partial \rho} \mathbf{e}\right\}^{2} .
\end{aligned}
$$

A partir de resultados de matrizes, a inversa da matriz $\ddot{\mathbf{L}}_{\theta \theta}$ pode ser expressa por

$$
\ddot{\mathbf{L}}_{\theta \theta}^{-1}=\left[\begin{array}{cc}
\mathbf{T}^{-1}+\mathbf{T}^{-1} \mathbf{U} \mathbf{Q}^{-1} \mathbf{U}^{\top} \mathbf{T}^{-1} & -\mathbf{T}^{-1} \mathbf{U} \mathbf{Q}^{-1} \\
-\mathbf{Q}^{-1} \mathbf{U}^{\top} \mathbf{T}^{-1} & \mathbf{Q}^{-1}
\end{array}\right]
$$


em que

$$
\begin{aligned}
\mathbf{T} & =\ddot{\mathbf{L}}_{\beta \beta}, \\
\mathbf{U} & =\left[\begin{array}{ll}
\ddot{\mathbf{L}}_{\beta \phi} & \ddot{\mathbf{L}}_{\beta \rho}
\end{array}\right], \\
\mathbf{Q} & =\mathbf{W}-\mathbf{U}^{\top} \mathbf{T}^{-1} \mathbf{U} \quad \mathrm{e} \\
\mathbf{W} & =\left[\begin{array}{ll}
\ddot{\mathbf{L}}_{\phi \phi} & \ddot{\mathrm{L}}_{\phi \rho} \\
\ddot{\mathrm{L}}_{\rho \phi} & \ddot{\mathrm{L}}_{\rho \rho}
\end{array}\right] .
\end{aligned}
$$

A matriz de informação esperada de Fisher para $\boldsymbol{\theta}$ pode ser expressa na forma

$$
\mathbf{K}_{\theta \theta}=\left[\begin{array}{ccc}
\mathbf{K}_{\beta \beta} & 0 & 0 \\
0 & \mathrm{~K}_{\phi \phi} & \mathbf{K}_{\phi \rho} \\
0 & \mathrm{~K}_{\rho \phi} & \mathrm{K}_{\rho \rho}
\end{array}\right]
$$

em que

$$
\begin{aligned}
\mathbf{K}_{\beta \beta}= & \frac{4 d_{g}}{T} \mathbf{X}^{\top} \frac{1}{\phi} \mathbf{L}^{\top} \mathbf{L} \mathbf{X}, \\
\mathrm{K}_{\phi \phi}= & \frac{1}{\phi^{2}}\left\{f_{g}-\frac{T^{2}}{4}\right\} \\
\mathrm{K}_{\phi \rho}= & \frac{T}{4 \phi}\left\{\frac{4 f_{g}}{T(T+2)}-1\right\} \operatorname{tr}\left(\mathbf{L}^{\top} \mathbf{L} \frac{\partial \mathbf{V}}{\partial \rho}\right)+ \\
& \frac{2 f_{g}}{T(T+2)} \operatorname{tr}\left(\frac{1}{\phi} \mathbf{L}^{\top} \mathbf{L} \frac{\partial \mathbf{V}}{\partial \rho}\right)
\end{aligned}
$$

$\mathrm{e}$

$$
\begin{aligned}
\mathrm{K}_{\rho \rho}= & \frac{1}{4}\left\{\frac{4 f_{g}}{T(T+2)}-1\right\}\left\{\operatorname{tr}\left(\mathbf{L}^{\top} \mathbf{L} \frac{\partial \mathbf{V}}{\partial \rho}\right)\right\}^{2}+ \\
& \frac{2 f_{g}}{T(T+2)} \operatorname{tr}\left(\left\{\mathbf{L}^{\top} \mathbf{L} \frac{\partial \mathbf{V}}{\partial \rho}\right\}^{2}\right),
\end{aligned}
$$


em que $\operatorname{tr}(\cdot)$ denota o traço de uma matriz, $d_{g}=E\left[W_{g}^{2}(U) U\right]$ e $f_{g}=$ $E\left[W_{g}^{2}(U) U^{2}\right] \operatorname{com} U=\left\|\mathbf{Z}^{2}\right\|, \mathbf{Z} \sim E C_{n}(\mathbf{0}, \mathbf{I}, \psi)$. A Tabela 3.2 mostra as expressões de $d_{g}$ e $f_{g}$ para algumas distribuições.

Como as inferências para o modelo AR(1) elíptico são similares às inferências para o modelo $A R(1)$ normal é razoável esperar que $\widehat{\boldsymbol{\beta}}$ é um estimador consistente e

$$
\sqrt{T}(\widehat{\boldsymbol{\beta}}-\boldsymbol{\beta}) \stackrel{d}{\rightarrow} N_{p}\left(\mathbf{0}, \mathbf{J}_{\beta \beta}^{-1}\right),
$$

em que $\mathbf{J}_{\beta \beta}=\lim _{T \rightarrow \infty} \frac{\mathbf{K}_{\beta \beta}}{T}$. Então, $\widehat{\mathbf{K}}_{\beta \beta}^{-1}=\frac{T \hat{\phi}}{4 d g}\left(\mathbf{X}^{\top} \mathbf{L}^{\top} \mathbf{L} \mathbf{X}\right)^{-1}$ é um estimador consistente da matriz de variância-covariância assintótica de $\widehat{\boldsymbol{\beta}}$. De modo análogo, espera-se que o estimador de máxima verossimilhança de $\boldsymbol{\alpha}=(\phi, \rho)^{\top}$ seja consistente e

$$
\sqrt{T}(\widehat{\boldsymbol{\alpha}}-\boldsymbol{\alpha}) \stackrel{d}{\rightarrow} N_{2}\left(\mathbf{0}, \mathrm{J}_{\alpha \alpha}^{-1}\right),
$$

em que $\mathrm{J}_{\alpha \alpha}=\lim _{n \rightarrow \infty} \frac{\mathrm{K}_{\alpha \alpha}}{n}$. Então,

$$
\widehat{\mathbf{K}}_{\alpha \alpha}^{-1}=\left[\begin{array}{cc}
\widehat{\mathrm{K}}_{\phi \phi} & \widehat{\mathrm{K}}_{\phi \rho} \\
\widehat{\mathrm{K}}_{\rho \phi} & \widehat{\mathrm{K}}_{\rho \rho}
\end{array}\right]^{-1}
$$

é um estimador consistente da variância assintótica de $\widehat{\boldsymbol{\alpha}}$.

Tabela 3.2: Valores de $d_{g}$ e $f_{g}$ para algumas distribuições elípticas.

\begin{tabular}{rcc}
\hline Distribuição & $d_{g}$ & $f_{g}$ \\
\hline Normal & $\frac{n}{4}$ & $\frac{n(n+2)}{4}$ \\
t-Student & $\frac{n}{4} \frac{\nu+n}{(\nu+n+2)}$ & $\frac{n(n+2)}{4} \frac{(\nu+n)}{(\nu+n+2)}$ \\
Exponencial potência & $\frac{\lambda^{2}}{2^{1 / \lambda}} \frac{\Gamma\left(\frac{n+2}{2 \lambda}+2\right)}{\Gamma\left(\frac{n}{2 \lambda}\right)}$ & $\frac{n(n+2 \lambda)}{4}$ \\
\hline
\end{tabular}




\subsection{Estimação}

A partir de (3.15) - (3.17) os estimadores de máxima verossimilhança para $\boldsymbol{\beta}$ e $\phi$ são dados por

$$
\begin{aligned}
\hat{\boldsymbol{\beta}}_{\mathrm{MV}} & =\left\{\mathbf{X}^{\top} \hat{\mathbf{L}}^{\top} \hat{\mathbf{L}} \mathbf{X}\right\}^{-1} \mathbf{X}^{\top} \hat{\mathbf{L}}^{\top} \hat{\mathbf{L}} \mathbf{y} \\
\mathrm{e} & \\
\hat{\phi_{\mathrm{MV}}} & =\frac{Q_{\hat{v}}(\hat{\boldsymbol{\beta}})}{T} .
\end{aligned}
$$

No caso de $\rho$, o estimador de máxima verossimilhança $\hat{\rho}_{\mathrm{MV}}$ satisfaz a equação

$$
\frac{\hat{\rho}_{\mathrm{MV}}}{1-\hat{\rho}_{\mathrm{MV}}^{2}}=\frac{W_{g}(\hat{u})}{\hat{\phi}_{\mathrm{MV}}}(\mathbf{y}-\mathbf{X} \hat{\boldsymbol{\beta}})^{\top} \frac{\widehat{\partial \mathbf{L}^{\top} \mathbf{L}}}{\partial \rho}(\mathbf{y}-\mathbf{X} \hat{\boldsymbol{\beta}}) .
$$

Conseqüentemente, o estimador de $\boldsymbol{\beta}$ pode ser obtido de um procedimento de mínimos quadrados reponderado por $\mathbf{L}^{\top} \mathbf{L}$. O estimador $\hat{\phi}_{\mathrm{MV}}$ leva em consideração tanto o peso $v$ quanto a autocorrelação $\rho$, através de $\mathbf{L}^{\top} \mathbf{L}$. Para se obter uma estimativa de $\rho$ é necessário resolver a equação (3.31), que está associada a distribuição dos erros através de $W_{g}(u)$. A solução de (3.31) pode levar a valores não admissíveis para $\hat{\rho}_{\mathrm{MV}}$ e procedimentos como Cochrane-Orcutt não levam em consideração a distribuição dos erros (vide, por exemplo, Harvey e Phillips; 1979, Seber e Wild; 1989, Zind-Walsh e Galbraith; 1991). Para contornar esses problemas optou-se pelo procedimento descrito a seguir. 


\section{Estimação iterada em dois estágios}

Para o primeiro estágio obtém-se as estimativas (3.29) e (3.30). Um chute inicial para $\hat{\rho}_{\text {MV }}$ pode ser dado por

$$
\hat{\rho}_{\mathrm{MV}}=\frac{\sum_{i=2}^{n} \hat{\mathrm{e}}_{i} \hat{\mathrm{e}}_{i-1}}{\sum_{i=1}^{n} \hat{\mathrm{e}}_{i}^{2}},
$$

que é a autocorrelação amostral de ordem um.

No segundo estágio escolhe-se $\hat{\rho}$ que maximiza o logaritmo da função de verossimilhança, para $\hat{\boldsymbol{\beta}}$ e $\hat{\phi}$ fixos. Para obter-se $\hat{\rho}$ no intervalo $(-1,1)$ deve-se usar um método de maximização com barreira. $\mathrm{O}$ algoritmo pode ser resumido da seguinte forma:

\section{Estágio 1:}

$$
\begin{aligned}
& \boldsymbol{\beta}^{(m+1)}=\left\{\mathbf{X}^{\top}\left(\mathbf{L}^{(m)}\right)^{\top} \mathbf{L}^{(m)} \mathbf{X}\right\}^{-1} \mathbf{X}^{\top}\left(\mathbf{L}^{(m)}\right)^{\top} \mathbf{L}^{(m)} \mathbf{y} \\
& \phi^{(m+1)}=\frac{Q_{v^{(m+1)}}\left(\boldsymbol{\beta}^{(m+1)}\right)}{T} .
\end{aligned}
$$

\section{Estágio 2:}

$$
\rho^{(m+1)}=\operatorname{argmax}_{\rho}\left\{\mathrm{L}\left(\boldsymbol{\beta}^{(m+1)}, \phi^{(m+1)}, \rho\right)\right\},
$$

para $m=0,1,2, \ldots$ e a quantidade $Q_{v}(\boldsymbol{\beta})$ está definida a partir da fórmula (3.16).

\subsection{Exemplo}

\subsubsection{Modelo CAPM}

O modelo (3.2) - (3.5) pode ser usado para introduzir uma estrutura de correlação no modelo CAPM. A introdução dessa estrutura 
de erro justifica-se porque os dados utilizados pelo CAPM constituem uma série temporal e, pela teoria das finanças, não há uma dependência "longa" entre as observações. Além disso, espera-se uma baixa correlação para esses dados. Segue-se que o modelo CAPM com estrutura de erro $A R(1)$, pode ser adequado, sendo dado por:

$$
\begin{aligned}
R_{t}-r_{f t} & =\alpha+\beta\left(R_{M t}-r_{f t}\right)+\epsilon_{t} \mathrm{e} \\
\epsilon_{t} & =\rho \epsilon_{t-1}+\delta_{t},
\end{aligned}
$$

em que $R_{t}$ é o retorno de um ativo entre os períodos de tempo $t \mathrm{e}$ $(t-1), R_{M t}$ é o retorno de um índice de mercado entre os períodos $t$ e $(t-1), r_{f t}$ denota a taxa de retorno entre os períodos $t$ e $(t-1)$ de um ativo livre de risco e $\epsilon_{t}$ representa o erro aleatório, descrito como um processo $\mathrm{AR}(1)$ pela equação (3.37), em que $\delta_{t}$ representa o choque aleatório.

A regressão (3.36) - (3.37) pode ser redefinida como

$$
\begin{aligned}
y_{t} & =\alpha+\beta x_{t}+\epsilon_{t} \mathrm{e} \\
\epsilon_{t} & =\rho \epsilon_{t-1}+\delta_{t},
\end{aligned}
$$

em que $y_{t}=R_{t}-r_{f t}$ e $x_{t}=R_{M t}-r_{f t}$, que são os excessos de retornos, e as quantidades $\delta_{t}$ e $\epsilon_{t}$ definidas anteriormente.

As estimativas dos parâmetros da regressão (3.36) - (3.37) para o Banco Itaú baseadas na suposição de normalidade para o choque aleatório $\delta_{t}$ são apresentadas a seguir e uma análise de diagnóstico para esse modelo está no Capítulo 4. Os dados usados são de janeiro de 1998 a agosto de 2004. Adotou-se o Ibovespa como proxy do mercado e o CDI 30 nominal como taxa livre de risco.

A Figura 3.1 mostra os retornos mensais do Itaú e do Ibovespa. Os retornos parecem estacionários e ergóticos e tendem a se mover na mesma direção ao longo do tempo. 

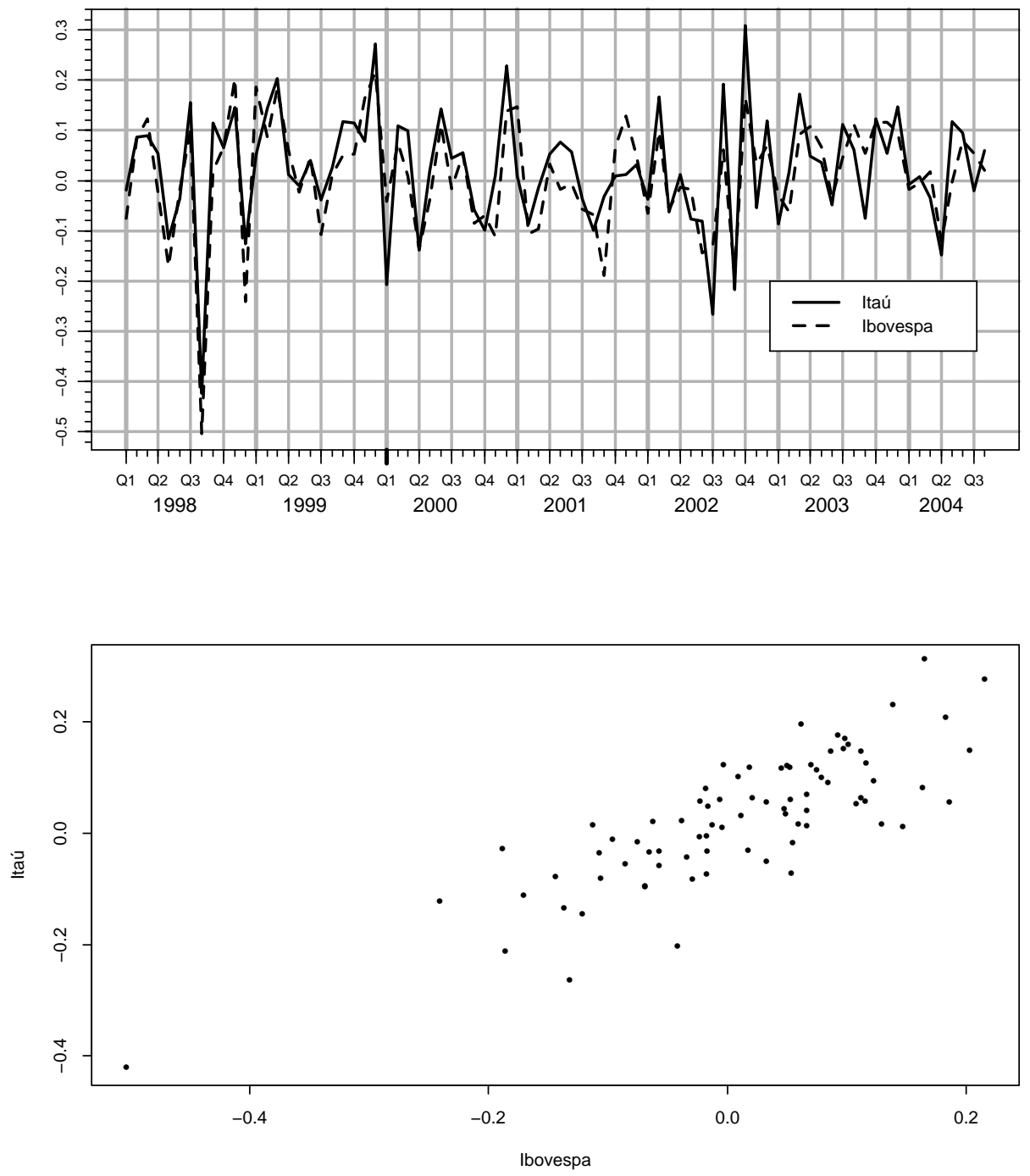

Figura 3.1: Retornos mensais do Itaú e do Ibovespa. 
Tabela 3.3: Estimativas dos parâmetros do modelo (3.36) - (3.37) para o Banco Itaú, supondo distribuição normal para os choques aleatórios $\delta$ 's.

\begin{tabular}{crrrr}
\hline Coeficiente & Valor & Erro padrão & Z-valor & P-valor \\
\hline$\alpha$ & $-0,0399$ & 0,0405 & $-0,9845$ & 0,3249 \\
$\beta$ & 0,9547 & 0,0345 & 27,6427 & $<0,0001$ \\
$\rho$ & $-0,2873$ & 0,1076 & & \\
$\phi$ & 0,0044 & 0,0007 & & \\
\hline
\end{tabular}

Os parâmetros estimados para o modelo CAPM do Itaú usando a suposição de erro $\mathrm{AR}(1)$ e choques aleatórios $\delta$ com distribuição normal são dados pela Tabela 3.3. Como o parâmetro $\alpha$ apresenta o p-valor igual a 0,3249, a regressão foi reestimada, considerando $\alpha$ igual a zero. As estimativas são dadas pela Tabela 3.4.

Tabela 3.4: Estimativas dos parâmetros do modelo (3.36) - (3.37) para o Banco Itaú, supondo distribuição normal para os choques aleatórios $\delta$ 's.

\begin{tabular}{crrrr}
\hline Coeficiente & Valor & Erro padrão & Z-valor & P-valor \\
\hline$\beta$ & 0,9883 & 0,0050 & 198,8838 & $<0,0001$ \\
$\rho$ & $-0,2833$ & 0,1078 & & \\
$\phi$ & 0,0045 & 0,0007 & & \\
\hline
\end{tabular}

A estimativa para $\beta$ do Itaú é 0,9883 com um erro padrão estimado igual a 0,0050. Um intervalo com $95 \%$ de confiança para $\beta$ é $(0,9786 ; 0,9981)$. Ou seja, o Itaú pode ser julgado como sendo um papel conservador, em relação ao Ibovespa. A estimativa para o coeficiente de correlação $\rho$ é -0,2873, com erro padrão igual a 0, 1076 e o $\phi$ estimado é 0,0044, com erro padrão igual a 0,0007. A magnitude de risco diversificável estimada é dada por $\sqrt{\hat{\phi}}=0,0668$ ou $6,7 \%$ por mês.

Para ilustrar o fato que os estimadores dos coeficientes da regressão são não viesados mas não têm variância mínima, quando não se inclui o parâmetro $\rho$, a Tabela 3.5 apresenta a estimativa de $\beta$ sem considerar a autocorrelação. Nesse caso, um intervalo com $95 \%$ de confiança para $\beta$ é $(0,9749 ; 1,0010)$. Portanto, diferentemente da decisão baseada no modelo (3.36) - (3.37), não se pode rejeitar a hipótese que beta é igual a um. Dessa forma, este exemplo ilustra a importância da 
metodologia, mesmo para uma baixa correlação, como é o caso, dada pela estimativa $\hat{\rho}=-0,2826$.

Tabela 3.5: Estimativas dos parâmetros do modelo CAPM para o Banco Itaú, supondo distribuição normal com erros independentes.

\begin{tabular}{crrrr}
\hline Coeficiente & Valor & Erro padrão & Z-valor & P-valor \\
\hline$\beta$ & 0,9880 & 0,0067 & 149,1630 & $<0,0001$ \\
$\phi$ & 0,0049 & 0,0008 & & \\
\hline
\end{tabular}

A Figura 3.2 mostra o diagrama de dispersão entre os excessos de retornos do Itaú e do Ibovespa, a reta ajustada para o modelo normal linear com erros $\mathrm{AR}(1)$ e a reta ajustada para o modelo normal linear com erros independentes. Constata-se que as retas estimadas praticamente coincidem, fato esperado pois os estimadores de $\boldsymbol{\beta}$ são não viesados para os dois modelos considerados.

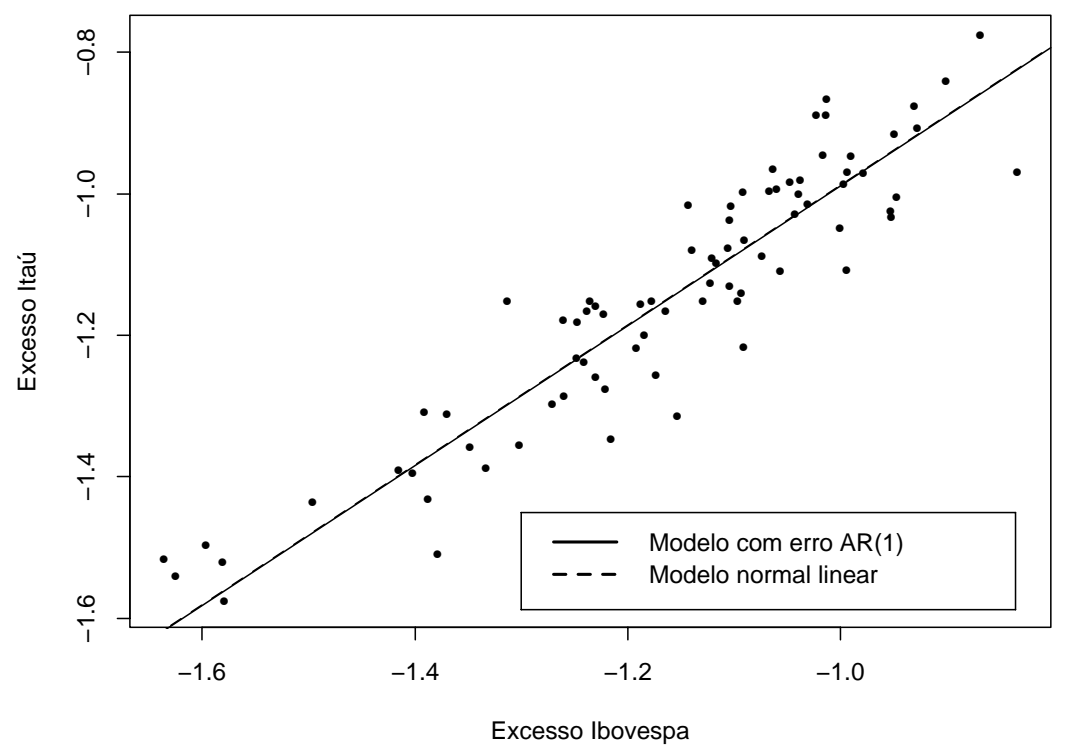

Figura 3.2: Reta ajustada pelos modelos de regressão autoregressivo e normal linear e gráfico de dispersão entre os excessos de retorno do Ibovespa e Itaú. 
Capítulo 4

\section{Métodos de diagnóstico em modelos simétricos de regressão linear com erros $\operatorname{AR}(1)$}

Introdução

Quando um modelo de regressão é considerado para uma aplicação, normalmente não se tem certeza que o mesmo é apropriado. Algumas, ou muitas, das características do modelo, como a linearidade da função de regressão ou a distribuição adotada para o erro, podem não ser apropriadas para o conjunto de dados em estudo e, portanto, conclusões baseadas nesse modelo podem estar erradas. Então, é importante examinar o quanto o modelo que está sendo considerado é adequado para os dados antes de fazer inferências. Esta avaliação do modelo é conhecida como diagnóstico, que consiste na verificação de possíveis afastamentos relevantes das suposições feitas para o mesmo, bem como na verificação da existência de observações com alguma in- 
terferência desproporcional nos resultados do ajuste. Os métodos de análise de diagnóstico tem longa data e iniciaram-se com o estudo de resíduos (vide, por exemplo, Paula, 2004). Kim e Huggins (1998) apresentaram esquema de diagnóstico para modelos de regressão com estrutura de erro gaussiano autocorrelacionado.

A análise de resíduos é importante para detectar a presença de pontos extremos ou outliers, que são observações mal ajustadas, e avaliar a adequabilidade da distribuição proposta para o erro. Uma referência importante nesse tópico é o artigo de Cox e Snell (1968). Observações extremas geralmente têm um efeito desproporcional no ajuste da função de regressão. Portanto, é importante estudá-las cuidadosamente para decidir se elas devem ser excluídas ou permanecerem, e se permanecerem, a influência dessas observações deve ser reduzida no processo de ajuste e/ou o modelo de regressão deve ser revisado. Uma forma de reduzir a influência dessas observações é adotar para o erro distribuições com caudas mais pesadas do que a distribuição normal (vide, por exemplo, Cysneiros, 2004). Com relação à distribuição proposta para o erro, pode-se avaliar, a partir dos resíduos:

- homocedasticidade;

- linearidade da função de regressão;

- independência dos erros;

- normalidade dos erros;

- ausência de uma ou mais variáveis preditoras.

Atkinson (1985) e Cook, e Weisberg (1983) apresentam, sob erros normais, métodos gráficos para detectar heteroscedasticidade.

Também é importante avaliar pontos que apresentam um peso desproporcional no próprio valor ajustado, os pontos de alavanca. Esses pontos em geral são remotos no subespaço gerado pelas colunas da matriz modelo $\mathbf{X}$, ou seja têm um perfil diferente dos demais pontos no que diz respeito aos valores das variáveis explicativas (vide, por exemplo, Paula, 2004). Dependendo da localização, os pontos de alavanca 
podem exercer forte influência nas estimativas dos coeficientes da regressão. Wei, Hu e Fung (1998) generalizaram a definição de pontos de alavanca para modelos bastante gerais cuja variável resposta seja contínua.

Outro tópico importante na análise de diagnóstico é a detecção de observações influentes, isto é, pontos que exercem um peso desproporcional nas estimativas dos parâmetros do modelo. Cook (1986) propõe avaliar a influência de pontos através da medida denominada influência local. Essa medida de influência é especialmente apropriada para avaliar o modelo descrito no Capítulo 3 por não ser baseada na deleção de observações, como é o caso de outras medidas de influência, por exemplo a Distância de Cook.

Este capítulo apresenta métodos de diagnóstico para os modelos de regressão discutidos no Capítulo 3. Nas Seções $4.1-4.3$ são definidos, respectivamente, resíduos, pontos de alavanca e duas medidas de influência local. Para exemplificar a metodologia apresentada, exibe-se uma análise de diagnóstico para o exemplo apresentado no Capítulo 3.

\subsection{Resíduos}

O resíduo para uma observação pode ser definido como uma função do tipo $r_{i}=r\left(y_{i}, \hat{\mu}_{i}\right)$ que procura medir a discrepância entre o valor observado e o valor ajustado. Em particular, o resíduo $r_{i}=y_{i}-\hat{\mu}_{i}$, denominado resíduo ordinário, é a definição mais freqüente (vide, por exemplo, Paula; 2004 e Haslett, e Kevin; 1998).

Para o modelo de regressão definido por (2.2) e (2.3), Cysneiros (2004) concluiu que o vetor de resíduos ordinários

$$
\mathbf{r}=\mathbf{y}-\mathbf{X} \hat{\boldsymbol{\beta}}
$$

em que os elementos do vetor $\mathbf{y}$ são dados por $y_{i}=\mathbf{x}_{i}^{\top} \boldsymbol{\beta}+\epsilon_{i}$, tem aproximadamente

$$
\mathrm{E}[\mathbf{r}]=\mathbf{0}
$$


e

$$
\operatorname{Var}[\mathbf{r}]=\xi \phi\left\{\mathbf{I}_{n}-\left(4 d_{g} \xi\right)^{-1}\right\} \mathbf{H},
$$

em que $\mathbf{H}=\mathbf{X}\left(\mathbf{X}^{\top} \mathbf{X}\right)^{-1} \mathbf{X}^{\top}, \mathbf{X}$ é a matriz modelo, sendo $\mathbf{H}$ conhecida como matriz hat, e $d_{g}=E\left[W_{g}^{2}(U) U\right]$ com $U \sim S(1)$.

Como os $r_{i}^{\prime} s$ têm variâncias diferentes, é conveniente expressálos de forma padronizada a fim de permitir a comparabilidade entre os mesmos. O modo de se padronizar o resíduo $r_{i}$ é subtraí-lo de sua média em seguida dividir por seu desvio padrão (vide, por exemplo, Paula, 2004). Portanto, os resíduos padronizados ficam expressos por:

$$
t_{r_{i}}=\frac{y_{i}-\hat{y}_{i}}{\{\xi \hat{\phi}\}^{1 / 2}\left\{1-\left(4 d_{g} \xi\right)^{-1} \hat{h_{i i}}\right\}^{1 / 2}} .
$$

Para o modelo definido por (3.2) - (3.5), o vetor de resíduos ordinários (4.1) tem esperança zero e variância

$$
\operatorname{Var}[\mathbf{r}]=\xi \phi\left\{\mathbf{V}-\mathbf{X}\left(\mathbf{X}^{\top} \mathbf{L}^{\top} \mathbf{L} \mathbf{X}\right)^{-1} \mathbf{X}^{\top}\right\}
$$

em que as matrizes $\mathbf{V}$ e $\mathbf{L}$ estão definidas na Seção 3.2.1. Os resíduos padronizados são obtidos de modo análogo aos caso anterior, em que o desvio padrão é dado pelo elemento $(i, i)$ da matriz de variânciacovariância (4.3). No entanto, os resíduos padronizados dessa maneira são correlacionados, não refletindo o comportamento do choque aleatório $\delta$, no modelo (3.2) - (3.5). Para contornar este problema, foi proposto o resíduo definido por:

$$
r_{t}=y_{t}-x_{t}^{\top} \widehat{\boldsymbol{\beta}}-\hat{\rho} \hat{\epsilon}_{t-1} .
$$

Esse resíduo é aproximadamente não correlacionado e tem variância aproximadamente $\xi \phi$. Deste modo,

$$
t_{r_{t}}=\frac{y_{t}-x_{t}^{\top} \widehat{\boldsymbol{\beta}}-\hat{\rho} \hat{\epsilon}_{t-1}}{\sqrt{\xi \hat{\phi}}} .
$$

é o resíduo padronizado proposto para o modelo (3.2) - (3.5). Observase da definição $(3.2)-(3.5)$ que $r_{t}=\hat{\delta}_{t}$. 
4.2 Pontos de alavanca

A principal idéia por trás do conceito de ponto de alavanca (vide, por exemplo, Hoaglin e Welsch, (1978); Cook e Weisberg, (1982); Emerson, Hoaglin e Kempthorne, (1984); St. Laurent e Cook (1992) e Wei, Hu e Fung, (1998)) é conhecer a influência de $y_{i}$ no próprio valor predito. Essa influência pode ser bem representada pela derivada

$$
\frac{\partial \widehat{y}_{i}}{\partial y_{i}}
$$

que, no caso do modelo definido por (2.2) e (2.3) com distribuição normal (modelo normal linear), é igual a $h_{i i}$, em que $h_{i i}$ é o $i$-ésimo elemento da diagonal principal da matriz de projeção $\mathbf{H}=\mathbf{X}\left(\mathbf{X}^{\top} \mathbf{X}\right)^{-1} \mathbf{X}^{\top}$ e $\mathbf{X}$ é a matriz modelo. Supondo que todos os pontos exerçam a mesma influência sobre os valores ajustados, espera-se que $h_{i i}$ esteja próximo de $\operatorname{tr}(\mathbf{H}) / n$. Para maiores detalhes sobre as propriedades da matriz $\mathbf{H}$ Cook e Weisberg (1982); Atkinson (1985) e Chatterjee e Hadi (1988).

A matriz generalizada de pontos de alavanca (vide Wei, Hu e Fung (1998))

$$
\frac{\partial \widehat{\mathbf{y}}}{\partial \mathbf{y}}
$$

pode ser expressa na forma

$$
\mathbf{G L}(\widehat{\boldsymbol{\theta}})=\left.\left\{\mathbf{D}_{\theta}\left(-\ddot{\mathbf{L}}_{\theta \theta}\right)^{-1} \ddot{\mathbf{L}}_{\theta \mathbf{y}}\right\}\right|_{\boldsymbol{\theta}=\widehat{\boldsymbol{\theta}}},
$$

em que

$$
\begin{aligned}
\mathbf{D}_{\theta} & =\partial \boldsymbol{\mu} / \partial \boldsymbol{\theta}^{\top}, \\
\ddot{\mathbf{L}}_{\theta \theta} & =\partial^{2} \mathrm{~L}(\boldsymbol{\theta} ; \mathbf{y}) / \partial \boldsymbol{\theta} \partial \boldsymbol{\theta}^{\top} \quad \mathrm{e} \\
\ddot{\mathbf{L}}_{\theta \mathbf{y}} & =\partial^{2} \mathrm{~L}(\boldsymbol{\theta} ; \mathbf{y}) / \partial \boldsymbol{\theta} \partial \mathbf{y}^{\top} .
\end{aligned}
$$

A expressão (4.6) generaliza a definição de pontos de alavanca dada em St. Laurent e Cook (1992).

Cysneiros (2004) mostra que para o modelo simétrico de regressão linear, definido por (2.2) e (2.3), as matrizes $\mathbf{D}_{\theta}$ e $\ddot{\mathbf{L}}_{\theta \mathbf{y}}$ são dadas por

$$
\mathbf{D}_{\theta}=(\mathbf{X}, \mathbf{0})
$$


e

$$
\ddot{\mathbf{L}}_{\theta \mathbf{y}}=\left[\begin{array}{c}
\ddot{\mathbf{L}}_{\beta \mathbf{y}} \\
\ddot{\mathbf{L}}_{\phi \mathbf{y}}
\end{array}\right]=\left[\begin{array}{c}
\frac{1}{\phi} \mathbf{X D}(\mathbf{a}) \\
-\frac{2}{\phi^{2}} \mathbf{b}^{\top}
\end{array}\right],
$$

em que $\mathbf{D}(\mathbf{a})=\operatorname{diag}\left\{a_{1}, a_{2}, \ldots, a_{n}\right\}, a_{i}=\left\{v_{i}-4 W_{g}^{\prime}\left(u_{i}\right) u_{i}\right\}, \mathbf{b}=\left(b_{1}\right.$, $\left.b_{2}, \ldots, b_{n}\right)^{\top}, b_{i}=\left\{W_{g}\left(u_{i}\right)+u_{i} W_{g}^{\prime}\left(u_{i}\right)\right\} e_{i}$ com $i=1,2, \ldots, n$. Desse modo, a matriz generalizada de pontos de alavanca (4.6) assume a forma simplificada

$$
\mathbf{G L}(\widehat{\boldsymbol{\theta}})=\widehat{\mathbf{H}}+\frac{4}{\hat{\mathrm{E}} \hat{\phi}} \widehat{\mathbf{H}} \mathbf{D}^{-1}(\hat{\mathbf{a}}) \hat{\mathbf{b}} \hat{\mathbf{b}}^{\top}\left\{\mathbf{I}_{n}-\widehat{\mathbf{H}}\right\}
$$

em que $\widehat{\mathbf{H}}=\mathbf{X}\left(\mathbf{X}^{\top} \mathbf{D}(\hat{\mathbf{a}}) \mathbf{X}\right)^{-1} \mathbf{X}^{\top} \mathbf{D}(\hat{\mathbf{a}}), \mathbf{I}_{n}$ é a matriz identidade de ordem $n$ e a quantidade $\hat{E}$ está definida na Seção 2.2 .

Para o modelo (3.2) - (3.5), de acordo com os resultados do Apêndice $\mathrm{C}$, as matrizes $\mathbf{D}_{\theta}$ e $\ddot{\mathbf{L}}_{\theta \mathbf{y}}$ são dadas por

$$
\mathbf{D}_{\theta}=(\mathbf{X}, \mathbf{0}, \mathbf{0})
$$

e

$$
\ddot{\mathbf{L}}_{\theta \mathbf{y}}=\left[\begin{array}{c}
\ddot{\mathbf{L}}_{\beta \mathbf{y}} \\
\ddot{\mathbf{L}}_{\phi \mathbf{y}} \\
\ddot{\mathbf{L}}_{\rho \mathbf{y}}
\end{array}\right]
$$

em que

$$
\begin{aligned}
& \ddot{\mathbf{L}}_{\beta \mathbf{y}}=-\frac{2}{\phi} \mathbf{X}^{\top} \mathbf{L}^{\top} \mathbf{L}\left\{W_{g}(u)+2 \frac{W_{g}^{\prime}(u)}{\phi} \mathbf{e}^{\top} \mathbf{e}^{\top} \mathbf{L}^{\top} \mathbf{L}\right\} \\
& \ddot{\mathbf{L}}_{\phi \mathbf{y}}=-\frac{2}{\phi^{2}}\left\{W_{g}(u)+u W_{g}^{\prime}(u)\right\} \mathbf{e}^{\top} \mathbf{L}^{\top} \mathbf{L} \mathrm{e} \\
& \ddot{\mathbf{L}}_{\rho \mathbf{y}}=\frac{2}{\phi} \mathbf{e}^{\top}\left\{W_{g}(u) \frac{\partial \mathbf{L}^{\top} \mathbf{L}}{\partial \rho}+\frac{W_{g}^{\prime}(u)}{\phi} \mathbf{e}^{\top} \frac{\partial \mathbf{L}^{\top} \mathbf{L}}{\partial \rho} \mathbf{e} \mathbf{L}^{\top} \mathbf{L}\right\},
\end{aligned}
$$

para $\mathbf{e}=(\mathbf{y}-\mathbf{X} \boldsymbol{\beta})$. Apesar de não se obter uma forma simplificada, os resultados podem ser implementados facilmente. 


\subsection{Influência local}

Influência local tem por objetivo avaliar, através de uma medida apropriada de influência, o efeito de pequenas perturbações no modelo ou nos dados, essas perturbações são definidas convenientemente. Há indícios de que o modelo está mal ajustado ou que as suposições feitas não são apropriadas quando as perturbações causam efeitos desproporcionais em determinados resultados do modelo. A identificação das observações responsáveis por essas discrepâncias pode ajudar na escolha de um modelo mais adequado aos dados.

A medida de influência mais conhecida é o afastamento da verossimilhança

$$
\mathrm{LD}(\boldsymbol{\omega})=2\left\{\mathrm{~L}(\widehat{\boldsymbol{\theta}})-\mathrm{L}\left(\widehat{\boldsymbol{\theta}}_{\omega}\right)\right\}
$$

em que $\widehat{\boldsymbol{\theta}}_{\omega}$ denota a estimativa de máxima verossimilhança sob o modelo perturbado e $\boldsymbol{\omega}=\left(\omega_{1}, \omega_{2}, \ldots, \omega_{s}\right)^{\top}$ é o vetor de perturbações aplicadas ao modelo. A proposta de Cook (1986) é estudar o comportamento de $\operatorname{LD}(\boldsymbol{\omega})$, ou alguma outra medida de influência, em torno do vetor de não-perturbação $\boldsymbol{\omega}_{0}$, em que $\operatorname{LD}\left(\boldsymbol{\omega}_{0}\right)=0$. Logo, $\boldsymbol{\omega}_{0}$ é um ponto de mínimo da função $\operatorname{LD}(\boldsymbol{\omega})$, pois $\operatorname{LD}(\boldsymbol{\omega}) \geq 0$. A sugestão de Cook (1986) é investigar a curvatura normal da linha projetada $\operatorname{LD}\left(\boldsymbol{\omega}_{0}+a \boldsymbol{\ell}\right)$, em que $a \in \mathbb{R}$, em torno de $a=0$ para alguma direção arbitrária $\ell,\|\ell\|=1$. Mostra-se que a curvatura normal pode ser expressa por

$$
C_{l}(\boldsymbol{\theta})=2\left|\boldsymbol{\ell}^{\top} \boldsymbol{\Delta}^{\top} \ddot{\mathbf{L}}_{\theta \theta}^{-1} \boldsymbol{\Delta} \boldsymbol{\ell}\right|,
$$

em que $\boldsymbol{\Delta}$ é uma matriz $(p+q) \times s$ com elementos

$$
\Delta_{i j}=\partial^{2} \mathrm{~L}(\boldsymbol{\theta} \mid \boldsymbol{\omega}) / \partial \theta_{i} \partial \omega_{j}
$$

para $i=1,2, \ldots,(p+q)$ e $j=1,2, \ldots, s$, com todas as quantidades sendo avaliadas em $\boldsymbol{\omega}=\boldsymbol{\omega}_{0}$ e $\boldsymbol{\theta}=\widehat{\boldsymbol{\theta}}$. Toma-se a direção correspondente à maior curvatura, maior autovetor - denotado por $\boldsymbol{\ell}_{\text {max }}-$ é o maior autovalor da matriz $\mathbf{B}=-\boldsymbol{\Delta}^{\top} \ddot{\mathbf{L}}_{\theta \theta}^{-1} \boldsymbol{\Delta}$ - denotado por $C_{l_{\max }}$. O gráfico de índices de $\boldsymbol{\ell}_{\max }$ pode mostrar como se deve perturbar, por exemplo, o parâmetro de escala para obter maiores mudanças nas estimativas de $\boldsymbol{\theta}$. Contudo, se o interesse é somente no vetor $\boldsymbol{\beta}$, a curvatura normal na direção $\ell$ é dada por

$$
C_{l}(\boldsymbol{\beta})=2\left|\boldsymbol{\ell}^{\top} \boldsymbol{\Delta}^{\top}\left(\ddot{\mathbf{L}}_{\theta \theta}^{-1}-\mathbf{L}_{1}\right) \boldsymbol{\Delta} \boldsymbol{\ell}\right|,
$$


em que

$$
\mathbf{L}_{1}=\left[\begin{array}{cc}
0 & 0 \\
0 & \ddot{\mathbf{L}}_{\gamma \gamma}^{-1}
\end{array}\right]
$$

com $\ddot{\mathbf{L}}_{\gamma \gamma}^{-1}$ sendo a matriz de informação observada de Fisher para $\gamma$. O gráfico de índices do maior autovetor de $\boldsymbol{\Delta}^{\top}\left(\ddot{\mathbf{L}}_{\theta \theta}^{-1}-\mathbf{L}_{1}\right) \boldsymbol{\Delta}$ pode revelar quais são as observações influentes em $\hat{\boldsymbol{\beta}}$. Similarmente, a curvatura normal para os parâmetros de escala contidos no vetor $\gamma$ na direção $\ell$ é dada por

$$
C_{l}(\gamma)=2\left|\ell^{\top} \Delta^{\top}\left(\ddot{\mathbf{L}}_{\theta \theta}^{-1}-\mathbf{L}_{2}\right) \Delta \ell\right|
$$

em que

$$
\mathbf{L}_{2}=\left[\begin{array}{cc}
\ddot{\mathbf{L}}_{\beta \beta}^{-1} & 0 \\
0 & 0
\end{array}\right]
$$

com $\ddot{\mathbf{L}}_{\beta \beta}^{-1}$ sendo a matriz de informação observada de Fisher para $\boldsymbol{\beta}$. A influência local das observações em $\hat{\gamma}$ pode ser avaliada considerando-se o gráfico de índices de $\boldsymbol{\ell}_{\max }$ para a matriz $\boldsymbol{\Delta}^{\top}\left(\ddot{\mathbf{L}}_{\theta \theta}^{-1}-\mathbf{L}_{2}\right) \boldsymbol{\Delta}$.

Como $C_{l}(\boldsymbol{\theta})$ não é invariante a mudanças uniformes na escala Poon e Poon (1999) propuseram a curvatura conformal

$$
B_{l}(\boldsymbol{\theta})=C_{l}(\boldsymbol{\theta}) /\left\|2 \boldsymbol{\Delta}^{\top} \ddot{\mathbf{L}}_{\theta \theta}^{-1} \boldsymbol{\Delta}\right\|_{\mathrm{F}},
$$

em que $\|\cdot\|_{F}$ denota a norma Frobenius definida como

$$
\|\mathbf{A}\|_{\mathrm{F}}=\left\{\operatorname{tr}\left(\mathbf{A}^{\top} \mathbf{A}\right)\right\}^{1 / 2}
$$

com A sendo uma matrix $m \times n$. Uma propriedade interessante da curvatura conformal é que para qualquer direção unitária $\boldsymbol{\ell}$ tem-se que $0<B_{l}(\boldsymbol{\theta})<1$. Uma outra possibilidade é considerar a influência total local que consiste de gráficos de $C_{i}=C_{\ell_{i}} / \sum_{j=1}^{s} C_{\ell_{j}}$, em que $\boldsymbol{\ell}_{i}$ é um vetor de zeros com 1 na $i$-ésima posição (Lesaffre e Verbeke, 1998).

Dado que é possível perturbar o modelo proposto de diversas maneiras, é importante escolher perturbações que sejam de fácil interpretação. Galea, Paula e Bolfarine (1997) e Galea, Paula e Uribe-Opazo (2003) avaliaram a influência das observações nas estimativas dos parâmetros usando o enfoque de influência local na classe dos modelos 
simétricos lineares. Tsai e Wu (1992) e Liu (2000), (2002) e (2004) discutiram influência local em modelos elípticos. Paula, Cysneiros e Galea (2003) discutiram medidas de influência e alavanca em modelos elípticos não lineares. A seguir são apresentados dois esquemas de perturbação para o modelo (3.2) - (3.5).

4.3.1 Perturbação aditiva na resposta dos modelos simétricos de regressão com erros $\mathrm{AR}(1)$

Considere a perturbação aditiva na variável resposta do modelo $(3.2)-(3.5)$, isto é

$$
\mathbf{y}_{\omega}=\mathbf{y}+s \boldsymbol{\omega},
$$

em que $s$ é uma medida de escala. Segue-se que a densidade do modelo perturbado fica dada por

$$
f_{\mathbf{y}}(\mathbf{y} \mid \boldsymbol{\omega})=|\boldsymbol{\Sigma}|^{-1 / 2} g\left(u_{\omega}\right),
$$

em que

$$
u_{\omega}=(\mathbf{y}+s \boldsymbol{\omega}-\mathbf{X} \boldsymbol{\beta})^{\top} \boldsymbol{\Sigma}^{-1}(\mathbf{y}+s \boldsymbol{\omega}-\mathbf{X} \boldsymbol{\beta}) .
$$

A partir dos resultados exibidos no Apêndice D, mostra-se que a matriz $\boldsymbol{\Delta}$ para este esquema de perturbação fica dada por

$$
\boldsymbol{\Delta}=\left[\begin{array}{c}
\partial^{2} \mathrm{~L}(\boldsymbol{\theta} \mid \boldsymbol{\omega}) / \partial \boldsymbol{\beta} \partial \boldsymbol{\omega}^{\top} \mid \boldsymbol{\omega}_{0} \\
\partial^{2} \mathrm{~L}(\boldsymbol{\theta} \mid \boldsymbol{\omega}) / \partial \phi \partial \boldsymbol{\omega}^{\top} \mid \boldsymbol{\omega}_{0} \\
\partial^{2} \mathrm{~L}(\boldsymbol{\theta} \mid \boldsymbol{\omega}) / \partial \rho \partial \boldsymbol{\omega}^{\top} \mid \boldsymbol{\omega}_{0}
\end{array}\right]
$$

em que

$$
\begin{aligned}
\partial^{2} \mathrm{~L}(\boldsymbol{\theta} \mid \boldsymbol{\omega}) / \partial \boldsymbol{\beta} \partial \boldsymbol{\omega}^{\top} & =-\frac{2 s}{\phi} \mathbf{X}^{\top} \mathbf{L}^{\top} \mathbf{L}\left\{W_{g}\left(u_{\omega}\right)+\frac{2}{\phi} W_{g}^{\prime}\left(u_{\omega}\right) \mathbf{e}_{\omega} \mathbf{e}_{\omega}^{\top} \mathbf{L}^{\top} \mathbf{L}\right\} \\
\partial^{2} \mathrm{~L}(\boldsymbol{\theta} \mid \boldsymbol{\omega}) / \partial \phi \partial \boldsymbol{\omega}^{\top} & =-\frac{2 s}{\phi^{2}}\left\{W_{g}\left(u_{\omega}\right)+u W_{g}^{\prime}\left(u_{\omega}\right)\right\} \mathbf{e}_{\omega}^{\top} \mathbf{L}^{\top} \mathbf{L} \quad \mathrm{e} \\
\partial^{2} \mathrm{~L}(\boldsymbol{\theta} \mid \boldsymbol{\omega}) / \partial \rho \partial \boldsymbol{\omega}^{\top} & =\frac{2 s}{\phi} \mathbf{e}_{\omega}^{\top} \frac{\partial \mathbf{L}^{\top} \mathbf{L}}{\partial \rho}\left\{W_{g}\left(u_{\omega}\right)+\frac{W_{g}^{\prime}\left(u_{\omega}\right)}{\phi^{2}} \mathbf{e}_{\omega} \mathbf{e}_{\omega}^{\top} \mathbf{L}^{\top} \mathbf{L}\right\},
\end{aligned}
$$


com $\mathbf{e}_{\omega}=(\mathbf{y}+s \boldsymbol{\omega}-\mathbf{X} \boldsymbol{\beta})$ e $\boldsymbol{\Sigma}^{-1}=\frac{1}{\phi} \mathbf{L}^{\top} \mathbf{L}$. Estes resultados, assim como os de pontos de alavanca, podem ser facilmente implementados.

4.3.2 Perturbação aditiva na $i$-ésima variável explicativa dos modelos simétricos de regressão com erros $\mathrm{AR}(1)$

Considere a perturbação aditiva em uma variável explicativa contínua do modelo $(3.2)-(3.5)$, isto é

$$
\mathbf{x}_{i \omega}=\mathbf{x}_{i}+s \boldsymbol{\omega},
$$

em que $s$ é uma medida de escala. Tem-se que

$$
f_{\mathbf{y}}(\mathbf{y} \mid \boldsymbol{\omega})=|\boldsymbol{\Sigma}|^{-1 / 2} g\left(u_{\omega}\right),
$$

com

$$
u_{\omega}=\left(\mathbf{y}-\mathbf{X}_{\omega} \boldsymbol{\beta}\right)^{\top} \boldsymbol{\Sigma}^{-1}\left(\mathbf{y}-\mathbf{X}_{\omega} \boldsymbol{\beta}\right)
$$

e $\mathbf{X}_{\omega}$ sendo a matriz modelo perturbada, com a $i$-ésima coluna dada por $\mathbf{x}_{i \omega}=\mathbf{x}_{i}+s \boldsymbol{\omega}$. Os elementos da matriz $\boldsymbol{\Delta}$ estão descritos no Apêndice D. Os resultados deste esquema de perturbação também podem implementados facilmente.

Com o intuito de ilustrar a metodologia discutida anteriormente, a seguir são apresentados resultados da análise de diagnóstico do exemplo apresentado no Capítulo 3. 


\subsection{Exemplos}

\subsubsection{Continuação do Exemplo 2}

Uma análise de diagnóstico dos resultados obtidos no ajuste do modelo CAPM para os retornos do Itaú é apresentada a seguir, completando-se o processo de identificação de um modelo apropriado aos dados.

O modelo teórico é dado pelas expressões (3.36) e (3.37). Inicialmente, o ajuste supõe que o choque aleatório $\delta_{t} \sim N(0, \phi)$. A Tabela 4.1 e a Figura 4.1 mostram, respectivamente, os parâmetros estimados e um diagrama de dispersão com a reta estimada. Observa-se que, aparentemente, não há discrepâncias relevantes entre a reta ajustada e as observações.

Tabela 4.1: Estimativas dos parâmetros do modelo (3.36) - (3.37) para o Banco Itaú, supondo distribuição normal para os choques aleatórios $\delta$ 's.

\begin{tabular}{crrrr}
\hline Coeficiente & Valor & Erro padrão & Z-valor & P-valor \\
\hline$\beta$ & 0,9883 & 0,0050 & 198,8838 & $<0,0001$ \\
$\rho$ & $-0,2833$ & 0,1078 & & \\
$\phi$ & 0,0045 & 0,0007 & & \\
\hline
\end{tabular}

Inicia-se a análise de diagnóstico com o estudo dos resíduos. A Figura 4.2 mostra os resíduos ordinários padronizados, bem como suas autocorrelações e autocorrelações parciais. Não há "outliers" entre os resíduos, mas as funções de autocorrelação e autocorrelação parcial têm o "lag" 1 estatisticamente diferente de zero. Ou seja, é apropriado considerar um modelo que tenha erros com a estrutura autoregressiva adotada. 


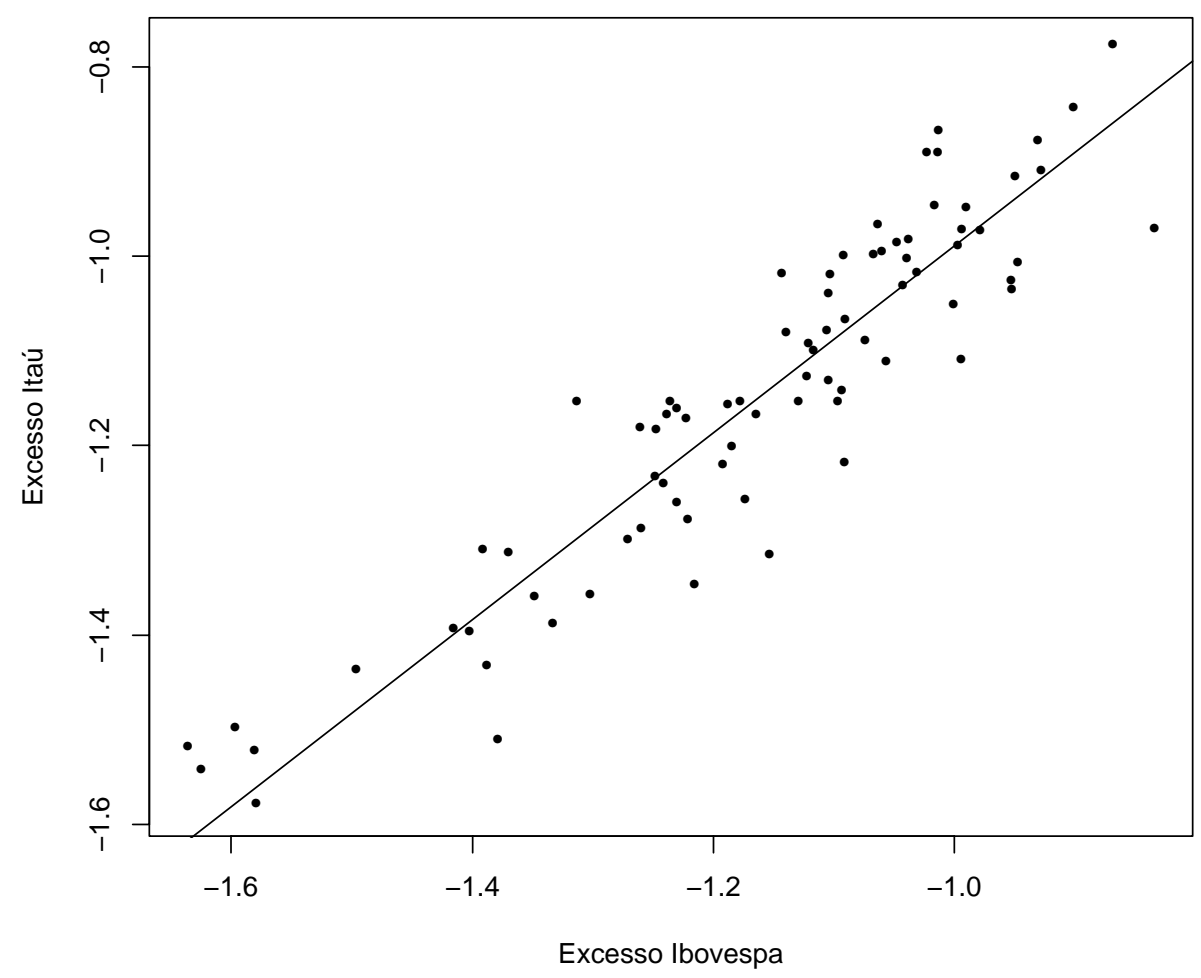

Figura 4.1: Reta ajustada e diagrama de dispersão entre os excessos de retorno do Ibovespa e do Itaú.

Os resíduos definidos para os modelos autoregressivos simétricos dados por (4.5) representam diretamente a distribuição dos choques aleatórios. A Figura 4.3 mostra esses resíduos, bem como suas funções de autocorrelação e autocorrelação parcial. Como esperado, não se tem autocorrelações significativas. No entanto, de acordo com a Figura 4.4, esses resíduos apresentam assimetria a esquerda. 

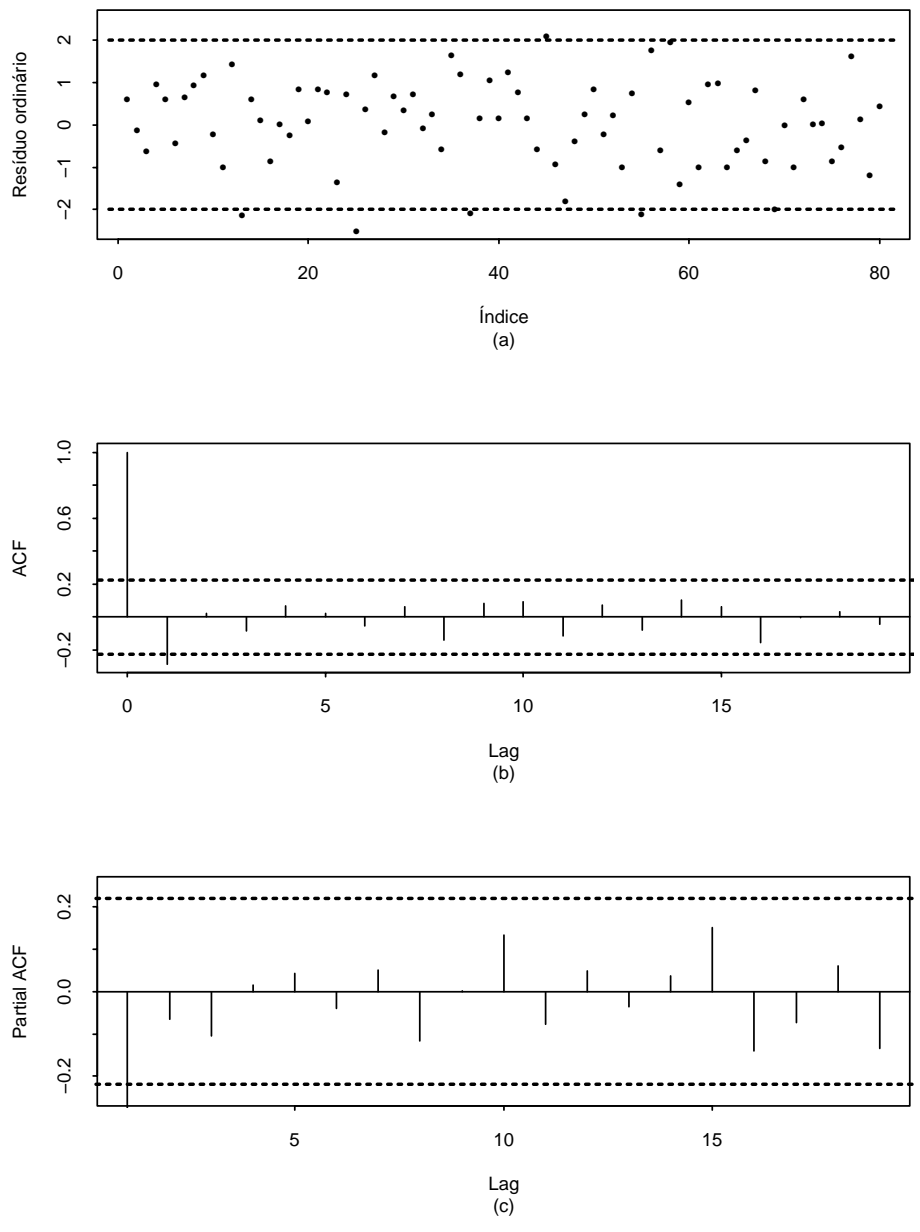

Figura 4.2: (a) Resíduos, (b) função de autocorrelação e (c) função de autocorrelação parcial dos resíduos para o ajuste apresentado pela Tabela 4.1. 


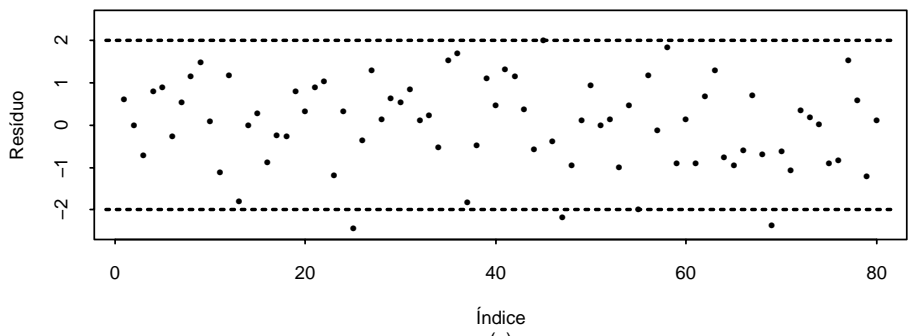

(a)
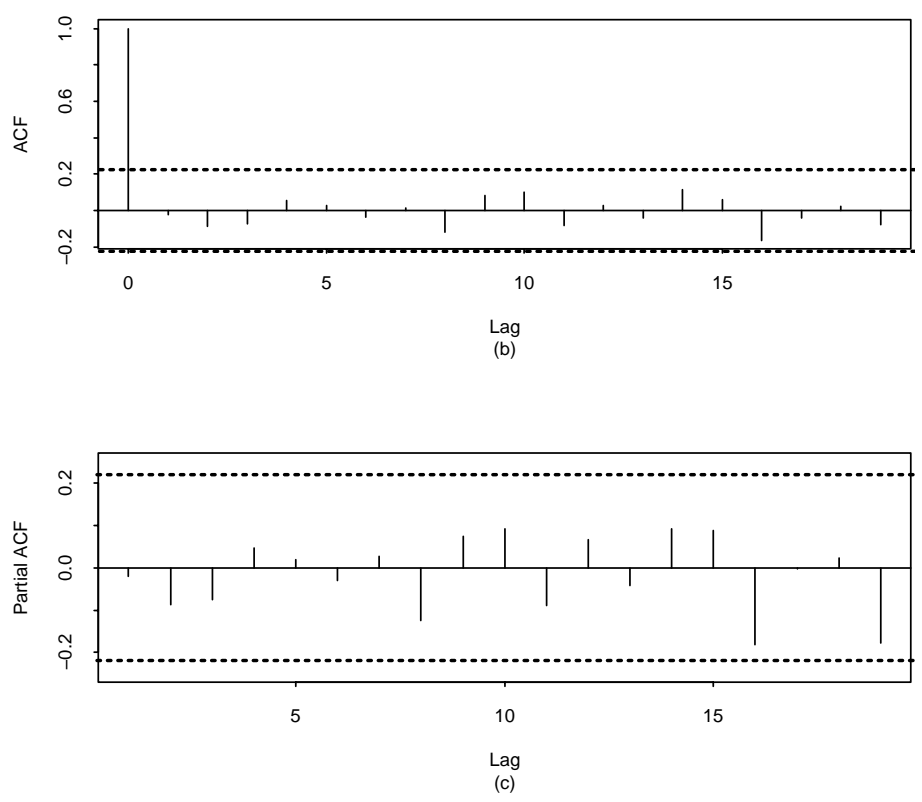

Figura 4.3: (a) Resíduos, (b) função de autocorrelação e (c) função de autocorrelação parcial dos resíduos definidos por (4.5) para o ajuste apresentado pela Tabela 4.1. 

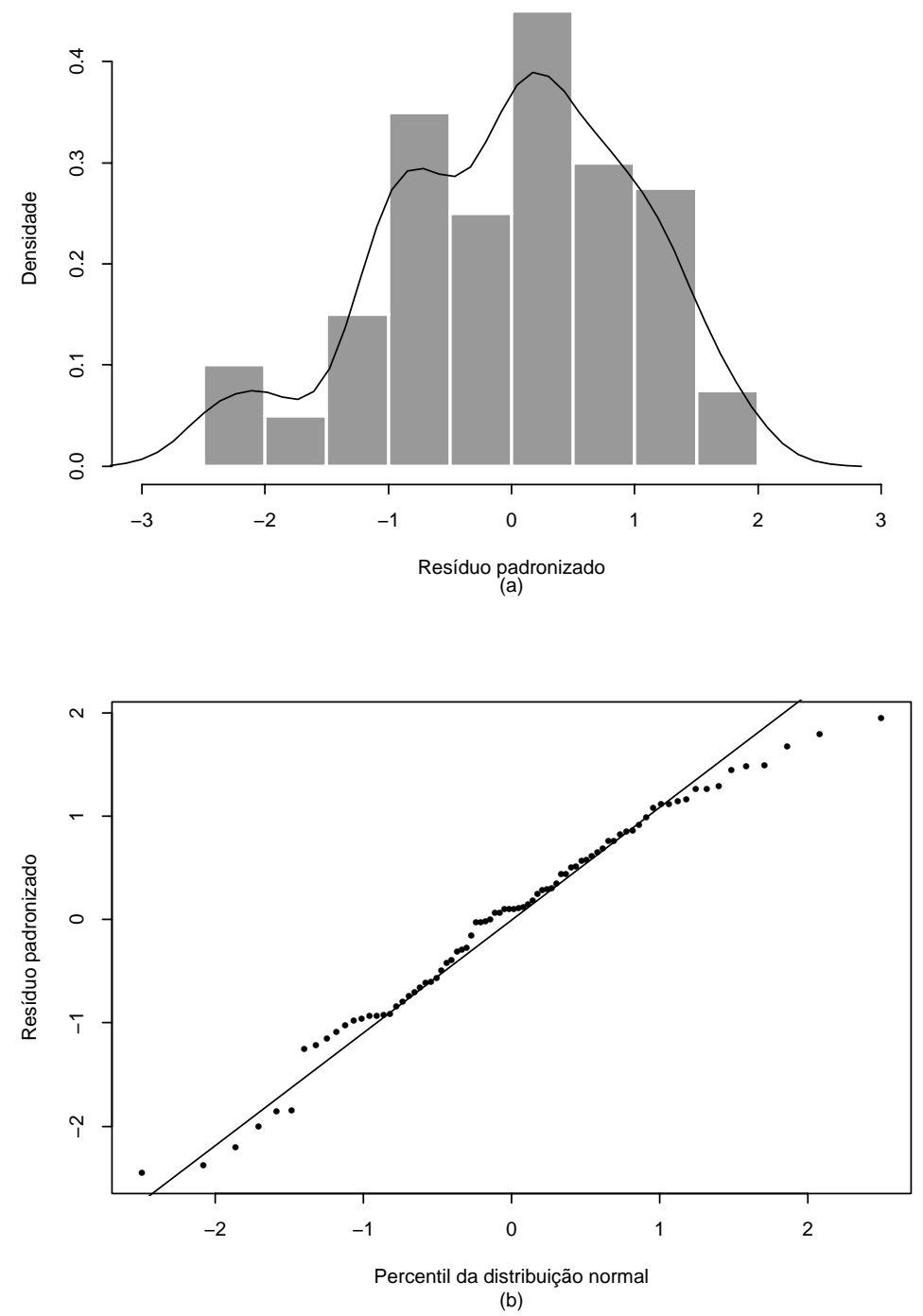

Figura 4.4: (a) Percentis da distribuição normal versus percentis dos resíduos padronizados e (b) histograma dos resíduos padronizados. 
A Figura 4.5 mostra as medidas de alavanca. Destacam-se as observações 8, 9, 10 e 12. Tais observações correspondem ao período em que o mercado brasileiro enfrentou a crise iniciada com a moratória da Rússia em 1998.

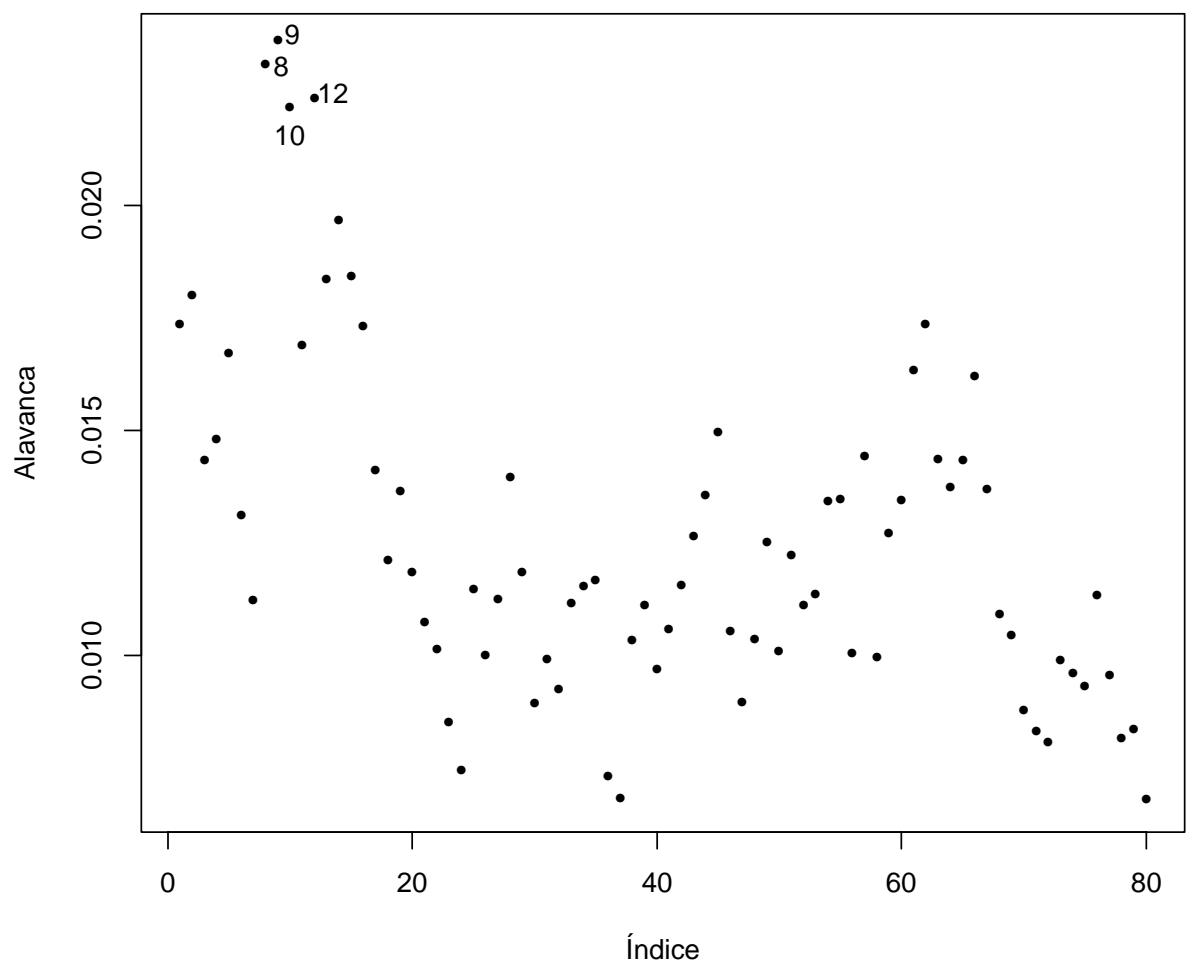

Figura 4.5: Medida de alavanca - Distribuição normal.

Os gráficos com os índices de $\boldsymbol{\ell}_{\max }$ para as perturbações definidas neste capítulo são apresentados pela Figura 4.6. Novamente, destacam-se as observações que correspondem ao período em que o mercado brasileiro encontrava-se na crise provocada pela moratória da Rússia em 1998. Essas observações estão destacadas na Figura 4.7. Observa-se que estes pontos são influentes tanto na estimativa de $\beta$ quanto nas estimativas dos parâmetros $\phi$ e $\rho$. 


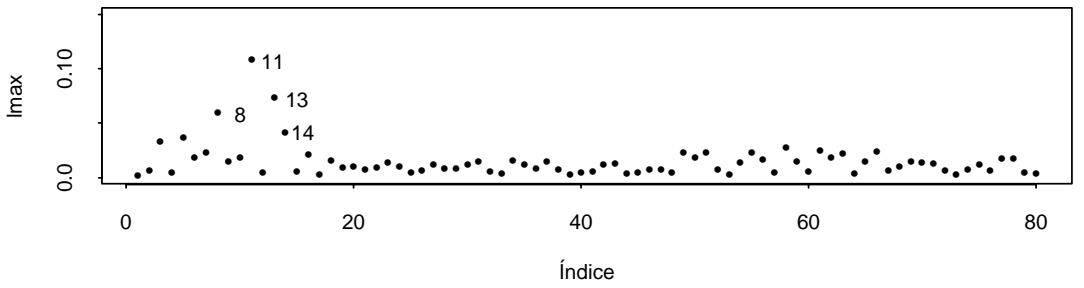

(a)

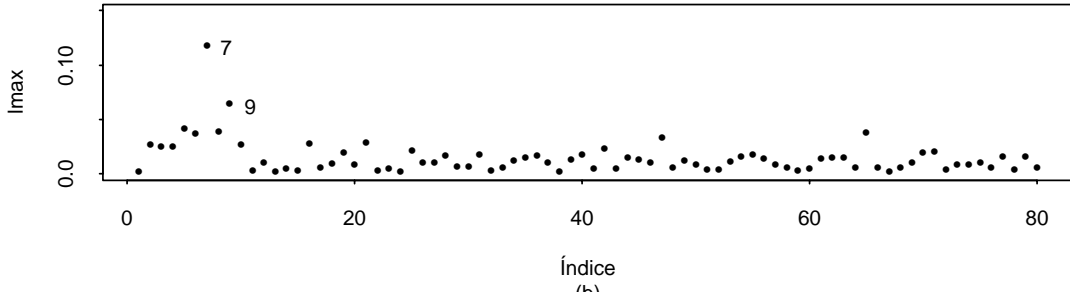

(b)

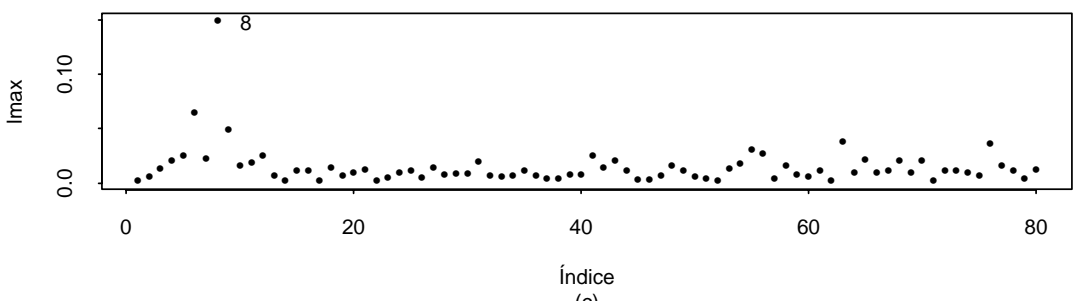

(c)

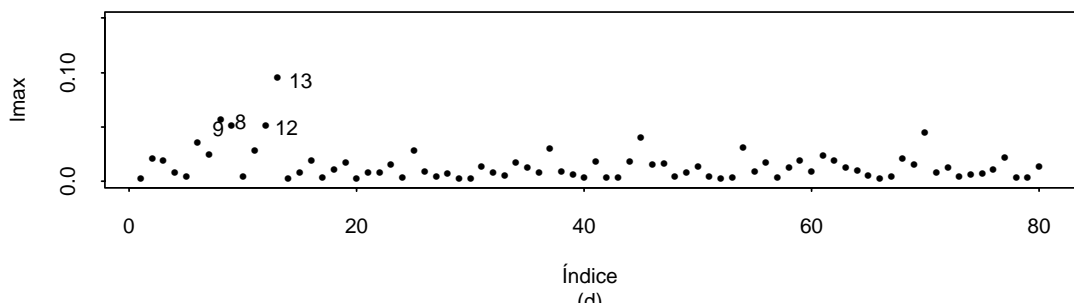

(d)

Figura 4.6: Medidas de influência (distribuição normal): (a) influência da variável resposta (Excesso Itaú) nas estimativas de $\beta$, (b) dos parâmetros $\phi$ e $\rho,(\mathrm{c})$ influência da variável explicativa (Excesso Ibovespa) nas estimativas de $\beta$, (d) dos parâmetros $\phi$ e $\rho$. 


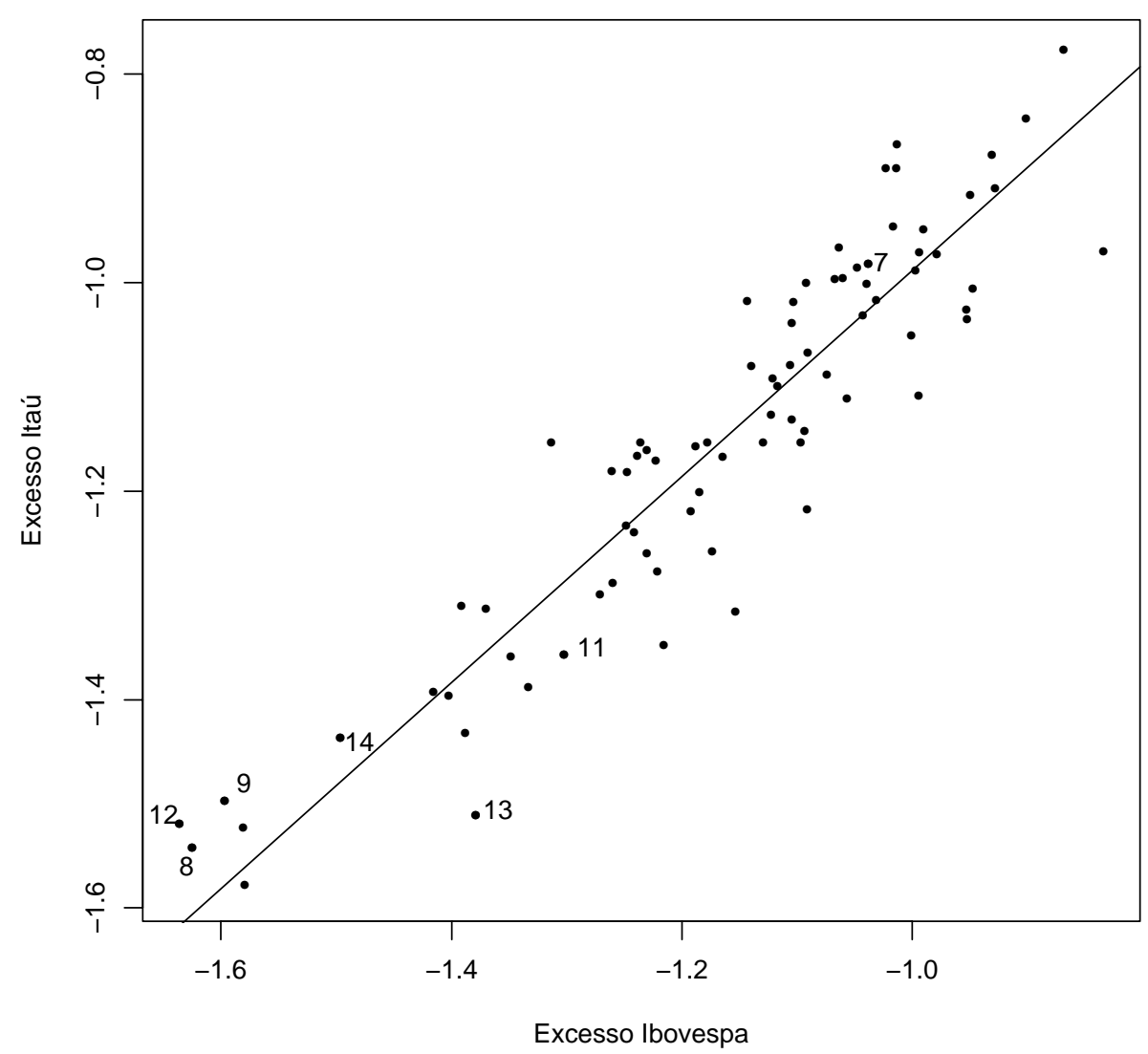

Figura 4.7: Reta ajustada e diagrama de dispersão entre os excessos de retorno do Ibovespa e do Itaú.

Também foram realizados ajustes supondo distribuição $t$-Student para os choques $\delta$ 's. Os resíduos padronizados para os diversos ajustes mantiveram-se, de modo geral, com as mesmas características apresentadas para o modelo normal. Do mesmo modo, os pontos de alavanca também permaneceram os mesmos para os diversos ajustes. Contudo, de acordo com a Figura 4.8 o ajuste de um modelo t-Student com $\nu=5$ foi o que apresentou os melhores resultados com relação as medidas de influência. Comparando-se as Figuras 4.7 e 4.8 nota-se a 
superioridade do modelo $t$ de Student no que diz respeito à robustez da medida de influência contra os esquemas de perturbação adotados. Vale ressaltar que esse ajuste apresenta os mesmos valores (com quatro casas decimais) para as estimativas, esses resultados estão na Tabela 4.2 .

Tabela 4.2: Estimativas dos parâmetros do modelo (3.36) - (3.37) para o Banco Itaú, supondo distribuição $t$-Student para os choques aleatórios $\delta$ 's.

\begin{tabular}{crrrr}
\hline Coeficiente & Valor & Erro padrão & Z-valor & P-valor \\
\hline$\beta$ & 0,9883 & 0,0052 & 190,9916 & $<0,0001$ \\
$\rho$ & $-0,2830$ & 0,1091 & & \\
$\phi$ & 0,0047 & 0,0034 & & \\
\hline
\end{tabular}

Os resultados apresentados na análise de diagnóstico podem ser justificados basicamente a partir da constatação de que as observações que se destacam referem-se ao período da crise iniciada com a moratória da Rússia. 


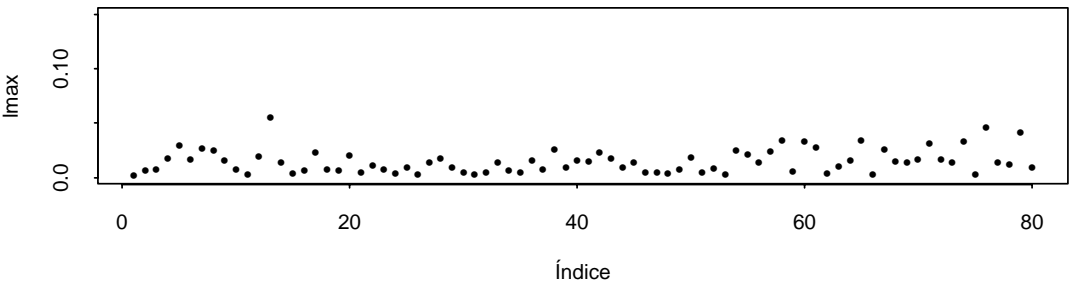

(a)

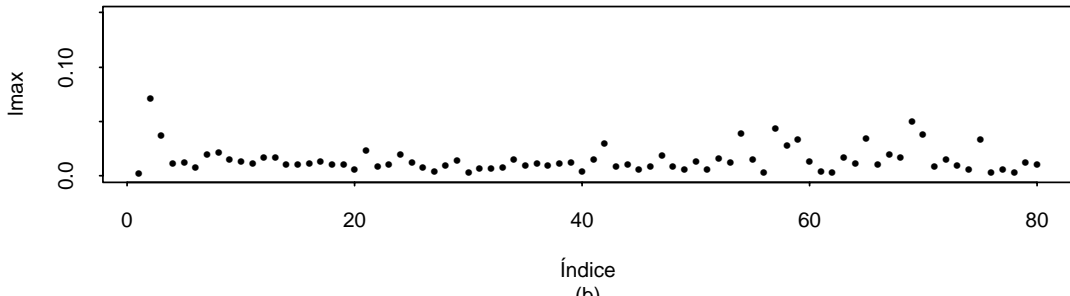

(b)

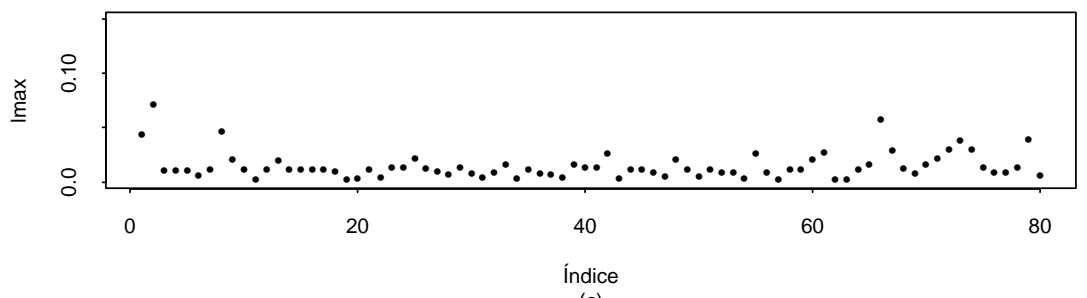

(c)

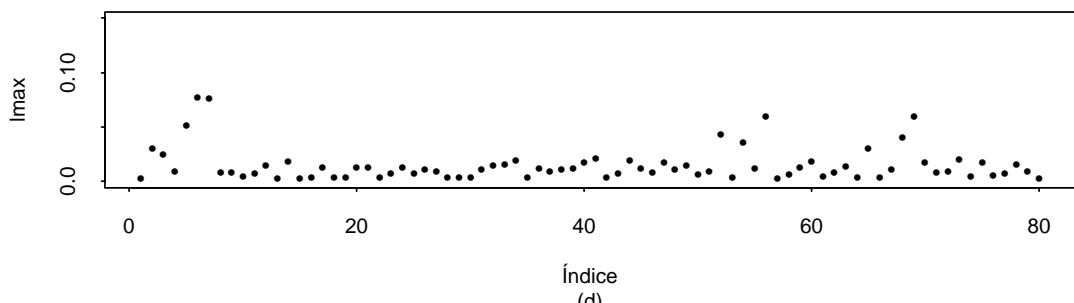

Figura 4.8: Medidas de influência (distribuição t-Student $\operatorname{com} \nu=5$ ): (a) influência da variável resposta (Excesso Itaú) nas estimativas de $\beta$, (b) dos parâmetros $\phi$ e $\rho,(\mathrm{c})$ influência da variável explicativa (Excesso Ibovespa) nas estimativas de $\beta$, (d) dos parâmetros $\phi$ e $\rho$. 
Capítulo 5

\section{Conclusões}

Este trabalho discute técnicas de diagnóstico para os modelos simétricos de regressão linear com erros $\mathrm{AR}(1)$. Os resultados são baseados em resíduos, pontos de alavanca e medidas de influência, de acordo com a literatura. Tendo sido definidos um resíduo e duas perturbações para os modelos. Além disso, foi proposto um algoritmo para estimar os parâmetros do modelo. Os resultados obtidos são de fácil implementação.

Para ilustrar a metodologia, foram usados dados do mercado financeiro brasileiro para uma adaptação do CAPM. A adaptação consiste em tratar o CAPM com erro assumindo uma estrutura AR(1). Os resultados da análise de diagnóstico realizada ratificam a adaptação e os ganhos por substituir a estrutura de erro com uma distribuição normal por uma estrutura equivalente com distribuição $t$-Student são relevantes no que diz respeito a robustez da medida de influência adotada contra os esquemas de perturbação, embora as estimativas permaneçam inalteradas. As observações que se destacaram nas análises de diagnóstico são referentes a um evento de impacto sério nos dados, 
no caso a crise causada pela moratória da Rússia em 1998. Ou seja, as quantidades definidas mostram-se eficientes. O fato dos resíduos e os pontos de alavanca persistirem em ambos os modelos ajustados deve ser inerente ao fenômeno e isto justifica a permanência desses pontos como observações com destaque para essas medidas.

Consideremos como principais contribuições deste trabalho os resultados inferenciais e procedimentos de estimação apresentados no Capítulo 3 e a derivação das curvaturas normais sob alguns esquemas de perturbação, bem como a matriz generalizada de alavanca apresentados no Capítulo 4. Uma extensão natural deste trabalho será a derivação das curvaturas normais e alavanca generalizada em modelos ARMAGARCH com erros elípticos. 
Apêndice A

\section{Vetor gradiente}

Este apêndice contém as expressões que compõem o vetor gradiente do logaritmo da função de verossimilhança para os modelos simétricos de regressão linear com erros $\mathrm{AR}(1)$.

Como foi discutido no Capítulo 3, o logaritmo da função de verossimilhança pode ser expresso como

$$
\mathrm{L}(\boldsymbol{\theta})=-\frac{T}{2} \log (\phi)+\frac{1}{2} \log \left(1-\rho^{2}\right)+\log \{g(u)\},
$$

em que

$$
u=(\mathbf{y}-\mathbf{X} \boldsymbol{\beta})^{\top} \frac{1}{\phi} \mathbf{L}^{\top} \mathbf{L}(\mathbf{y}-\mathbf{X} \boldsymbol{\beta})
$$

A representação (A.1) foi usada para obter-se os resultados apresentados a seguir.

(a) Primeira derivada do logaritmo da função de verossimilhança em relação ao vetor de parâmetros $\beta$

Tem-se que

$$
\frac{\partial \mathrm{L}(\boldsymbol{\theta})}{\partial \boldsymbol{\beta}}=\frac{\partial \log \{g(u)\}}{\partial \boldsymbol{\beta}}=\frac{g^{\prime}(u)}{g(u)} \frac{\partial u}{\partial \boldsymbol{\beta}}
$$


mas

$$
\begin{aligned}
\frac{\partial u}{\partial \boldsymbol{\beta}} & =\frac{\partial}{\partial \boldsymbol{\beta}}\left\{(\mathbf{y}-\mathbf{X} \boldsymbol{\beta})^{\top} \frac{1}{\phi} \mathbf{L}^{\top} \mathbf{L}(\mathbf{y}-\mathbf{X} \boldsymbol{\beta})\right\} \\
& =-\frac{2}{\phi} \mathbf{X}^{\top} \mathbf{L}^{\top} \mathbf{L}(\mathbf{y}-\mathbf{X} \boldsymbol{\beta}) .
\end{aligned}
$$

Portanto,

$$
\begin{aligned}
\frac{\partial \mathrm{L}(\boldsymbol{\theta})}{\partial \boldsymbol{\beta}} & =-\frac{2}{\phi} \frac{g^{\prime}(u)}{g(u)} \mathbf{X}^{\top} \mathbf{L}^{\top} \mathbf{L}(\mathbf{y}-\mathbf{X} \boldsymbol{\beta}) \\
& =-\frac{2}{\phi} W_{g}(u) \mathbf{X}^{\top} \mathbf{L}^{\top} \mathbf{L}(\mathbf{y}-\mathbf{X} \boldsymbol{\beta}) \\
& =\frac{v}{\phi} \mathbf{X}^{\top} \mathbf{L}^{\top} \mathbf{L}(\mathbf{y}-\mathbf{X} \boldsymbol{\beta}) .
\end{aligned}
$$

(b) Primeira derivada do logaritmo da função de verossimilhança em relação ao parâmetro $\phi$

Tem-se que

$$
\begin{aligned}
\frac{\partial \mathrm{L}(\boldsymbol{\theta})}{\partial \phi} & =\frac{\partial}{\partial \phi}\left\{-\frac{T}{2} \log (\phi)+\log \{g(u)\}\right\} \\
& =-\frac{T}{2 \phi}+\frac{g^{\prime}(u)}{g(u)} \frac{\partial u}{\partial \phi}
\end{aligned}
$$

mas

$$
\begin{aligned}
\frac{\partial u}{\partial \phi} & =\frac{\partial}{\partial \phi}\left\{(\mathbf{y}-\mathbf{X} \boldsymbol{\beta})^{\top} \frac{1}{\phi} \mathbf{L}^{\top} \mathbf{L}(\mathbf{y}-\mathbf{X} \boldsymbol{\beta})\right\} \\
& =-\frac{1}{\phi^{2}}(\mathbf{y}-\mathbf{X} \boldsymbol{\beta})^{\top} \mathbf{L}^{\top} \mathbf{L}(\mathbf{y}-\mathbf{X} \boldsymbol{\beta}) .
\end{aligned}
$$

Portanto,

$$
\begin{aligned}
\frac{\partial \mathrm{L}(\boldsymbol{\theta})}{\partial \phi} & =-\frac{T}{2 \phi}-\frac{W_{g}(u)}{\phi^{2}}(\mathbf{y}-\mathbf{X} \boldsymbol{\beta})^{\top} \mathbf{L}^{\top} \mathbf{L}(\mathbf{y}-\mathbf{X} \boldsymbol{\beta}) \\
& =-\frac{T}{2 \phi}-\frac{W_{g}(u)}{\phi} u \\
& =-\frac{1}{\phi}\left\{W_{g}(u) u+\frac{T}{2}\right\}
\end{aligned}
$$


(c) Derivada primeira do logaritmo da função de verossimilhança em relação ao parâmetro $\rho$

Tem-se que

$$
\begin{aligned}
\frac{\partial \mathrm{L}(\boldsymbol{\theta})}{\partial \rho} & =\frac{\partial}{\partial \rho}\left\{\frac{1}{2} \log \left(1-\rho^{2}\right)+\log \{g(u)\}\right\} \\
& =-\frac{\rho}{1-\rho^{2}}+\frac{g^{\prime}(u)}{g(u)} \frac{\partial u}{\partial \rho}
\end{aligned}
$$

mas

$$
\begin{aligned}
\frac{\partial u}{\partial \rho} & =\frac{\partial}{\partial \rho}\left\{(\mathbf{y}-\mathbf{X} \boldsymbol{\beta})^{\top} \frac{1}{\phi} \mathbf{L}^{\top} \mathbf{L}(\mathbf{y}-\mathbf{X} \boldsymbol{\beta})\right\} \\
& =(\mathbf{y}-\mathbf{X} \boldsymbol{\beta})^{\top} \frac{1}{\phi} \frac{\partial \mathbf{L}^{\top} \mathbf{L}}{\partial \rho}(\mathbf{y}-\mathbf{X} \boldsymbol{\beta}) .
\end{aligned}
$$

Portanto,

$$
\begin{aligned}
\frac{\partial \mathrm{L}(\boldsymbol{\theta})}{\partial \rho}= & -\frac{\rho}{1-\rho^{2}}+\frac{g^{\prime}(u)}{g(u)}(\mathbf{y}-\mathbf{X} \boldsymbol{\beta})^{\top} \times \\
& \frac{1}{\phi} \frac{\partial \mathbf{L}^{\top} \mathbf{L}}{\partial \rho}(\mathbf{y}-\mathbf{X} \boldsymbol{\beta}) \\
= & -\frac{\rho}{1-\rho^{2}}+\frac{W_{g}(u)}{\phi} \times \\
& (\mathbf{y}-\mathbf{X} \boldsymbol{\beta})^{\top} \frac{\partial \mathbf{L}^{\top} \mathbf{L}}{\partial \rho}(\mathbf{y}-\mathbf{X} \boldsymbol{\beta}) .
\end{aligned}
$$


Apêndice B

\section{Matriz hessiana}

Este apêndice contém as expressões que compõem a matriz hessiana do logaritmo da função de verossimilhança para os modelos simétricos de regressão linear com erros $\mathrm{AR}(1)$.

Como discutido no Capítulo 3, o logaritmo da função de verossimilhança pode ser expresso como

$$
\mathrm{L}(\boldsymbol{\theta})=-\frac{T}{2} \log (\phi)+\frac{1}{2} \log \left(1-\rho^{2}\right)+\log \{g(u)\},
$$

em que

$$
u=(\mathbf{y}-\mathbf{X} \boldsymbol{\beta})^{\top} \frac{1}{\phi} \mathbf{L}^{\top} \mathbf{L}(\mathbf{y}-\mathbf{X} \boldsymbol{\beta})
$$

A representação (B.1) foi usada para obter-se os resultados apresentados a seguir. Além disso, $\mathbf{e}=(\mathbf{y}-\mathbf{X} \beta)$. 
(a) Derivadas cruzadas do logaritmo da função de verossimilhança em relação ao vetor $\beta$

Tem-se que

$$
\begin{aligned}
\frac{\partial^{2} \mathbf{L}(\boldsymbol{\theta})}{\partial \boldsymbol{\beta} \partial \boldsymbol{\beta}^{\top}}= & \frac{\partial}{\partial \boldsymbol{\beta}^{\top}}\left\{-\frac{2}{\phi} W_{g}(u) \mathbf{X}^{\top} \mathbf{L}^{\top} \mathbf{L}(\mathbf{y}-\mathbf{X} \boldsymbol{\beta})\right\} \\
= & -\frac{2}{\phi} \mathbf{X}^{\top} \mathbf{L}^{\top} \mathbf{L}(\mathbf{y}-\mathbf{X} \boldsymbol{\beta}) W_{g}^{\prime}(u) \frac{\partial u}{\partial \boldsymbol{\beta}^{\top}}+ \\
& \frac{2}{\phi} W_{g}(u) \mathbf{X}^{\top} \mathbf{L}^{\top} \mathbf{L} \mathbf{X},
\end{aligned}
$$

mas

$$
\frac{\partial u}{\partial \boldsymbol{\beta}^{\top}}=-\frac{2}{\phi}(\mathbf{y}-\mathbf{X} \boldsymbol{\beta})^{\top} \mathbf{L}^{\top} \mathbf{L} \mathbf{X} .
$$

Portanto,

$$
\begin{aligned}
\frac{\partial^{2} \mathbf{L}(\boldsymbol{\theta})}{\partial \boldsymbol{\beta} \partial \boldsymbol{\beta}^{\top}}= & \frac{2}{\phi} \mathbf{X}^{\top} \mathbf{L}^{\top} \mathbf{L} \mathbf{e} W_{g}^{\prime}(u) \frac{2}{\phi} \mathbf{e}^{\top} \mathbf{L}^{\top} \mathbf{L} \mathbf{X}+ \\
& \frac{2}{\phi} W_{g}(u) \mathbf{X}^{\top} \mathbf{L}^{\top} \mathbf{L} \mathbf{X} \\
= & \frac{2}{\phi} \mathbf{X}^{\top} \mathbf{L}^{\top} \mathbf{L} \times \\
& \left\{W_{g}(u)+\frac{2}{\phi} W_{g}^{\prime}(u) \mathbf{e} \mathbf{e}^{\top} \mathbf{L}^{\top} \mathbf{L}\right\} \mathbf{X} .
\end{aligned}
$$

(b) Derivadas cruzadas do logaritmo da função de verossimilhança em relação ao vetor de parâmetros $\beta$ e ao parâmetro $\phi$

Tem-se que

$$
\begin{aligned}
\frac{\partial^{2} \mathrm{~L}(\boldsymbol{\theta})}{\partial \boldsymbol{\beta} \partial \phi^{\top}}= & \frac{\partial}{\partial \phi^{\top}}\left\{-\frac{2}{\phi} W_{g}(u) \mathbf{X}^{\top} \mathbf{L}^{\top} \mathbf{L}(\mathbf{y}-\mathbf{X} \boldsymbol{\beta})\right\} \\
= & -\frac{2}{\phi} \mathbf{X}^{\top} \mathbf{L}^{\top} \mathbf{L}(\mathbf{y}-\mathbf{X} \boldsymbol{\beta}) W_{g}^{\prime}(u) \frac{\partial u}{\partial \phi^{\top}}+ \\
& \frac{2}{\phi^{2}} W_{g}(u) \mathbf{X}^{\top} \mathbf{L}^{\top} \mathbf{L}(\mathbf{y}-\mathbf{X} \boldsymbol{\beta})
\end{aligned}
$$


mas

$$
\frac{\partial u}{\partial \phi^{\top}}=-\frac{1}{\phi^{2}}(\mathbf{y}-\mathbf{X} \boldsymbol{\beta})^{\top} \mathbf{L}^{\top} \mathbf{L}(\mathbf{y}-\mathbf{X} \boldsymbol{\beta})
$$

Portanto,

$$
\begin{aligned}
\frac{\partial^{2} \mathbf{L}(\boldsymbol{\theta})}{\partial \boldsymbol{\beta} \partial \phi^{\top}=} & \frac{2}{\phi} \mathbf{X}^{\top} \mathbf{L}^{\top} \mathbf{L}(\mathbf{y}-\mathbf{X} \boldsymbol{\beta}) W_{g}^{\prime}(u) \times \\
& \frac{1}{\phi^{2}}(\mathbf{y}-\mathbf{X} \boldsymbol{\beta})^{\top} \mathbf{L}^{\top} \mathbf{L}(\mathbf{y}-\mathbf{X} \boldsymbol{\beta})+ \\
& \frac{2}{\phi^{2}} W_{g}(u) \mathbf{X}^{\top} \mathbf{L}^{\top} \mathbf{L}(\mathbf{y}-\mathbf{X} \boldsymbol{\beta}) \\
= & \frac{2}{\phi^{2}} \mathbf{X}^{\top} \mathbf{L}^{\top} \mathbf{L}(\mathbf{y}-\mathbf{X} \boldsymbol{\beta}) \times \\
& \left\{W_{g}(u)+W_{g}^{\prime}(u) u\right\}
\end{aligned}
$$

(c) Derivadas cruzadas do logaritmo da função de verossimilhança em relação ao vetor de parâmetros $\beta$ e ao parâmetro $\rho$

Tem-se que

$$
\begin{aligned}
\frac{\partial^{2} \mathbf{L}(\boldsymbol{\theta})}{\partial \boldsymbol{\beta} \partial \rho^{\top}}= & \frac{\partial}{\partial \rho^{\top}}\left\{-\frac{2}{\phi} W_{g}(u) \mathbf{X}^{\top} \mathbf{L}^{\top} \mathbf{L}(\mathbf{y}-\mathbf{X} \boldsymbol{\beta})\right\} \\
= & -\frac{2}{\phi} \mathbf{X}^{\top} \mathbf{L}^{\top} \mathbf{L}(\mathbf{y}-\mathbf{X} \boldsymbol{\beta}) W_{g}^{\prime}(u) \frac{\partial u}{\partial \rho^{\top}} \\
& -\frac{2}{\phi^{2}} W_{g}(u) \mathbf{X}^{\top} \frac{\partial \mathbf{L}^{\top} \mathbf{L}}{\partial \rho^{\top}}(\mathbf{y}-\mathbf{X} \boldsymbol{\beta}),
\end{aligned}
$$

mas

$$
\frac{\partial u}{\partial \rho^{\top}}=(\mathbf{y}-\mathbf{X} \boldsymbol{\beta})^{\top} \frac{1}{\phi} \frac{\partial \mathbf{L}^{\top} \mathbf{L}}{\partial \rho^{\top}}(\mathbf{y}-\mathbf{X} \boldsymbol{\beta}) .
$$


Portanto,

$$
\begin{aligned}
\frac{\partial^{2} \mathbf{L}(\boldsymbol{\theta})}{\partial \boldsymbol{\beta} \partial \rho^{\top}}= & -\frac{2}{\phi} \mathbf{X}^{\top} \mathbf{L}^{\top} \mathbf{L}(\mathbf{y}-\mathbf{X} \boldsymbol{\beta}) W_{g}^{\prime}(u)(\mathbf{y}-\mathbf{X} \boldsymbol{\beta})^{\top} \times \\
& \frac{1}{\phi} \frac{\partial \mathbf{L}^{\top} \mathbf{L}}{\partial \rho^{\top}}(\mathbf{y}-\mathbf{X} \boldsymbol{\beta})-\frac{2}{\phi} W_{g}(u) \mathbf{X}^{\top} \times \\
& \frac{\partial \mathbf{L}^{\top} \mathbf{L}}{\partial \rho^{\top}}(\mathbf{y}-\mathbf{X} \boldsymbol{\beta}) \\
= & -\frac{2}{\phi} \mathbf{X}^{\top}\left\{W_{g}(u)+\frac{W_{g}^{\prime}(u)}{\phi} \mathbf{L}^{\top} \mathbf{L} \mathbf{e} \mathbf{e}^{\top}\right\} \times \\
& \frac{\partial \mathbf{L}^{\top} \mathbf{L}}{\partial \rho^{\top}} \mathbf{e} .
\end{aligned}
$$

(d) Segunda derivada do logaritmo da função de verossimilhança em relação ao parâmetro $\phi$

Tem-se que

$$
\begin{aligned}
\frac{\partial^{2} \mathbf{L}(\boldsymbol{\theta})}{\partial \phi \partial \phi^{\top}}= & \frac{\partial}{\partial \phi^{\top}}\left\{-\frac{T}{2 \phi}-\frac{W_{g}(u)}{\phi^{2}} \mathbf{e}^{\top} \mathbf{L}^{\top} \mathbf{L} \mathbf{e}\right\} \\
= & \frac{T}{2 \phi^{2}}-\frac{1}{\phi^{2}} \mathbf{e}^{\top} \mathbf{L}^{\top} \mathbf{L} \mathbf{e} W_{g}^{\prime}(u) \frac{\partial u}{\partial \phi^{\top}}+ \\
& \frac{2}{\phi^{3}} W_{g}(u) \mathbf{e}^{\top} \mathbf{L}^{\top} \mathbf{L} \mathbf{e},
\end{aligned}
$$

mas

$$
\frac{\partial u}{\partial \phi^{\top}}=-\frac{1}{\phi^{2}}(\mathbf{y}-\mathbf{X} \boldsymbol{\beta})^{\top} \mathbf{L}^{\top} \mathbf{L}(\mathbf{y}-\mathbf{X} \boldsymbol{\beta})
$$

Portanto,

$$
\begin{aligned}
\frac{\partial^{2} \mathbf{L}(\boldsymbol{\theta})}{\partial \phi \partial \phi^{\top}}= & \frac{T}{2 \phi^{2}}+\frac{2}{\phi^{3}} W_{g}(u) \mathbf{e}^{\top} \mathbf{L}^{\top} \mathbf{L} \mathbf{e} \\
& \frac{1}{\phi^{2}} \mathbf{e}^{\top} \mathbf{L}^{\top} \mathbf{L} \mathbf{e} W_{g}^{\prime}(u) \frac{1}{\phi^{2}} \mathbf{e}^{\top} \mathbf{L}^{\top} \mathbf{L} \mathbf{e} \\
= & \frac{1}{\phi^{2}}\left\{u\left\{2 W_{g}(u)+u W_{g}^{\prime}(u)\right\}+\frac{T}{2}\right\} .
\end{aligned}
$$


(e) Derivada cruzada do logaritmo da função de verossimilhança em relação aos parâmetros $\phi$ e $\rho$

Tem-se que

$$
\begin{aligned}
\frac{\partial^{2} \mathbf{L}(\boldsymbol{\theta})}{\partial \phi \partial \rho^{\top}}= & \frac{\partial}{\partial \rho^{\top}}\left\{-\frac{T}{2 \phi}-\frac{W_{g}(u)}{\phi^{2}} \mathbf{e}^{\top} \mathbf{L}^{\top} \mathbf{L} \mathbf{e}\right\} \\
= & -\frac{1}{\phi^{2}} W_{g}(u) \mathbf{e}^{\top} \frac{\partial \mathbf{L}^{\top} \mathbf{L}}{\partial \rho^{\top}} \mathbf{e}-\frac{1}{\phi^{2}} \mathbf{e}^{\top} \mathbf{L}^{\top} \mathbf{L} \mathbf{e} \times \\
& W_{g}^{\prime}(u) \frac{\partial u}{\partial \rho^{\top}}
\end{aligned}
$$

mas

$$
\frac{\partial u}{\partial \rho^{\top}}=(\mathbf{y}-\mathbf{X} \boldsymbol{\beta})^{\top} \frac{1}{\phi} \frac{\partial \mathbf{L}^{\top} \mathbf{L}}{\partial \rho^{\top}}(\mathbf{y}-\mathbf{X} \boldsymbol{\beta}) .
$$

Portanto,

$$
\begin{aligned}
\frac{\partial^{2} \mathbf{L}(\boldsymbol{\theta})}{\partial \phi \partial \rho^{\top}}= & -\frac{1}{\phi^{2}} W_{g}(u) \mathbf{e}^{\top} \frac{\partial \mathbf{L}^{\top} \mathbf{L}}{\partial \rho^{\top}} \mathbf{e} \\
& -\frac{1}{\phi^{2}} \mathbf{e}^{\top} \mathbf{L}^{\top} \mathbf{L} \mathbf{e} W_{g}^{\prime}(u) \mathbf{e}^{\top} \frac{1}{\phi} \frac{\partial \mathbf{L}^{\top} \mathbf{L}}{\partial \rho^{\top}} \mathbf{e} \\
= & -\frac{1}{\phi^{2}}\left\{W_{g}(u)+u W_{g}^{\prime}(u)\right\} \mathbf{e}^{\top} \frac{\partial \mathbf{L}^{\top} \mathbf{L}}{\partial \rho} \mathbf{e} .
\end{aligned}
$$

(f) Segunda derivada do logaritmo da função de verossimilhança em relação ao parâmetro $\rho$

Tem-se que

$$
\begin{aligned}
\frac{\partial^{2} \mathbf{L}(\boldsymbol{\theta})}{\partial \rho \partial \rho^{\top}}= & \frac{\partial}{\partial \rho^{\top}}\left\{-\frac{\rho}{1-\rho^{2}}+\frac{W_{g}(u)}{\phi} \mathbf{e}^{\top} \frac{\partial \mathbf{L}^{\top} \mathbf{L}}{\partial \rho} \mathbf{e}\right\} \\
= & -\frac{1+\rho^{2}}{\left(1-\rho^{2}\right)^{2}}+\frac{W_{g}(u)}{\phi} \mathbf{e}^{\top} \frac{\partial^{2} \mathbf{L}^{\top} \mathbf{L}}{\partial \rho \partial \rho^{\top}} \mathbf{e} \\
& \frac{1}{\phi} \mathbf{e}^{\top} \frac{\partial \mathbf{L}^{\top} \mathbf{L}}{\partial \rho} \mathbf{e} W_{g}^{\prime}(u) \frac{\partial u}{\partial \rho^{\top}},
\end{aligned}
$$


mas

$$
\frac{\partial u}{\partial \rho^{\top}}=(\mathbf{y}-\mathbf{X} \boldsymbol{\beta})^{\top} \frac{1}{\phi} \frac{\partial \mathbf{L}^{\top} \mathbf{L}}{\partial \rho^{\top}}(\mathbf{y}-\mathbf{X} \boldsymbol{\beta}) .
$$

Portanto,

$$
\begin{aligned}
\frac{\partial^{2} \mathbf{L}(\boldsymbol{\theta})}{\partial \rho \partial \rho^{\top}}= & -\frac{1+\rho^{2}}{\left(1-\rho^{2}\right)^{2}}+\frac{W_{g}(u)}{\phi} \mathbf{e}^{\top} \frac{\partial^{2} \mathbf{L}^{\top} \mathbf{L}}{\partial \rho \partial \rho^{\top}} \mathbf{e} \\
& \frac{1}{\phi} \mathbf{e}^{\top} \frac{\partial \mathbf{L}^{\top} \mathbf{L}}{\partial \rho} \mathbf{e} W_{g}^{\prime}(u) \mathbf{e}^{\top} \frac{1}{\phi} \frac{\partial \mathbf{L}^{\top} \mathbf{L}}{\partial \rho^{\top}} \mathbf{e} \\
= & -\frac{1+\rho^{2}}{\left(1-\rho^{2}\right)^{2}}+\frac{W_{g}(u)}{\phi} \mathbf{e}^{\top} \frac{\partial^{2} \mathbf{L}^{\top} \mathbf{L}}{\partial \rho \partial \rho^{\top}} \mathbf{e} \\
& \frac{W_{g}^{\prime}(u)}{\phi^{2}}\left\{\mathbf{e}^{\top} \frac{\partial \mathbf{L}^{\top} \mathbf{L}}{\partial \rho} \mathbf{e}\right\}^{2} .
\end{aligned}
$$


Apêndice C

\section{Pontos de alavanca}

Para se determinar pontos de alavanca é necessário calcular as matrizes $\mathbf{D}_{\theta}$ e $\ddot{\mathbf{L}}_{\theta \mathbf{y}}$. Este apêndice apresenta as expressões que compõem essas matrizes para os modelos simétricos de regressão linear com erros $\mathrm{AR}(1)$. O vetor $\mathbf{e}=(\mathbf{y}-\mathbf{X} \beta)$ e a representação da verossimilhança (B.1) são usados para obter os resultados que se seguem.

\section{(a) Matriz $\mathbf{D}_{\theta}$}

Tem-se que

$$
\mathbf{D}_{\theta}=\partial \boldsymbol{\mu} / \partial \boldsymbol{\theta}^{\top}
$$

Portanto,

$$
\begin{aligned}
\partial \boldsymbol{\mu} / \partial \boldsymbol{\beta}^{\top} & =\partial(\mathbf{X} \boldsymbol{\beta}) / \partial \boldsymbol{\beta}^{\top} \\
\partial \boldsymbol{\mu} / \partial \phi^{\top} & =\partial(\mathbf{X} \boldsymbol{\beta}) / \partial \phi^{\top} \\
& =\mathbf{0} \mathrm{e} \\
\partial \boldsymbol{\mu} / \partial \rho^{\top} & =\partial(\mathbf{X} \boldsymbol{\beta}) / \partial \rho^{\top} \\
& =\mathbf{0} .
\end{aligned}
$$

Dos resultados (C.2) - (C.3), $\mathbf{D}_{\theta}$ é dada por

$$
\mathbf{D}_{\theta}=(\mathbf{X}, \mathbf{0}, \mathbf{0}) \text {. }
$$


(b) Matriz $\ddot{\mathbf{L}}_{\theta \mathbf{y}}$

Tem-se que

$$
\ddot{\mathbf{L}}_{\theta \mathbf{y}}=\left[\begin{array}{c}
\ddot{\mathbf{L}}_{\beta \mathbf{y}} \\
\ddot{\mathbf{L}}_{\phi \mathbf{y}} \\
\ddot{\mathbf{L}}_{\rho \mathbf{y}}
\end{array}\right],
$$

em que

$$
\begin{aligned}
\ddot{\mathbf{L}}_{\beta \mathbf{y}} & =\frac{\partial^{2} \mathrm{~L}(\boldsymbol{\theta})}{\partial \boldsymbol{\beta} \partial \mathbf{y}^{\top}}, \\
\ddot{\mathbf{L}}_{\phi \mathbf{y}} & =\frac{\partial^{2} \mathrm{~L}(\boldsymbol{\theta})}{\partial \phi \partial \mathbf{y}^{\top}}
\end{aligned}
$$

e

$$
\ddot{\mathbf{L}}_{\rho \mathbf{y}}=\frac{\partial^{2} \mathrm{~L}(\boldsymbol{\theta})}{\partial \rho \partial \mathbf{y}^{\top}} .
$$

- $\ddot{\mathbf{L}}_{\beta \mathbf{y}}$ : do Apêndice A, tem-se que

$$
\frac{\partial \mathrm{L}(\boldsymbol{\theta})}{\partial \boldsymbol{\beta}}=-\frac{2}{\phi} W_{g}(u) \mathbf{X}^{\top} \mathbf{L}^{\top} \mathbf{L}(\mathbf{y}-\mathbf{X} \boldsymbol{\beta}) .
$$

Portanto,

$$
\begin{aligned}
\frac{\partial^{2} \mathrm{~L}(\boldsymbol{\theta})}{\partial \boldsymbol{\beta} \partial \mathbf{y}^{\top}}= & \frac{\partial}{\partial \mathbf{y}^{\top}}\left\{-\frac{2}{\phi} W_{g}(u) \mathbf{X}^{\top} \mathbf{L}^{\top} \mathbf{L}(\mathbf{y}-\mathbf{X} \boldsymbol{\beta})\right\} \\
= & -\frac{2}{\phi} \mathbf{X}^{\top} \mathbf{L}^{\top} \mathbf{L}(\mathbf{y}-\mathbf{X} \boldsymbol{\beta}) W_{g}^{\prime}(u) \frac{\partial u}{\partial \mathbf{y}^{\top}} \\
& -\frac{2}{\phi} W_{g}(u) \mathbf{X}^{\top} \mathbf{L}^{\top} \mathbf{L},
\end{aligned}
$$

mas

$$
\frac{\partial u}{\partial \mathbf{y}^{\top}}=\frac{2}{\phi}(\mathbf{y}-\mathbf{X} \boldsymbol{\beta})^{\top} \mathbf{L}^{\top} \mathbf{L} .
$$


Então,

$$
\begin{aligned}
\frac{\partial^{2} \mathbf{L}(\boldsymbol{\theta})}{\partial \boldsymbol{\beta} \partial \mathbf{y}^{\top}}= & -\frac{2}{\phi} \mathbf{X}^{\top} \mathbf{L}^{\top} \mathbf{L}(\mathbf{y}-\mathbf{X} \boldsymbol{\beta}) W_{g}^{\prime}(u) \frac{2}{\phi}(\mathbf{y}-\mathbf{X} \boldsymbol{\beta})^{\top} \mathbf{L}^{\top} \mathbf{L} \\
& -\frac{2}{\phi} W_{g}(u) \mathbf{X}^{\top} \mathbf{L}^{\top} \mathbf{L} \\
= & -\frac{2}{\phi} \mathbf{X}^{\top} \mathbf{L}^{\top} \mathbf{L}\left\{W_{g}(u)+\frac{2}{\phi} W_{g}^{\prime}(u) \mathbf{e} \mathbf{e}^{\top} \mathbf{L}^{\top} \mathbf{L}\right\}(\mathrm{C} .12)
\end{aligned}
$$

- $\ddot{\mathbf{L}}_{\phi \mathbf{y}}$ : Do Apêndice A, tem-se que

$$
\frac{\partial \mathrm{L}(\boldsymbol{\theta})}{\partial \phi}=-\frac{T}{2 \phi}-\frac{W_{g}(u)}{\phi} u .
$$

Portanto,

$$
\begin{aligned}
\frac{\partial^{2} \mathrm{~L}(\boldsymbol{\theta})}{\partial \phi \partial \mathbf{y}^{\top}} & =\frac{\partial}{\partial \mathbf{y}^{\top}}\left\{-\frac{T}{2 \phi}-\frac{W_{g}(u)}{\phi} u\right\} \\
& =-\frac{W_{g}(u)}{\phi} \frac{\partial u}{\partial \mathbf{y}^{\top}}-\frac{W_{g}^{\prime}(u)}{\phi} u \frac{\partial u}{\partial \mathbf{y}^{\top}} \\
& =-\frac{1}{\phi}\left\{W_{g}(u)+W_{g}^{\prime}(u) u\right\} \frac{\partial u}{\partial \mathbf{y}^{\top}} \\
& =-\frac{2}{\phi^{2}}\left\{W_{g}(u)+W_{g}^{\prime}(u) u\right\} \mathbf{e}^{\top} \mathbf{L}^{\top} \mathbf{L} .
\end{aligned}
$$

- $\ddot{\mathbf{L}}_{\rho \mathbf{y}}$ : Do Apêndice A, tem-se que

$$
\frac{\partial \mathrm{L}(\boldsymbol{\theta})}{\partial \rho}=-\frac{\rho}{1-\rho^{2}}+\frac{W_{g}(u)}{\phi} \mathbf{e}^{\top} \frac{\partial \mathbf{L}^{\top} \mathbf{L}}{\partial \rho} \mathbf{e} .
$$

Portanto,

$$
\begin{aligned}
\frac{\partial^{2} \mathbf{L}(\boldsymbol{\theta})}{\partial \rho \partial \mathbf{y}^{\top}}= & \frac{\partial}{\partial \mathbf{y}^{\top}}\left\{-\frac{\rho}{1-\rho^{2}}+\frac{W_{g}(u)}{\phi} \mathbf{e}^{\top} \frac{\partial \mathbf{L}^{\top} \mathbf{L}}{\partial \rho} \mathbf{e}\right\} \\
= & \frac{1}{\phi} \mathbf{e}^{\top} \frac{\partial \mathbf{L}^{\top} \mathbf{L}}{\partial \rho} \mathbf{e} W_{g}^{\prime}(u) \frac{\partial u}{\partial \mathbf{y}^{\top}}+ \\
& \frac{W_{g}(u)}{\phi} \frac{\partial}{\partial \mathbf{y}^{\top}}\left\{\mathbf{e}^{\top} \frac{\partial \mathbf{L}^{\top} \mathbf{L}}{\partial \rho} \mathbf{e}\right\},
\end{aligned}
$$


mas

$$
\frac{\partial}{\partial \mathbf{y}^{\top}}\left(\mathbf{e}^{\top} \frac{\partial \mathbf{L}^{\top} \mathbf{L}}{\partial \rho} \mathbf{e}\right)=2(\mathbf{y}-\mathbf{X} \boldsymbol{\beta})^{\top} \frac{\partial \mathbf{L}^{\top} \mathbf{L}}{\partial \rho^{\top}} .
$$

Então,

$$
\begin{aligned}
\frac{\partial^{2} \mathbf{L}(\boldsymbol{\theta})}{\partial \rho \partial \mathbf{y}^{\top}}= & \frac{1}{\phi} \mathbf{e}^{\top} \frac{\partial \mathbf{L}^{\top} \mathbf{L}}{\partial \rho} \mathbf{e} W_{g}^{\prime}(u) \frac{2}{\phi}(\mathbf{y}-\mathbf{X} \boldsymbol{\beta})^{\top} \mathbf{L}^{\top} \mathbf{L}+ \\
& \frac{W_{g}(u)}{\phi} 2(\mathbf{y}-\mathbf{X} \boldsymbol{\beta})^{\top} \frac{\partial \mathbf{L}^{\top} \mathbf{L}}{\partial \rho} \\
= & \frac{2}{\phi} \mathbf{e}^{\top}\left\{W_{g}(u) \frac{\partial \mathbf{L}^{\top} \mathbf{L}}{\partial \rho}+\frac{W_{g}^{\prime}(u)}{\phi} \mathbf{e}^{\top} \frac{\partial \mathbf{L}^{\top} \mathbf{L}}{\partial \rho} \mathbf{e} \mathbf{L}^{\top} \mathbf{L}\right\} \cdot(\text { C. } 23)
\end{aligned}
$$


Apêndice D

\section{Influência local}

Para avaliar a curvatura normal é necessário calcular a matriz $\Delta$ para os esquemas de perturbação. Este apêndice apresenta as expressões que compõem essa matriz para as perturbações definidas no Capítulo 4.

(a) Perturbação aditiva na resposta do modelo simétrico de regressão com erro $\operatorname{AR}(1)$

Para a perturbação aditiva no vetor de respostas $\mathbf{y}_{\omega}=\mathbf{y}+s \boldsymbol{\omega}$, em que $s$ é uma medida de escala, a densidade do modelo fica dada por

$$
f_{\mathbf{y}}(\mathbf{y} \mid \boldsymbol{\omega})=|\boldsymbol{\Sigma}|^{-1 / 2} g\left(u_{\omega}\right)
$$

em que

$$
u_{\omega}=(\mathbf{y}+s \boldsymbol{\omega}-\mathbf{X} \boldsymbol{\beta})^{\top} \boldsymbol{\Sigma}^{-1}(\mathbf{y}+s \boldsymbol{\omega}-\mathbf{X} \boldsymbol{\beta}) .
$$

A partir de (D.1) obtém-se uma expressão análoga a (B.1), que é a representação da verossimilhança usada para obter os resultados que se sequem. O vetor $\mathbf{e}_{\omega}=(\mathbf{y}+s \boldsymbol{\omega}-\mathbf{X} \boldsymbol{\beta})$ também é usado nos resultados. 
- Derivadas cruzadas do logaritmo da função de verossimilhança perturbada em relação ao vetor de parâmetros $\beta$ e ao vetor de perturbação $\omega$

De acordo com os resultados do Apêndice A, tem-se que

$$
\frac{\partial \mathrm{L}(\boldsymbol{\theta} \mid \boldsymbol{\omega})}{\partial \boldsymbol{\beta}}=-\frac{2}{\phi} W_{g}\left(u_{\omega}\right) \mathbf{X}^{\top} \mathbf{L}^{\top} \mathbf{L} \mathbf{e}_{\omega}
$$

Conseqüentemente,

$$
\begin{aligned}
\frac{\partial^{2} \mathbf{L}(\boldsymbol{\theta} \mid \boldsymbol{\omega})}{\partial \boldsymbol{\beta} \partial \boldsymbol{\omega}^{\top}}= & \frac{\partial}{\partial \boldsymbol{\omega}^{\top}}\left\{-\frac{2}{\phi} W_{g}\left(u_{\omega}\right) \mathbf{X}^{\top} \mathbf{L}^{\top} \mathbf{L} \mathbf{e}_{\omega}\right\} \\
= & -\frac{2}{\phi} \mathbf{X}^{\top} \mathbf{L}^{\top} \mathbf{L} \mathbf{e}_{\omega} W_{g}^{\prime}\left(u_{\omega}\right) \frac{\partial u_{\omega}}{\partial \boldsymbol{\omega}^{\top}} \\
& -\frac{2 s}{\phi} W_{g}\left(u_{\omega}\right) \mathbf{X}^{\top} \mathbf{L}^{\top} \mathbf{L},
\end{aligned}
$$

mas

$$
\frac{\partial u_{\omega}}{\partial \mathbf{y}^{\top}}=\frac{2 s}{\phi} \mathbf{e}_{\omega}^{\top} \mathbf{L}^{\top} \mathbf{L}
$$

Portanto,

$$
\begin{aligned}
\frac{\partial^{2} \mathbf{L}(\boldsymbol{\theta} \mid \boldsymbol{\omega})}{\partial \boldsymbol{\beta} \partial \boldsymbol{\omega}^{\top}}= & -\frac{2}{\phi} \mathbf{X}^{\top} \mathbf{L}^{\top} \mathbf{L} \mathbf{e}_{\omega} W_{g}^{\prime}(\omega) \frac{2 s}{\phi} \mathbf{e}_{\omega}^{\top} \mathbf{L}^{\top} \mathbf{L} \\
& -\frac{2 s}{\phi} W_{g}\left(u_{\omega}\right) \mathbf{X}^{\top} \mathbf{L}^{\top} \mathbf{L} \\
& -\frac{2 s}{\phi} \mathbf{X}^{\top} \mathbf{L}^{\top} \mathbf{L}\left\{W_{g}\left(u_{\omega}\right)+\frac{2}{\phi} W_{g}^{\prime}\left(u_{\omega}\right) \mathbf{e}_{\omega} \mathbf{e}_{\omega}^{\top} \mathbf{L}^{\top} \mathbf{L}\right\}
\end{aligned}
$$

- Derivadas cruzadas do logaritmo da função de verossimilhança perturbada em relação ao parâmetro $\phi$ e ao vetor de perturbação $\omega$

Segue-se, do Apêndice A, que

$$
\frac{\partial \mathrm{L}(\boldsymbol{\theta} \mid \boldsymbol{\omega})}{\partial \phi}=-\frac{T}{2 \phi}-\frac{W_{g}\left(u_{\omega}\right)}{\phi} u .
$$


Conseqüentemente,

$$
\begin{aligned}
\frac{\partial^{2} \mathrm{~L}(\boldsymbol{\theta} \mid \boldsymbol{\omega})}{\partial \boldsymbol{\beta} \partial \boldsymbol{\omega}^{\top}} & =\frac{\partial}{\partial \boldsymbol{\omega}^{\top}}\left\{-\frac{T}{2 \phi}-\frac{W_{g}\left(u_{\omega}\right)}{\phi} u_{\omega}\right\} \\
& =-\frac{1}{\phi}\left\{W_{g}\left(u_{\omega}\right)+W_{g}^{\prime}\left(u_{\omega}\right) u_{\omega}\right\} \frac{\partial u_{\omega}}{\partial \boldsymbol{\omega}^{\top}} \\
& =-\frac{1}{\phi}\left\{W_{g}\left(u_{\omega}\right)+W_{g}^{\prime}\left(u_{\omega}\right) u_{\omega}\right\} \frac{2 s}{\phi} \mathbf{e}_{\omega}^{\top} \mathbf{L}^{\top} \mathbf{L}( \\
& =-\frac{2 s}{\phi^{2}}\left\{W_{g}\left(u_{\omega}\right)+W_{g}^{\prime}\left(u_{\omega}\right) u_{\omega}\right\} \mathbf{e}_{\omega}^{\top} \mathbf{L}^{\top} \mathbf{L}
\end{aligned}
$$

- Derivadas cruzadas do logaritmo da função de verossimilhança perturbada em relação ao parâmetro $\rho$ e ao vetor de perturbação $\omega$

Segue-se, do Apêndice A, que

$$
\frac{\partial \mathrm{L}(\boldsymbol{\theta} \mid \boldsymbol{\omega})}{\partial \rho}=-\frac{\rho}{1-\rho^{2}}+\frac{W_{g}\left(u_{\omega}\right)}{\phi} \mathbf{e}_{\omega}^{\top} \frac{\partial \mathbf{L}^{\top} \mathbf{L}}{\partial \rho} \mathbf{e}_{\omega} .
$$

Conseqüentemente,

$$
\begin{aligned}
\frac{\partial^{2} \mathbf{L}(\boldsymbol{\theta} \mid \boldsymbol{\omega})}{\partial \phi \partial \boldsymbol{\omega}^{\top}}= & \frac{\partial}{\partial \boldsymbol{\omega}^{\top}}\left\{\frac{W_{g}\left(u_{\omega}\right)}{\phi} \mathbf{e}_{\omega}^{\top} \frac{\partial \mathbf{L}^{\top} \mathbf{L}}{\partial \rho} \mathbf{e}_{\omega}\right\} \\
= & \frac{1}{\phi} \mathbf{e}_{\omega}^{\top} \frac{\partial \mathbf{L}^{\top} \mathbf{L}}{\partial \rho} \mathbf{e}_{\omega} W_{g}^{\prime}\left(u_{\omega}\right) \frac{\partial u_{\omega}}{\partial \boldsymbol{\omega}^{\top}}+ \\
& \frac{W_{g}\left(u_{\omega}\right)}{\phi} \frac{\partial}{\partial \boldsymbol{\omega}^{\top}}\left\{\mathbf{e}_{\omega}^{\top} \frac{\partial \mathbf{L}^{\top} \mathbf{L}}{\partial \rho} \mathbf{e}_{\omega}\right\}
\end{aligned}
$$

Mas,

$$
\frac{\partial}{\partial \boldsymbol{\omega}^{\top}}\left\{\mathbf{e}_{\omega}^{\top} \frac{\partial \mathbf{L}^{\top} \mathbf{L}}{\partial \rho} \mathbf{e}_{\omega}\right\}=2 s(\mathbf{y}+s \boldsymbol{\omega}-\mathbf{X} \boldsymbol{\beta})^{\top} \frac{\partial \mathbf{L}^{\top} \mathbf{L}}{\partial \rho} .
$$


Portanto,

$$
\begin{aligned}
\frac{\partial^{2} \mathbf{L}(\boldsymbol{\theta} \mid \boldsymbol{\omega})}{\partial \rho \partial \boldsymbol{\omega}^{\top}}= & \frac{1}{\phi} \mathbf{e}_{\omega}^{\top} \frac{\partial \mathbf{L}^{\top} \mathbf{L}}{\partial \rho} \mathbf{e}_{\omega} W_{g}^{\prime}\left(u_{\omega}\right) \frac{2 s}{\phi}(\mathbf{y}+s \boldsymbol{\omega}-\mathbf{X} \boldsymbol{\beta})^{\top} \mathbf{L}^{\top} \mathbf{L}+ \\
& \frac{W_{g}\left(u_{\omega}\right)}{\phi} 2 s(\mathbf{y}+s \boldsymbol{\omega}-\mathbf{X} \boldsymbol{\beta})^{\top} \frac{\partial \mathbf{L}^{\top} \mathbf{L}}{\partial \rho} \\
= & \frac{2 s}{\phi} \mathbf{e}_{\omega}^{\top} \frac{\partial \mathbf{L}^{\top} \mathbf{L}}{\partial \rho}\left\{W_{g}\left(u_{\omega}\right)+\frac{W_{g}^{\prime}\left(u_{\omega}\right)}{\phi} \mathbf{e}_{\omega} \mathbf{e}_{\omega}^{\top} \mathbf{L}^{\top} \mathbf{L}\right\}
\end{aligned}
$$

(b) Perturbação aditiva em uma variável explicativa contínua do modelo simétrico de regressão com erro $\operatorname{AR}(1)$

Para a perturbação aditiva da variável explicativa contínua $\mathbf{x}_{i}=$ $\mathbf{x}_{i}+s \boldsymbol{\omega}$, em que $s$ é uma medida de escala, a densidade do modelo fica dada por

$$
f_{\mathbf{y}}(\mathbf{y} \mid \boldsymbol{\omega})=|\boldsymbol{\Sigma}|^{-1 / 2} g\left(u_{\omega}\right)
$$

com

$$
u_{\omega}=\left(\mathbf{y}-\mathbf{X}_{\omega} \boldsymbol{\beta}\right)^{\top} \boldsymbol{\Sigma}^{-1}\left(\mathbf{y}-\mathbf{X}_{\omega} \boldsymbol{\beta}\right)
$$

e $\mathbf{X}_{\omega}$ é a matriz modelo perturbada, ou seja, $\mathbf{X}_{\omega}$ tem a $i$-ésima coluna dada por $\mathbf{x}_{i \omega}=\mathbf{x}_{i}+s \boldsymbol{\omega}$.

- Derivadas cruzadas do logaritmo da função de verossimilhança perturbada em relação ao vetor de parâmetros $\beta$ e ao vetor de perturbação $\omega$

De acordo com os resultados do Apêndice A, tem-se que

$$
\frac{\partial \mathrm{L}(\boldsymbol{\theta} \mid \boldsymbol{\omega})}{\partial \boldsymbol{\beta}}=-\frac{2}{\phi} W_{g}\left(u_{\omega}\right) \mathbf{X}_{\omega}^{\top} \mathbf{L}^{\top} \mathbf{L}\left(\mathbf{y}-\mathbf{X}_{\omega} \boldsymbol{\beta}\right)
$$


Segue-se que

$$
\begin{aligned}
\frac{\partial^{2} \mathbf{L}(\boldsymbol{\theta} \mid \boldsymbol{\omega})}{\partial \boldsymbol{\beta} \partial \omega_{j}}= & \frac{\partial}{\partial \omega_{j}}\left\{-\frac{2}{\phi} W_{g}\left(u_{\omega}\right) \mathbf{X}_{\omega}^{\top} \mathbf{L}^{\top} \mathbf{L}\left(\mathbf{y}-\mathbf{X}_{\omega} \boldsymbol{\beta}\right)\right\} \\
= & -\frac{2}{\phi} \mathbf{X}_{\omega}^{\top} \mathbf{L}^{\top} \mathbf{L}\left(\mathbf{y}-\mathbf{X}_{\omega} \boldsymbol{\beta}\right) W_{g}^{\prime}\left(u_{\omega}\right) \frac{\partial u_{\omega}}{\partial \boldsymbol{\omega}^{\top}} \\
& -\frac{2}{\phi} W_{g}\left(u_{\omega}\right) \frac{\partial \mathbf{X}_{\omega}^{\top}}{\partial \omega_{j}} \mathbf{L}^{\top} \mathbf{L}\left(\mathbf{y}-\mathbf{X}_{\omega} \boldsymbol{\beta}\right) \\
& -\frac{2}{\phi} W_{g}\left(u_{\omega}\right) \mathbf{X}_{\omega}^{\top} \mathbf{L}^{\top} \mathbf{L} \frac{\partial\left(\mathbf{y}-\mathbf{X}_{\omega} \boldsymbol{\beta}\right)}{\partial \omega_{j}}
\end{aligned}
$$

mas

$$
\frac{\partial u_{\omega}}{\partial \omega_{j}}=-\frac{2}{\phi}\left(\mathbf{y}-\mathbf{X}_{\omega} \boldsymbol{\beta}\right)^{\top} \mathbf{L}^{\top} \mathbf{L} \frac{\partial \mathbf{X}_{\omega}^{\top}}{\partial \omega_{j}} \boldsymbol{\beta}
$$

Portanto,

$$
\begin{aligned}
\frac{\partial^{2} \mathrm{~L}(\boldsymbol{\theta} \mid \boldsymbol{\omega})}{\partial \boldsymbol{\beta} \partial \omega_{j}}= & \frac{4}{\phi^{2}} \mathbf{X}_{\omega}^{\top} \mathbf{L}^{\top} \mathbf{L}\left(\mathbf{y}-\mathbf{X}_{\omega} \boldsymbol{\beta}\right) W_{g}^{\prime}\left(u_{\omega}\right)\left(\mathbf{y}-\mathbf{X}_{\omega} \boldsymbol{\beta}\right)^{\top} \mathbf{L}^{\top} \mathbf{L} \frac{\partial \mathbf{X}_{\omega}^{\top}}{\partial \omega_{j}} \boldsymbol{\beta} \\
& -\frac{2}{\phi} W_{g}\left(u_{\omega}\right) \frac{\partial \mathbf{X}_{\omega}^{\top}}{\partial \omega_{j}} \mathbf{L}^{\top} \mathbf{L}\left(\mathbf{y}-\mathbf{X}_{\omega} \boldsymbol{\beta}\right) \\
& +\frac{2}{\phi} W_{g}\left(u_{\omega}\right) \mathbf{X}_{\omega}^{\top} \mathbf{L}^{\top} \mathbf{L} \frac{\partial \mathbf{X}_{\omega}}{\partial \omega_{j}} \boldsymbol{\beta} .
\end{aligned}
$$

- Derivadas cruzadas do logaritmo da função de verossimilhança perturbada em relação ao parâmetro $\phi$ e ao vetor de perturbação $\omega$

Segue-se, do Apêndice A, que

$$
\frac{\partial \mathrm{L}(\boldsymbol{\theta} \mid \boldsymbol{\omega})}{\partial \phi}=-\frac{T}{2 \phi}-\frac{W_{g}\left(u_{\omega}\right)}{\phi} u_{\omega}
$$

Conseqüentemente,

$$
\begin{aligned}
\frac{\partial^{2} \mathrm{~L}(\boldsymbol{\theta} \mid \boldsymbol{\omega})}{\partial \phi \partial \omega_{j}} & =\frac{\partial}{\partial \boldsymbol{\omega}^{\top}}\left\{-\frac{T}{2 \phi}-\frac{W_{g}\left(u_{\omega}\right)}{\phi} u\right\} \\
& =-\frac{1}{\phi}\left(W_{g}\left(u_{\omega}\right)+W_{g}^{\prime}\left(u_{\omega}\right) u_{\omega}\right) \frac{\partial u_{\omega}}{\partial \boldsymbol{\omega}^{\top}} \\
& =\frac{2}{\phi^{2}}\left(W_{g}\left(u_{\omega}\right)+W_{g}^{\prime}\left(u_{\omega}\right) u_{\omega}\right) \mathbf{e}_{\omega}^{\top} \mathbf{L}^{\top} \mathbf{L} \frac{\partial \mathbf{X}_{\omega}^{\top}}{\partial \omega_{j}} \boldsymbol{\beta} .
\end{aligned}
$$


- Derivadas cruzadas do logaritmo da função de verossimilhança perturbada em relação ao parâmetro $\rho$ e ao vetor de perturbação $\omega$

Segue-se, do Apêndice A, que

$$
\frac{\partial \mathbf{L}(\boldsymbol{\theta} \mid \boldsymbol{\omega})}{\partial \rho}=-\frac{\rho}{1-\rho^{2}}+\frac{W_{g}\left(u_{\omega}\right)}{\phi} \mathbf{e}^{\top} \frac{\partial \mathbf{L}^{\top} \mathbf{L}}{\partial \rho} \mathbf{e} .
$$

Portanto,

$$
\begin{aligned}
\frac{\partial^{2} \mathbf{L}(\boldsymbol{\theta} \mid \boldsymbol{\omega})}{\partial \phi \partial \omega_{j}}= & \frac{\partial}{\partial \omega_{j}}\left\{\frac{W_{g}\left(u_{\omega}\right)}{\phi} \mathbf{e}^{\top} \frac{\partial \mathbf{L}^{\top} \mathbf{L}}{\partial \rho} \mathbf{e}\right\} \\
= & \frac{1}{\phi} \mathbf{e}^{\top} \frac{\partial \mathbf{L}^{\top} \mathbf{L}}{\partial \rho} \mathbf{e} W_{g}^{\prime}\left(u_{\omega}\right) \frac{\partial u_{\omega}}{\partial \omega_{j}}+ \\
& \frac{W_{g}\left(u_{\omega}\right)}{\phi} \frac{\partial}{\partial \omega_{j}}\left\{\mathbf{e}^{\top} \frac{\partial \mathbf{L}^{\top} \mathbf{L}}{\partial \rho} \mathbf{e}\right\} .
\end{aligned}
$$

Mas

$$
\frac{\partial}{\partial \boldsymbol{\omega}^{\top}}\left\{\mathbf{e}^{\top} \frac{\partial \mathbf{L}^{\top} \mathbf{L}}{\partial \rho} \mathbf{e}\right\}=-2\left(\mathbf{y}-\mathbf{X}_{\omega} \boldsymbol{\beta}\right)^{\top} \frac{\partial \mathbf{L}^{\top} \mathbf{L}}{\partial \rho} \frac{\partial \mathbf{X}_{\omega}^{\top}}{\partial \omega_{j}} \boldsymbol{\beta}
$$

Portanto,

$$
\begin{aligned}
\frac{\partial^{2} \mathrm{~L}(\boldsymbol{\theta} \mid \boldsymbol{\omega})}{\partial \rho \partial \boldsymbol{\omega}^{\top}}= & \frac{1}{\phi} \mathbf{e}^{\top} \frac{\partial \mathbf{L}^{\top} \mathbf{L}}{\partial \rho} \mathbf{e} W_{g}^{\prime}\left(u_{\omega}\right) \frac{(-2)}{\phi}\left(\mathbf{y}-\mathbf{X}_{\omega} \boldsymbol{\beta}\right)^{\top} \mathbf{L}^{\top} \mathbf{L} \frac{\partial \mathbf{X}_{\omega}^{\top}}{\partial \omega_{j}} \boldsymbol{\beta} \\
& \frac{W_{g}\left(u_{\omega}\right)}{\phi}(-2)\left(\mathbf{y}-\mathbf{X}_{\omega} \boldsymbol{\beta}\right)^{\top} \frac{\partial \mathbf{L}^{\top} \mathbf{L}}{\partial \rho} \frac{\partial \mathbf{X}_{\omega}^{\top}}{\partial \omega_{j}} \boldsymbol{\beta} \\
= & -\frac{2}{\phi} \mathbf{e}^{\top} \frac{\partial \mathbf{L}^{\top} \mathbf{L}}{\partial \rho}\left\{W_{g}\left(u_{\omega}\right)+\frac{W_{g}^{\prime}\left(u_{\omega}\right)}{\phi} \mathbf{e}^{\top} \mathbf{L}^{\top} \mathbf{L}\right\} \mathrm{x} \\
& \frac{\partial \mathbf{X}_{\omega}^{\top}}{\partial \omega_{j}} \boldsymbol{\beta} .
\end{aligned}
$$




\section{Referências Bibliográficas}

Anderson, T. W. e Fang, K. T (1987) Cochran's Theorem for Elliptically Contourned Distributions. Sankhya A, Vol. 49, 305-315.

Arellano-Valle, R. B. (1994) Distribuições Elípticas: Propriedades e Aplicações no Modelo de Regressão. Tese de doutorado, Departamento de Estatística, Universidade de São Paulo, Brasil.

Atkinson, A. C. (1985) Plots, Transformation and Regression. Clarendon Press: Oxford.

Berkane, M. e Bentler, P. M. (1986) Moments of Elliptical Distributed Random Variates. Statistics and Probability Letters, Vol. 4, 333-335.

Cademartori, D.; Romo, C.; Campos, R e Galea, M. (2003) Robust Estimation of Systematic Risk Using the $t$ Distribution in the Chilean Stock Markets. Applied Economics Letters, Vol. 10, 447-453.

Cambanis, S.; Huang, S. e Simons, G. (1981) On the Theory of Elliptically Contoured Distributions. Journal of Multivariate Analysis, Vol. 11, 368-385.

Chatterjee, S. e Hadi, A. S. (1988) Sensitivity Analysis in Linear Regression. New York: Wiley.

Cook, R. D. (1986) Assessment of Local Influence (with discussion). Journal of the Royal Statistical Society B, Vol. 48, 133-169.

Cook, R. D. e Weisberg, S. (1982) Residuals and Influence in Regression. Chapman and Hall. 
Cook, R. D. e Weisberg, S. (1983) Diagnostics for Heteroscedasticity in Regression. Biometrika, Vol. 70, 1-10.

Cox, D. R. e Snell, E. J. (1968) A General Definition of Residuals (with discussion). Journal of the Royal Statistical Society B, Vol. 30, 248-275.

Cysneiros, F. J. A. (2004) Métodos Restritos e Validação de Modelos Simétricos de Regressão. Tese de doutorado, Departamento de Estatística, Universidade de São Paulo, Brasil.

Cysneiros, F. J. A.; Paula, G. A. e Galea, M. (2005) Modelos Simétricos Aplicados, Texto de Minicurso da 9a. Escola de Modelos de Regressão.

Elton, E. e Gruber M. (1995). Modern Portfolio Theory and Investment Analysis. Wiley.

Emerson, J. D.; Hoaglin, D. C. e Kempthorne, P. J. (1984) Leverage in Least Squares Additive-Plus-Multiplicative Fits for Two-Way Tables. Journal of the American Statistical Association, Vol. 79, 329-335.

Engels, M. (2004) Portfolio Optimization: Beyond Markowitz. Master's Theses, Leiden University.

Fang, K.; Kotz, S. e Ng, K. (1990) Symmetric Multivariate and Related Distributions. Chapman and Hall.

Galea, M.; Paula, G. A. e Bolfarine, H. (1997) Local Infuence in Elliptical Linear Regression Models. The Statistician, Vol. 46, 71-79.

Galea M.; Paula G. A. e Uribe-Opazo M. (2003) On influence diagnostic in univariate elliptical linear regression models, Statistical Papers, Vol. 44, 23-45

Harvey, A. C. e Phillips, G. D. A. (1979) Maximum Likelihood Estimation of Regression Models with Autoregresssive-Moving Average Disturbances. Biometrika, Vol. 66, 49-58.

Harville, D. A. (1997) Matrix Algebra From a Statistician's Perspective. Springer-Verlag, New York.

Haslett, J. e Kevin, H. (1998) Residuals for the Linear Model General Covariance Structure. Journal of the Royal Statistical Society, Vol. 60, 201-215. 
Hoaglin, D. C. e Welsch, R. E. (1978) The Hat Matrix in Regression and ANOVA. The American Statistician, Vol. 32, 17-22.

Kim, S. e Huggins R. (1998) Diagnostics for Autocorrelated Regression Models. Austral. \& New Zealand J. Statist. Vol. 40, 65-71.

Landsman, V. (2002) Tail Conditional Expectations for Elliptical Distributions. Disponível em: http://stat.haifa.ac.il/ landsman/ articles.

Lesaffre, E. e Verbeke, G. (1998) Local Influence in Linear Mixed Models. Biometrics, Vol. 54, 570-582.

Lintner, J. (1965) The Valuation of Risk Assets and the Selection of Risky Investments in Stock Portfolios and Capital Budgets. Review of Economics and Statistics, Vol. 41, 13-37.

Liu, S. (2000) On Local Influence for Elliptical Linear Models. Statistical Papers, Vol. 41, 211-224.

Liu, S. (2002) Local Influence in Multivariate Elliptical Linear Regression Models. Linear Algebra and Its Aplications, Vol. 354, 159-174.

Liu, S. (2004) On Diagnostics in Conditionally Heteroskedastic Time Series Models Under Elliptical Distributions. Journal of Applied Probability, Vol. 41A, 393-403.

Magnus, J. R. e Neudecker, H. (1997) Matrix Differential Calculus with Applications in Statistics and Econometrics. Wiley.

McCullagh, P. e Nelder, J. A. (1989) Generalized Linear Models, 2nd Edition. Chapman and Hall.

Montgomery, D. C.; Peck, E. A. e Vining, G. G. (2001) Introduction to Linear Regression Analysis. Third Edition, John Wiley.

Mossin, J. (1966) Equilibruium in a capital asset market. Econometrica, Vol. 35, 768-783.

Mendes, B. V. M (2004) Introdução à Análise de Eventos Extremos. E-papers. 
Muirhead, R. (1980) The Efects of Symmetric Distributions on Some Standard Procedures Involving Correlation Coefficients. Multivariate Statistical Analysis (ed. R. P. Gupta) North-Holland, 143-159.

Muirhead, R. (1982) Aspects of Multivariate Statistical Theory. NewYork: John Wiley.

Neter, J.; Kutner, M. H.; Nachtsheim, C. J. e Wasserman, W. (1996) Applied Linear Regression Models, 3rd Edition. Irwin.

Ortega, E. M. M.; Bolfarine, H. e Paula G. A. (2003) Influence Diagnostic in Generalized Log-gamma Regression Models. Computational Statistics and Data Analysis, Vol. 42, 165-186.

Paula. G. A (20040 Modelos de regressão com apoio computacional. Instituto de Matemática e Estatística da Universidade de São Paulo.

Paula G. A.; Cysneiros F. J. A. e Galea M.; (2003) Local Influence and Leverage in Elliptical Nonlinear Regression Models. In: Proceedings of the 18th International Workshop on Statistical Modelling, Verbeke, G., Molenberghs, G., Aerts, A. and Fieuws, S. (Eds.). Leuven: Katholieke Universiteit Leuven, 361-365.

Poon, W. Y. e Poon, Y. S. (1999) Conformal Normal Curvature and Assessment of Local Infuence. Journal of Royal Statistical Society, Series $\mathrm{B}$, Vol. 61, 51-61.

Rao, B. L. S. P. (1990) Remarks on univariate symmetric distributions. Statistics and Probability Letters, Vol. 10, 307-315.

Seber, G. A. F. e Wild, C. J. (1989) Nolinear Regression. John Wiley.

Sharpe, W. F. (1964). Capital Asset Prices: a Theory of Market Equilibrium Under Conditions of Risk. Journal of Finance, Vol. 19, 425-442.

St. Laurent, R. T. e Cook, R. D. (1992) Leverage and Superleverage in Nonlinear Regression. Journal of the American Statistical Association, Vol. 87, 985-990.

Thejll P. e Schmith T. (2005) Limitations on Regression Analysis Due to Serially Correlated Residuals: Application to Climate Reconstruction from Proxies. Journal of Geophysical Research, Vol. 110, D18103. 
Tsai, C. e Wu X. (1992) Assessing Local Influence in Linear Regression Models with First-Order Autoregressive or Heteroscedastic Error Structure. Statistics \& Probability Letters, Vol. 14, 247-252.

Wei, B. C.; Hu, Y. Q. e Fung, W. K. (1998) Generalized Leverage and Its Applications. Scandinavian Journal of Statistics, Vol. 25, 25-37.

Zind-Walsh, V. e Galbraith J. W. (1991) Estimation of a Linear Regression Model with Stationary ARMA $(p, q)$ Errors. Journal of Econometrics, Vol. 47, 333-357. 